\title{
Conformally invariant energies of knots
}

\author{
R. Langevin and J. O'Hara \\ Laboratoire de Topologie, CNRS-UMR 5584, Université de Bourgogne, \\ Department of Mathematics, Tokyo Metropolitan University
}

October 23, 2018

\begin{abstract}
Conformally invariant functionals on the space of knots are introduced via extrinsic conformal geometry of the knot and integral geometry on the space of spheres. Our functionals are expressed in terms of a complex-valued 2-form which can be considered as the cross-ratio of a pair of infinitesimal segments of the knot. We show that our functionals detect the unknot as the total curvature does, and that their values explode if a knot degenerates to a singular knot with double points.
\end{abstract}

\section{Contents}

\begin{tabular}{llr}
\hline & Introduction. & 2
\end{tabular}

2 The space of spheres and the cross-ratio of 4 points. 4

2.1 The space of $(n-1)$-spheres in $S^{n}$. . . . . . . . . . . . . . . . . . . 4

2.2 The 4 -tuple map and the cross-ratio of 4 points. . . . . . . . . . . . . . . . . 7

$3 \quad$ Review of $r^{-2}$-modified potential energy.

3.1 What is an energy functional for knots? . . . . . . . . . . . . . . . . . 10

3.2 The regularization of $r^{-2}$-modified potential energv $E^{(2)} \ldots \ldots \ldots$

3.3 The cosine formula for $E^{(2)}$. . . . . . . . . . . . . . . . . . . . . . . . 12

4 The infinitesimal cross-ratio. $\quad 14$

4.1 The infinitesimal cross-ratio as a "bilocal" function. . . . . . . . . . . . . . . . . 14

4.2 The 4-tuple map for a knot and the twice tangent spheres. . . . . . . . . . . . . 17

4.3 The real part as the canonical svmplectic form on $T^{*} S^{3}$. . . . . . . . . . . . . . 19

4.3.1 The pull-back of the svmplectic form to $S^{n} \times S^{n} \backslash \triangle$. . . . . . . . . . . 20

4.3.2 From cosine formula to the original definition of $E^{(2)}$. . . . . . . . . . . 24 
5 The (absolute) imaginarv cross-ratio energy, $E_{|\sin \theta|}$. 26

5.1 The projection of the inverted open knot . . . . . . . . . . . . . . 26

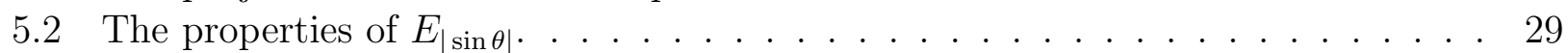

6 Measure of non-trivial spheres.

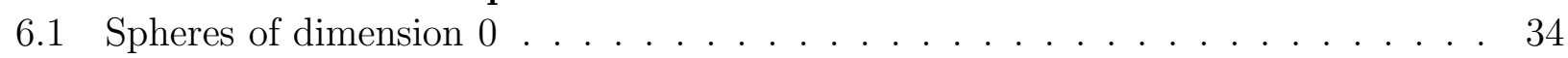

6.2 Non-trivial spheres in $\mathcal{S}$. tangent spheres and twice tangent spheres. . . . . . . 35

6.3 The volume of the set of the non-trivial spheres. . . . . . . . . . . . . . 37

6.4 The measure of acyclicity in terms of the infinitesimal cross-ratio. . . . . . . . 38

$\begin{array}{lll}7 & \text { Non-trivial zones. } & 43\end{array}$

7.1 Non-trivial zones. . . . . . . . . . . . . . . . . . . . . 43

7.1 .1 The modulus of 4 points $\ldots \ldots \ldots$. . . . . . . . . . . 47

7.1 .2 Knots of small moduli . . . . . . . . . . . . . . . . . . . . . . 49

7.2 Jump of the measure of acvclicitv for non-trivial knots. . . . . . . . . . . . . 53

7.3 Explosion of the measure of acyclicity for singular knots. . . . . . . . . . . . 54

8 Appendix. 55

8.1 The maximal modulus and cross-ratio of four points. . . . . . . . . . . 55

8.2 The circular Gauss map and the inverted open knots. . . . . . . . . . . . . . . . 59

8.3 The global radius of curvature. . . . . . . . . . . . . . . . . . . . . . 62

8.4 Links . . . . . . . . . . . . . . . . . . . . . . . . 63

keywords: knot, energy, conformal, cross-ratio, Möbius transformations.

AMS classification: Primary 57M25, 53A30; Secondary 57R17

\section{Introduction.}

In 1929 Fenchel [Fe1 proved that the total curvature of a closed curve is greater than or equal to $2 \pi$. Between 1949 and 1951, Fary [Fa] Fenchel [Fe2] and Milnor [Mi] proved independently that the total curvature of a non-trivial knot is greater than $4 \pi$. This means that non-triviality of knots imposes a jump on the total curvature functional. For knots in $S^{3}$ one has to consider the sum of the length and the total curvature to get such a jump for non-trivial knots ( $\mathrm{Ban}$, La-Ro).

In 1989 the second author introduced the energy $E^{(2)}$ of a knot [O'H1] as the regularization of modified electrostatic energy of charged knots. This energy $E^{(2)}$ is a functional on the space of knots which explodes if a knot degenerates to a singular knot with double points. In 1994 Freedman, He, and Wang [Fr-He-Wa] showed that the energy is conformally invariant and that there is a lower bound for the energy of non-trivial knots, which can detect the unknot.

In this paper we introduce several conformally invariant functionals on the space of knots that satisfy the two properties above, namely, the jump imposed by the non-triviality and the explosion for the singular knots. These functionals can be expressed in terms of a complexvalued 2-form on the configuration space $K \times K \backslash \triangle$ of a knot $K$, which is obtained as the 
cross-ratio of $d x$ and $d y$ on the 2 -sphere $\Sigma \cong \mathbb{C} \cup\{\infty\}$ that is twice tangent to the knot at $x$ and $y$.

This paper is arranged as follows:

In section 2 we introduce the geometry of the space of $n$-spheres. We realize it as a hypersurface in Minkowski space and give an invariant measure on it. A 4-tuple of points of $\mathbb{R}^{3}$ or $S^{3}$ is contained in a sphere $\Sigma$ (unique if the four points do not belong to a circle) which allows us to define the cross-ratio of four points.

In section 3 we make a brief review of the energy $E^{(2)}$, and introduce the cosine formula given by Doyle and Schramm.

In section 4 we show that the integrand of $E^{(2)}$ can be interpreted in terms of the infinitesimal cross-ratio, which is the cross-ratio of four points $(x, x+d x, y, y+d y)$ on the knot, by using the 2-sphere which is twice tangent to the knot at $x$ and $y$. We show that the real part of this infinitesimal cross-ratio coincides with the pull-back of the canonical symplectic form of the cotangent bundle of $S^{3}$, which allows us to deduce the original definition of $E^{(2)}$ from its cosine formula.

In section 5 we define another functional in terms of this infinitesimal cross-ratio and show that it has the jump and the explosion properties.

In section 6 we introduce a non-trivial sphere for a knot, which is a sphere that intersects the knot in at least 4 points. We give a formula of the measure of the set of the non-trivial spheres for a knot (counted with multiplicity), which will be called the measure of acyclicity, in terms of the infinitesimal cross-ratio.

In section 7 we then study the position of the knot with respect to a zone which is a region bounded by two disjoint 2-spheres. Recall that a zone is characterised up to conformal transformation by one number which we call the modulus. One way to compute it is to send the two boundary spheres by a conformal transformation into concentric position. The modulus is then the ratio of the radii. By showing that too "thin" zones can not catch the topology of a given non-trivial knot, we prove that the measure of acyclicity has the jump and the explosion properties.

We put an appendix as section 8. In subsection 8.1 we show that the integrand of $E^{(2)}$ can be interpreted as the maximal modulus of an infinitesimal zone whose boundary spheres pass through pairs of points $\{x, y\}$ and $\{x+d x, y+d y\}$ respectively. In subsection 8.2 we introduce a conformal geometric generalization of the Gauss' integral formula for the linking number. In subsection 8.3 we express the radius of global curvature, which was introduced by Menger and Gonzalez and Maddocks [Go-Ma] in terms of the 4-tuple map introduced in section 2.

Throughout the paper, for simplicity's sake, when we consider knots in $\mathbb{R}^{3}$ we assume that they are parametrized by arc-length unless otherwise mentioned. In what follows a knot $K$ always means the image of an embedding $f$ from $S^{1}$ into $\mathbb{R}^{3}$ or $S^{3}$.

The authors thank J.P. Troalen for making the pictures, and J. Cantarella, Y. Kanda, R. Kusner, J. Maddocks, K. Ono, and D. Rolfsen for useful conversations. 


\section{The space of spheres and the cross-ratio of 4 points.}

\subsection{The space of $(n-1)$-spheres in $S^{n}$.}

Definition 2.1.1 (1) Define an indefinite quadratic form $L$ on $\mathbb{R}^{n+2}$ and the associated pseudo inner product $L(\cdot, \cdot)$

$$
\begin{aligned}
& L\left(x_{1}, \cdots, x_{n+2}\right)=\left(x_{1}\right)^{2}+\cdots+\left(x_{n+1}\right)^{2}-\left(x_{n+2}\right)^{2}, \\
& L(u, v)=u_{1} v_{2}+\cdots u_{n+1} v_{n+1}-u_{n+2} v_{n+2} .
\end{aligned}
$$

This quadratic form with signature $(n+1,1)$ is called the Lorentz quadratic form. The Euclidean space $\mathbb{R}^{n+2}$ equipped with this pseudo inner product $L$ is called the Minkowski space and denoted by $\mathbb{R}^{n+1,1}$.

(2) The set $\mathcal{G}=O(n+1,1)$ of linear isomorphism of $\mathbb{R}^{n+2}$ which preserves this pseudo inner product $L$ is called the (full homogeneous) Lorentz group. We also call it the conformal group for short.

(3) A vector $v$ in $\mathbb{R}^{n+2}$ is called space-like if $L(v)>0$, light-like if $L(v)=0$, and time-like if $L(v)<0$. A line is called space-like (or time-like) if it contains a space-like (or respectively, time-like) vector. The isotropy cone $\left\{v \in \mathbb{R}^{n+2} \mid L(v)=0\right\}$ of $L$ is called the light cone.

(4) The points at infinity of the light cone in the upper half space $\left\{x_{n+2}>0\right\}$ form an $n$-dimensional sphere. Let it be denoted by $S_{\infty}^{n}$. Since it can be considered as the set of lines through the origin in the light cone, it is identified with the intersection $S_{1}^{n}$ of the upper half light cone and the hyperplane $\left\{x_{n+2}=1\right\}$, which is given by $S_{1}^{n}=\left\{\left(x_{1}, \cdots, x_{n+1} \mid\left(x_{1}\right)^{2}+\cdots+\right.\right.$ $\left.\left(x_{n+1}\right)^{2}-1=0\right\}$.

(5) An element of the Lorentz group $\mathcal{G}$ acts on $S_{\infty}^{n}$ as a conformal transformation which is a composition of reflections with respect to $(n-1)$-spheres in $S_{\infty}^{n}$. The set of conformal transformations of $S_{\infty}^{n} \cong \mathbb{R}^{n} \cup\{\infty\}$ is called the conformal group and is also denoted by $\mathcal{G}$.

Claim 2.1.2 Let $\mathcal{S}$ be the set of oriented $(n-1)$-spheres in $S^{n}$, and let $\Lambda=\left\{x \in \mathbb{R}^{n+2} \mid L(x)=\right.$ $1\}$ be the hyperbolic quadric hypersurface of one sheet. (This $\Lambda$ is called de Sitter space.) Then there is a canonical bijection between $\mathcal{S}$ and $\Lambda$.

Proof: A point $x$ in $\Lambda$ determines an oriented half line $l_{x}=\overrightarrow{O x}$ from the origin. Let $\Pi\left(l_{x}\right)$ be the oriented hyperplane passing through the origin that is orthogonal to $l_{x}$ with respect to Lorentz quadratic form $L$. Since $l_{x}$ is space-like $\Pi\left(l_{x}\right)$ intersects the light cone transversely and therefore $\Pi\left(l_{x}\right)$ intersects $S_{\infty}^{3}$ in an oriented sphere $\Sigma_{\Pi\left(l_{x}\right)}$. The map $\Lambda \ni x \mapsto \Sigma_{\Pi\left(l_{x}\right)} \in \mathcal{S}$ defines the bijection from $\Lambda$ to $\mathcal{S}$.

Let us identify $\Lambda$ with $\mathcal{S}$ through this bijection.

Let $S_{1}$ and $S_{2}$ be oriented 2-spheres. Considered as points in $\Lambda, S_{1}$ and $S_{2}$ satisfy:

(1) $S_{1} \cap S_{2} \neq \phi$ if and only if $\left|L\left(S_{1}, S_{2}\right)\right| \leq 1$

(2) $S_{1} \cap S_{2}=\phi$ if and only if $\left|L\left(S_{1}, S_{2}\right)\right|>1$. 


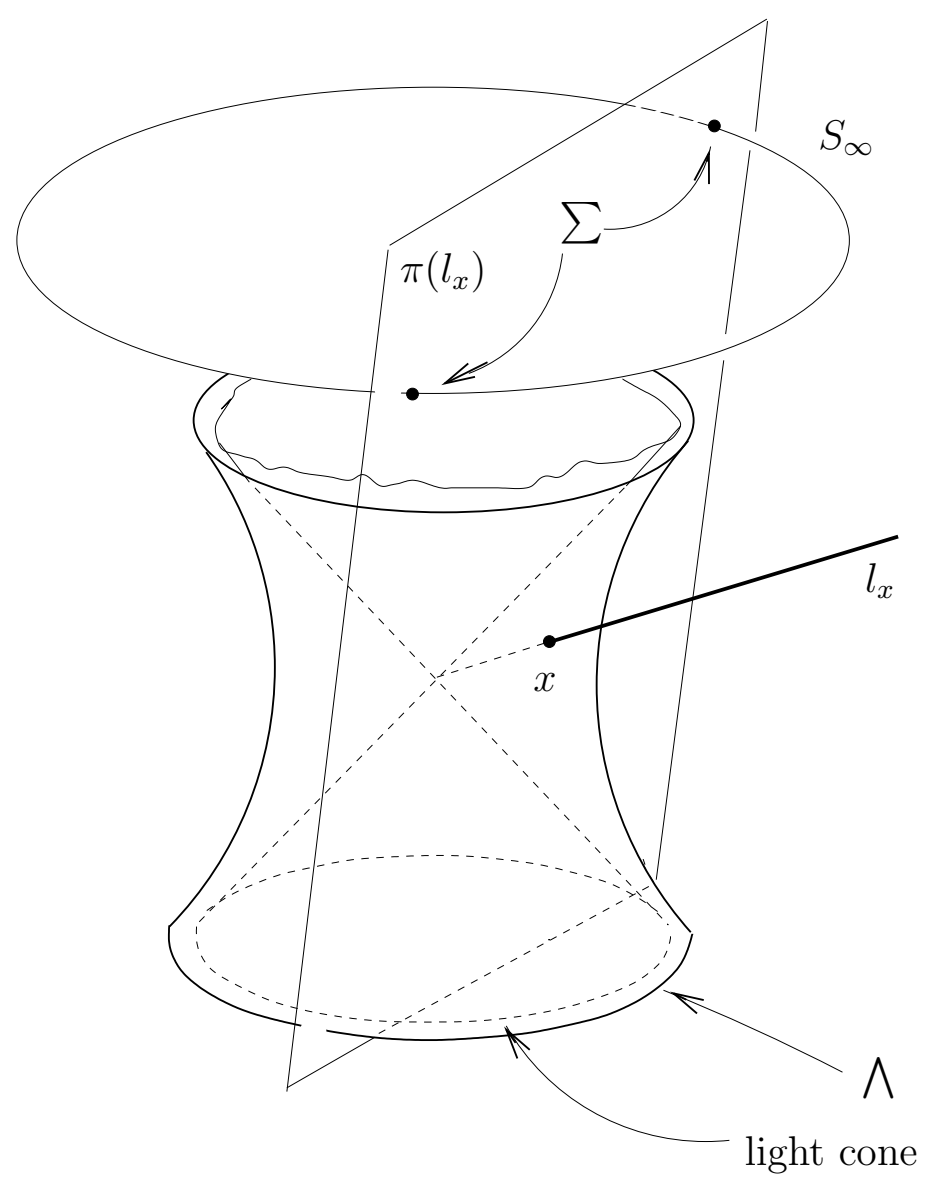

Figure 1: $S_{\infty}^{n}$ and the correspondence between $\Lambda$ and $\mathcal{S}$.

Two spheres $S_{1}$ and $S_{2}$ are said to be nested if $S_{1} \cap S_{2}=\emptyset$ and intersecting if $S_{1} \cap S_{2} \neq \emptyset$ and $S_{1} \neq S_{2}$.

Dimension 2 planes through the origin intersect the quadric $\Lambda \subset \mathbb{R}^{n+2}$ in curves. Those curves are geodesics in the sense that they are critical (but not minimal) points of the length function which assigns the length $\int_{a}^{b} \sqrt{\left|L\left(c^{\prime}(t)\right)\right|} d t$ to any given $\operatorname{arc} c:[a, b] \rightarrow \Lambda$. There are three cases.

(1a) When the plane contains a time-like vector, the intersection consists of two non-compact curves. The pencil of spheres has limit (or Poncelet) points which are $P \cap S_{\infty}^{n}$; it is called a Poncelet pencil.

In case (1) of the formula 1 the length of one of the arcs joining $S_{1}$ to $S_{2}$ in the pencil is the angle $\theta_{0}$ between the two spheres. This angle satisfies: $L\left(S_{1}, S_{2}\right)=\cos \left(\theta_{0}\right)$.

(1b) When the plane is tangent to the light cone, the intersection is again two non-compact curves, but the pencil is made of spheres all tangent at the point $P \cap S_{\infty}^{n}$.

(2) When all the vector of the plane $P$ are space-like, its intersection with $\Lambda$ is connected and closed. Any tangent vector to this intersection is space-like. The corresponding set of spheres is a pencil of spheres with a common $(n-2)$-sphere 
$\Gamma=P^{\perp} \cap S_{\infty}^{n}\left(P^{\perp}\right.$ means the subspace $L-$ orthogonal to $P$, that is $\left.P^{\perp}=\{w \mid L(v, w)=0 \forall v \in P\}\right)$.

In case (2) of the formula 1, we can get an interesting invariant of the annulus bounded by $S_{1}$ and $S_{2}$ from the number $\left|L\left(S_{1}, S_{2}\right)\right|$ (see 7.1): the length $t_{0}$ of the arc of the pencil joining $S_{1}$ to $S_{2}$ satisfies $\left|L\left(S_{1}, S_{2}\right)\right|=C h\left(t_{0}\right)$.
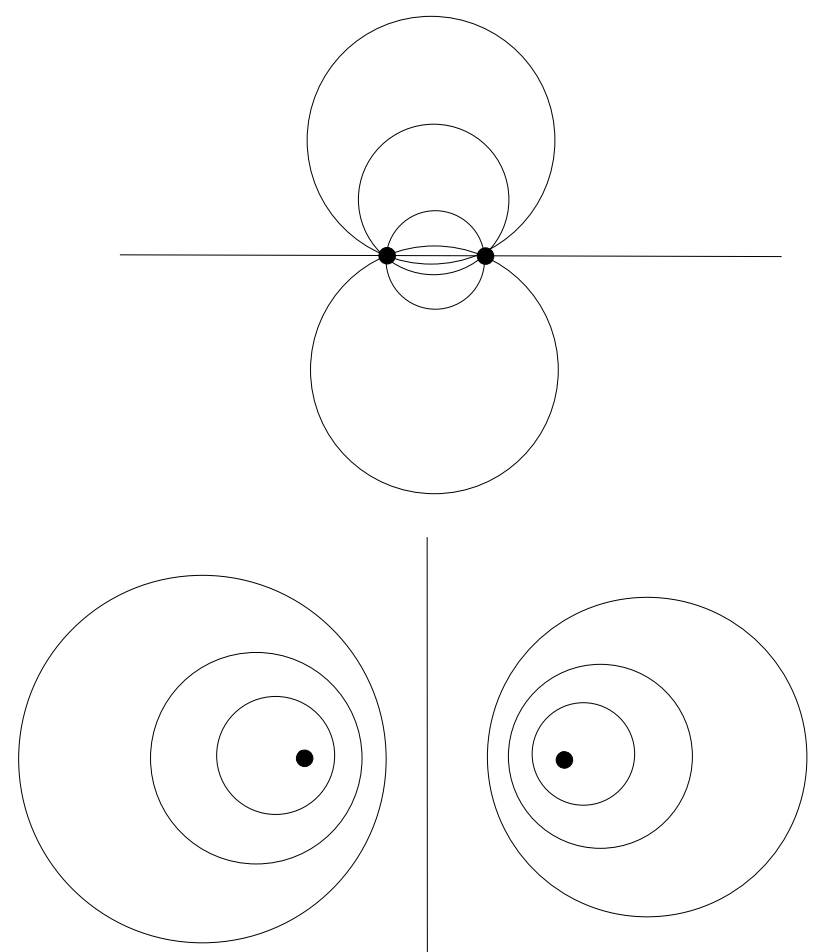

Figure 2: A pencil with a base circle and a Poncelet pencil.

Working in $\Lambda \subset \mathbb{R}^{4}$ we get the "usual" theory of pencils of circles. We will be mainly interested in $\Lambda \subset \mathbb{R}^{5}$ which is the set of oriented 2-spheres in $S^{3}$. We do not study the case when $n>3$.

Remark: Let $\left(v_{1}, v_{2}, \ldots, v_{n+1}\right)$ be $n+1$ vectors in $T_{x} \Lambda$. The volume of the parallelepiped constructed on these vectors is

$$
\left|\operatorname{det}\left(x, v_{1}, v_{2}, \ldots, v_{n+1}\right)\right|=\sqrt{-\operatorname{det}\left(L\left(v_{i}, v_{j}\right)\right)}
$$

This result is a corollary of the lemma 2.2.2,

Let $\Delta=\left\{x \in \mathbb{R}^{n+2} \mid L(x)=-1, x_{n+2}>0\right\}$ be the upper half of the hyperbolic quadric hypersurface of two sheets. The restriction of the quadratic form $L$ to each tangent space of $\Delta$ is positive definite. Then $\Delta$ is a model for the $n+1$-dimensional hyperbolic space $\mathbb{H}=\mathbb{H}^{n+1}$. Each sphere $\Sigma$ in $S^{n}$ is the "boundary at infinity" of a totally geodesic subspace $h$ of $\mathbb{H}$. The 
restriction of the full homogeneous Lorentz group $\mathcal{G}=O(n+1,1)$ to $\mathbb{H}$ is the group of isometries of the hyperbolic space $\mathbb{H}$.

A choice of a point $z$ in $\mathbb{H}$ determines a metric on the sphere $S^{n}$ at infinity of $\mathbb{H}$ by the projection on $S^{n}$ of the metric of $T_{z}(\mathbb{H})$ using the geodesic rays which starts from $z$. Another choice of the point $z$ determines another conformally equivalent metric on the sphere $S^{n}$. Although the sphere does not admit a measure which is invariant under the action of the conformal group $\mathcal{G}$, the set $\mathcal{S} \cong \Lambda$ of spheres in $S^{n}$ do. Namely, $\Lambda$ is endowed with a $\mathcal{G}$-invariant measure $d \Sigma$. We define $d \Sigma$ in several ways in what follows.

(1) The Lebesgue measure $\left|d x_{1} \wedge \cdots \wedge d x_{n+2}\right|$ is invariant under the action of $\mathcal{G}$. It inherits from $\mathbb{R}^{n+2}$ a $\mathcal{G}$-invariant measure defined on each tangent space at a point $x \in \Lambda$ by $d \Sigma=$ $\left|\iota_{x}\left(d x_{1} \wedge \cdots \wedge d x_{n+2}\right)\right|$.

(2) This measure is given by the volume $(n+1)$-form

$$
\omega_{\Lambda}=\frac{\sum_{i=1}^{n+2}(-1)^{i} x_{i} d x_{1} \wedge \cdots \widehat{\wedge d x_{i}} \cdots \wedge d x_{n+2}}{\left(x_{1}^{2}+\cdots+x_{n+1}^{2}-x_{n+2}^{2}\right)^{\frac{n+2}{2}}}
$$

of $\Lambda$ associated with the Lorentz form $L$, where $\widehat{\wedge d x_{i}}$ means that $\wedge d x_{i}$ is removed. Then $\omega_{\Lambda}$ is invariant under the action of $\mathcal{G}_{+}=S O(n+1,1)$. We remark that $\omega_{\Lambda}$ is expressed as

$$
\omega_{\Lambda_{+}}=\frac{1}{x_{n+2}} d x_{1} \wedge \cdots \wedge d x_{n+1}
$$

on $\Lambda_{+}=\left\{\left(x_{1}, \cdots, x_{n+2}\right) \in \Lambda \mid x_{n+2}>0\right\}$ if we take $\left(x_{1}, \cdots, x_{n+1}\right)$ as its local coordinate.

(3) This measure can also be regarded as a measure on the set of totally geodesic hyperplanes of the hyperbolic space $\mathbb{H}$ which is invariant by the action of the hyperbolic isometries [San].

(4) Let us now project the sphere $S^{n}$ stereographically on an affine space $\mathbb{R}^{n}$ with Euclidean coordinates $\left(x_{1}, x_{2}, \ldots, x_{n}\right)$. There, a sphere $\Sigma$ is given by its center $\left(x_{1}, x_{2}, \ldots, x_{n}\right)$ and its radius r. The measure $d \Sigma$ is expressed by

$$
d \Sigma=\frac{1}{r^{n+1}}\left|d x_{1} \wedge d x_{2} \wedge \ldots \wedge d x_{n} \wedge d r\right|
$$

The quadric $\Lambda$ is unbounded and the total measure of $\Lambda$ is infinite. The measure of the set of spheres intersecting a given arc of any size is infinite. Since the set of spheres with geodesical radius larger than $r$ is compact in $\Lambda$, its volume is finite.

Remark: In what follows we assume that circles and spheres include lines and planes respectively as we work in conformal geometry.

\subsection{The 4-tuple map and the cross-ratio of 4 points.}

In this subsection we define the cross-ratio of ordered four points in $S^{3}$ or $\mathbb{R}^{3}$ by means of the oriented 2-sphere that passes through them, which is determined uniquely unless the four points are concircular. 
The configuration space $\operatorname{Conf}_{n}(X)$ of a space $X$ is given by

$$
\begin{aligned}
\operatorname{Conf}_{n}(X) & \left.=\left\{\left(x_{1}, \cdots, x_{n}\right)\right\} \in X^{n} \mid x_{i} \neq x_{j} \text { if } i \neq j\right\} \\
& =X \times \cdots \times X \backslash \triangle
\end{aligned}
$$

where $\triangle$ is called the big diagonal set. Let $\mathcal{C} c\left(S^{3}\right)$ be the subset of $\operatorname{Conf}_{4}\left(S^{3}\right)$ consisting of concircular points.

In what follows we define a map $\Sigma$ from $\operatorname{Conf}_{4}\left(S^{3}\right) \backslash \mathcal{C} c\left(S^{3}\right)$ to the set of oriented 2-spheres, $\mathcal{S} \cong \Lambda$, which assigns to ordered 4 points $(x, y, z, w)$ the oriented 2 -sphere $\Sigma(x, y, z, w)$ that passes through them.

Definition 2.2.1 The (4,1)-exterior product $v^{1} \wedge \cdots \wedge v^{4}$ of ordered four vectors $v^{i}=\left(v_{1}^{i}, \cdots, v_{5}^{i}\right)$ in $\mathbb{R}^{5}(i=1,2,3,4)$ is the uniquely determined vector $u$ that satisfies

$$
L(u, w)=\operatorname{det}\left(v^{1}, v^{2}, v^{3}, v^{4}, w\right) \in \mathbb{R} \quad\left(\forall w \in \mathbb{R}^{5}\right) .
$$

Then it is given by

$$
v^{1} \wedge \cdots \wedge v^{4}=\left(\tilde{\nu}_{1}, \tilde{\nu}_{2}, \tilde{\nu}_{3}, \tilde{\nu}_{4},-\tilde{\nu}_{5}\right)
$$

with

$$
\tilde{\nu}_{j}=(-1)^{j+1}\left|\begin{array}{ccccc}
v_{1}^{1} & \cdots & \hat{v}_{j}^{1} & \cdots & v_{5}^{1} \\
\vdots & & \vdots & & \vdots \\
v_{1}^{4} & \cdots & \hat{v}_{j}^{4} & \cdots & v_{5}^{4}
\end{array}\right|,
$$

where^ means that the $j$-th column is removed.

We remark that this is a generalization of the vector product of two vectors in $\mathbb{R}^{3}$.

Lemma 2.2.2 (1) $v^{1} \wedge v^{2} \wedge v^{3} \wedge v^{4} \neq 0$ if and only if $v^{1}, v^{2}, v^{3}, v^{4}$ are linearly independent.

(2) $L\left(v^{1} \wedge v^{2} \wedge v^{3} \wedge v^{4}, v^{j}\right)=0$ for $j=1,2,3,4$, namely, $v^{1} \wedge v^{2} \wedge v^{3} \wedge v^{4}$ is L-orthogonal to $\operatorname{Span}\left\langle v^{1}, v^{2}, v^{3}, v^{4}\right\rangle$.

(3) The norm of $v^{1} \wedge v^{2} \wedge v^{3} \wedge v^{4}$ is equal to the absolute value of the volume of the parallelepiped spanned by $v^{1}, v^{2}, v^{3}$ and $v^{4}$ associated with the Lorentz quadratic form $L$ :

$$
\sqrt{\left|L\left(v^{1} \wedge v^{2} \wedge v^{3} \wedge v^{4}\right)\right|}=\sqrt{\left|\operatorname{det}\left(L\left(v^{i}, v^{j}\right)\right)\right|} .
$$

Proof: (3) The last equality is a consequence of a formula in linear algebra.

$$
\begin{aligned}
\operatorname{det}\left(L\left(v^{i}, v^{j}\right)\right) & =\operatorname{det}\left\{\left(\begin{array}{ccc}
v_{1}^{1} & \cdots & v_{5}^{1} \\
\vdots & & \vdots \\
v_{1}^{4} & \cdots & v_{5}^{4}
\end{array}\right)\left(\begin{array}{ccc}
v_{1}^{1} & \cdots & v_{1}^{4} \\
\vdots & & \vdots \\
v_{4}^{1} & \cdots & v_{4}^{4} \\
-v_{5}^{1} & \cdots & -v_{5}^{4}
\end{array}\right)\right\} \\
& =-\tilde{\nu}_{1}^{2}-\tilde{\nu}_{2}^{2}-\tilde{\nu}_{3}^{2}-\tilde{\nu}_{4}^{2}+\tilde{\nu}_{5}^{2}
\end{aligned}
$$


Claim 2.2.3 (1) Let $u=\left(u_{1}, \cdots, u_{5}\right)$ and $v=\left(v_{1}, \cdots, v_{5}\right)$ be linearly independent light-like vectors with $u_{5}, v_{5}>0$. Then $L(u, v)<0$.

Therefore, any pair of linearly independent light-like vectors can not be L-orthogonal.

(2) Let $u=\left(u_{1}, \cdots, u_{5}\right)$ be a non-zero light-like vector. Then the hyperplane $u^{\perp}$ is tangent to the light-cone along the generatrix $\mathbb{R} \cdot u$.

(3) If $u=\left(u_{1}, \cdots, u_{5}\right)$ is a non-zero time-like vector, then the hyperplane $u^{\perp}$ is space-like.

Corollary 2.2.4 Suppose $v^{1}, v^{2}, v^{3}, v^{4}$ are linearly independent.

(1) If $v^{1} \wedge v^{2} \wedge v^{3} \wedge v^{4}$ is time-like, then all of $v^{1}, v^{2}, v^{3}, v^{4}$ are space-like.

(2) $v^{1} \wedge v^{2} \wedge v^{3} \wedge v^{4}$ is light-like if and only if $\operatorname{Span}\left\langle v^{1}, v^{2}, v^{3}, v^{4}\right\rangle$ is the tangent space of the light cone at $v^{1} \wedge v^{2} \wedge v^{3} \wedge v^{4}$.

Let us identify the 3-sphere $S^{3}$ with the intersection $S_{1}^{3}$ of the light-cone $\{L=0\}$ and the hyperplane in $\mathbb{R}^{5}$ defined by $\left\{\left(x_{1}, \cdots, x_{5}\right) \mid x_{5}=1\right\}$. Let $x^{i}=\left(x_{1}^{i}, x_{2}^{i}, x_{3}^{i}, x_{4}^{i}\right) \in S^{3}(i=1,2,3,4)$ and $\tilde{x}^{i}=\left(x_{1}^{i}, x_{2}^{i}, x_{3}^{i}, x_{4}^{i}, 1\right) \in S_{1}^{3} \subset \mathbb{R}^{5}$. Then $\tilde{x}^{i}$ 's are linearly dependent if and only if $x^{i}$ 's are concircular.

Since an oriented 2-sphere $\Sigma\left(x^{1}, x^{2}, x^{3}, x^{4}\right)$ that passes through $\left\{x^{1}, x^{2}, x^{3}, x^{4}\right\}$ is obtained as the intersection of $S_{1}^{3}$ and an oriented hyperplane in $\mathbb{R}^{5}$ that passes through $x^{1}, x^{2}, x^{3}, x^{4}$, and the origin, we obtain:

Proposition 2.2.5 Define the "4-tuple map" of the 3-sphere by

$$
\Sigma: \operatorname{Conf}_{4}\left(S^{3}\right) \backslash \mathcal{C} c\left(S^{3}\right) \ni\left(x^{1}, x^{2}, x^{3}, x^{4}\right) \mapsto \frac{\tilde{x}^{1} \wedge \tilde{x}^{2} \wedge \tilde{x}^{3} \wedge \tilde{x}^{4}}{\sqrt{L\left(\tilde{x}^{1} \wedge \tilde{x}^{2} \wedge \tilde{x}^{3} \wedge \tilde{x}^{4}\right)}} \in \Lambda \cong \mathcal{S} .
$$

Then $\Sigma\left(x^{1}, x^{2}, x^{3}, x^{4}\right)$ is the oriented 2-sphere that passes through $\left\{x^{1}, x^{2}, x^{3}, x^{4}\right\}$.

Assume that the complex plane $\mathbb{C}$ has the standard orientation.

Definition 2.2.6 Let $\left(x^{1}, x^{2}, x^{3}, x^{4}\right) \in \operatorname{Conf}_{4}\left(S^{3}\right)$. Let $p: \Sigma\left(x^{1}, x^{2}, x^{3}, x^{4}\right) \rightarrow \mathbb{C}$ be any stereographic projection that preserves the orientation, where, when $x^{1}, x^{2}, x^{3}$, and $x^{4}$ are concircular, we can choose any oriented 2 -sphere that passes through them as $\Sigma\left(x^{1}, x^{2}, x^{3}, x^{4}\right)$.

The cross-ratio of ordered four points $\left(x^{1}, x^{2}, x^{3}, x^{4}\right)$ is defined as the cross-ratio

$$
\left(p\left(\tilde{x}^{2}\right), p\left(\tilde{x}^{3}\right) ; p\left(\tilde{x}^{1}\right), p\left(\tilde{x}^{4}\right)\right)=\frac{p\left(\tilde{x}^{2}\right)-p\left(\tilde{x}^{1}\right)}{p\left(\tilde{x}^{2}\right)-p\left(\tilde{x}^{4}\right)}: \frac{p\left(\tilde{x}^{3}\right)-p\left(\tilde{x}^{1}\right)}{p\left(\tilde{x}^{3}\right)-p\left(\tilde{x}^{4}\right)} .
$$

We denote it by $\left(x^{2}, x^{3} ; x^{1}, x^{4}\right)$. When $x^{1}, x^{2}, x^{3}$ and $x^{4}$ are concircular, their cross-ratio is real and independent of the choice of $\Sigma\left(x^{1}, x^{2}, x^{3}, x^{4}\right)$.

Lemma 2.2.7 The image of $\operatorname{Conf}_{4}\left(S^{3}\right) \backslash \mathcal{C} c\left(S^{3}\right)$ by the cross-ratio map of formula 2 is contained in a component of $\mathbb{C} \backslash \mathbb{R}$.

Proof: The set $\triangle \subset\left(S^{3}\right)^{4}$ is of codimension 3 ; the set $\mathcal{C} c\left(S^{3}\right)$ is of codimension 2 in Conf $_{4}\left(S^{3}\right)$ and the cross-ratio map is continuous on the arcwise connected set $\operatorname{Conf}_{4}\left(S^{3}\right) \backslash \mathcal{C} c\left(S^{3}\right)$.

The 4-tuple map $\Sigma$ of $\mathbb{R}^{3}$ and the cross-ratio of four points in $\mathbb{R}^{3}$ are defined through an orientation preserving stereographic projection : $p: S^{3} \rightarrow \mathbb{R}^{3}$. When $x^{1}, x^{2}, x^{3}$, and $x^{4}$ are concircular in $\mathbb{R}^{3}$, we define $\Sigma\left(x^{1}, x^{2}, x^{3}, x^{4}\right)$ to be any oriented sphere that passes through them. 


\section{Review of $r^{-2}$-modified potential energy.}

\subsection{What is an energy functional for knots?}

In the following three sections we study knots in $\mathbb{R}^{3}$. By an open knot we mean an embedding $\tilde{f}$ from a line into $\mathbb{R}^{3}$ or its image $\tilde{K}=\tilde{f}(\mathbb{R})$ that approaches asymptotically a straight line at both ends. We assume that knots are of class $C^{2}$.

The energy of knots was proposed by Fukuhara [Fu and Sakuma $[\mathrm{Sak}$ motivated by the following problem: Define a suitable functional on the space of knots, which we call "energy", and define a good-looking "canonical knot" for each isotopy class as one of the embeddings that attain the minimum value of this "energy" within its isotopy class.

For this purpose we try to deform a given embedding along the negative gradient flow of the "energy" until it comes to a critical point without changing its knot type. Hence the crossing changes should be avoided.

Thus we are lead to the notion of the energy functional for knots which is a functional that explodes if a knot degenerates to an immersion with double points. More precisely;

Definition 3.1.1 A functional $e$ on the space of knots is an energy functional for knots if for any real numbers $b$ and $\delta$ with $0<\delta \leq \frac{1}{2}$, there exists a positive constant $C=C(b, \delta)$ which satisfies the following condition:

If a knot $f$ with length $l(f)$ contains a pair of points $x$ and $y$ which satisfy that the shorter arc-length between them is equal to $\delta l(f)$ and that $|x-y| \leq C l(f)$, then $e(f) \geq b$.

We call a functional an energy functional in the weak sense when it satisfies the same condition as above if the knot $f$ satisfies an additional geometric condition; for example, if the curvature of $f$ is bounded above by a constant.

\subsection{The regularization of $r^{-2}$-modified potential energy, $E^{(2)}$.}

One of the most natural and naïve candidates for an energy functional for knots would be the electrostatic energy of charged knots.

The first attempt to consider the electrostatic energy of charged knots was carried out in the finite dimensional category by Fukuhara $[\mathrm{Fu}$. He considered the space of the polygonal knots whose vertices are charged, and studied their modified electrostatic energy under the assumption that Coulomb's repelling force between a pair of point charges of distance $r$ is proportional to $r^{-m}(m=3,4,5, \cdots)$.

The first example of an energy functional for smooth knots was defined by the second author in $\mathrm{O}^{\prime} \mathrm{H} 1$. (The reader is referred to a survey article ['H2 for details.)

Let $K=f\left(S^{1}\right)$ with $f: S^{1}=[0,1] / \sim \rightarrow \mathbb{R}^{3}$ be a knot of class $C^{2}$ that is parametrized by the arc-length. Suppose that the knot is uniformly electrically charged. In order to obtain an energy functional for knots, we have to make the non-realistic assumption that Coulomb's repelling force between a pair of point charges of distance $r$ is proportional to $r^{-(\alpha+1)}$ and 
hence the potential is proportional to $r^{-\alpha}$ where $\alpha \geq 2$. Put $\alpha=2$ in what follows. Then its " $r^{-2}$-modified potential energy" is given by

$$
\iint_{K \times K} \frac{d x d y}{|x-y|^{2}}
$$

which blows up at the diagonal set $\triangle$ for any knot. We normalize this blowing up in the following way:

Let $\delta_{K}(x, y)$ denote the shorter arc-length between $x$ and $y$ along the knot $K$. Define the " $r^{-2}$-modified $\epsilon$-self avoiding voltage" at a point $x, V_{\epsilon}^{(2)}(K ; x)$, and the " $r^{-2}$-modified $\epsilon$-offdiagonal potential energy" $E_{\epsilon}^{(2)}(K)$ by

$$
\begin{aligned}
V_{\epsilon}^{(2)}(K ; x) & =\int_{y \in K, \delta_{K}(x, y) \geq \epsilon} \frac{d y}{|x-y|^{2}} \\
E_{\epsilon}^{(2)}(K) & =\int_{K} V_{\epsilon}^{(2)}(K ; x) d x=\iint_{K \times K, \delta_{K}(x, y) \geq \epsilon} \frac{d x d y}{|x-y|^{2}}
\end{aligned}
$$

Then the order of the blowing up of $V_{\epsilon}^{(2)}(K ; x)$ and $E_{\epsilon}^{(2)}(K)$, as $\epsilon$ goes down to 0 , is independent of the knot $K$ and the point $x$. Therefore we have the limits:

$$
\begin{aligned}
V^{(2)}(K ; x) & =\lim _{\epsilon \rightarrow 0}\left(\int_{y \in K, \delta_{K}(x, y) \geq \epsilon} \frac{d y}{|x-y|^{2}}-\frac{2}{\epsilon}\right)+4, \\
E^{(2)}(K) & =\lim _{\epsilon \rightarrow 0}\left(\iint_{K \times K, \delta_{K}(x, y) \geq \epsilon} \frac{d x d y}{|x-y|^{2}}-\frac{2}{\epsilon}\right)+4 .
\end{aligned}
$$

Then $E^{(2)}(K)$ is equal to

$$
E^{(2)}(K)=\iint_{K \times K}\left(\frac{1}{|x-y|^{2}}-\frac{1}{\delta_{K}(x, y)^{2}}\right) d x d y .
$$

We remark that the integrand converges near the diagonal set for knots of class $C^{4}$. This expression ${ }^{1}$ works for open knots and (open) knots which are not necessarily parametrized by arc-length.

Then $E^{(2)}$ is an energy functional for knots. In fact we can take a constant $C(b)$ which is independent of $\delta$ as $C(b, \delta)$ in Definition 3.1.1.

Freedman, He and Wang showed that $E^{(2)}$ is conformally invariant in [Fr-He-Wa]. After their proof, Doyle and Schramm obtained a new formula for $E^{(2)}$ from a geometric interpretation, which implies a simpler proof. We introduce their formula in the next subsection.

\footnotetext{
${ }^{1}$ The idea of using the arc-length as the subtracting counter term to cancel the blowing up of the integral at the diagonal set was introduced by Nakauchi $\mathrm{Na}$.
} 
Remark: If the power " 2 " in the formula of $E^{(2)}$ is replaced by $\alpha$ with $\alpha \geq 1$, then $E^{(\alpha)}$ can be defined similarly if $\alpha<3$. $E^{(\alpha)}$ thus defined is an energy functional for knots if and only if $\alpha \geq 2$ and is conformally invariant if and only if $\alpha=2$.

The jump of $E^{(2)}$ for non-trivial knots is shown as follows.

Freedman and He defined the average crossing number ac $(K)$ of a knot as the average of the numbers of the crossing points of the projected knot diagrams, where the average is taken over all the directions of the projection ( $\mathrm{Fr}-\mathrm{He}]$ ).

Then Freedman, He, and Wang showed that $E^{(2)}$ bounds the average crossing number from above ([Fr-He-Wa $]$, which implies the infimum of the value of $E^{(2)}$ for non-trivial knots, because the average crossing number of a non-trivial knot is greater than or equal to 3 .

\subsection{The cosine formula for $E^{(2)}$.}

In this subsection we introduce the cosine formula for $E^{(2)}$ invented by Doyle and Schramm (see $\mathrm{Au}-\mathrm{Sa}$ ).

Let us first recall a couple of formulae in the conformal geometry. Let $T$ be a conformal transformation of $\mathbb{R}^{3} \cup\{\infty\}$. Put

$$
\left|T^{\prime}(p)\right|=|\operatorname{det} d T(p)|^{\frac{1}{6}}
$$

for $P \in \mathbb{R}^{3}$. In particular, if $T$ is a reflection with respect to a sphere of radius $r$ with center 0 then $\left|T^{\prime}(p)\right|=\frac{r}{|p|}$. Then

$$
|T(p)-T(q)|=\left|T^{\prime}(p)\right|\left|T^{\prime}(q)\right||p-q|
$$

for $p, q \in \mathbb{R}^{3}$ and

$$
\left|(T \circ f)^{\prime}(s)\right|=\left|T^{\prime}(f(s))\right|^{2}\left|f^{\prime}(s)\right|
$$

for $f: S^{1}$ or $\mathbb{R} \rightarrow \mathbb{R}^{3}$.

Let $I_{x}$ be the reflection with respect to the sphere with center $x$ and radius $1, \tilde{K}_{x}=I_{x}(K)$, $\tilde{y}=I_{x}(y), p_{ \pm}(\epsilon)=f(s \pm \epsilon)$, and $\tilde{p}_{ \pm}(\epsilon)=I_{x}\left(p_{ \pm}(\epsilon)\right)$ for $0<\epsilon \ll 1$. We call $\tilde{K}_{x}$ the inverted open knot of $K$ at $x$.

Then

$$
\left|I_{x}^{\prime}(y)\right|=\frac{1}{|x-y|}
$$

and

$$
|d \tilde{y}|=\left|d\left(I_{x}(y)\right)\right|=\left|I_{x}^{\prime}(y)\right|^{2}|d y|=\frac{|d y|}{|x-y|^{2}} .
$$

For us $d x$ and $d y$ are infinitesimal arcs on $K \subset \mathbb{R}^{3}$ or $S^{3}$, and $|d x|,|d y|$, their lengths. We may, later, when the context is clear, use also $d x$ and $d y$ as two one-forms on $K \times K \backslash \Delta$.

Therefore

$$
V_{\epsilon}^{(2)}(K ; x)=\int_{y \in K, \delta(x, y) \geq \epsilon} \frac{d y}{|x-y|^{2}}=\int_{\tilde{p}_{+}(\epsilon)}^{\tilde{p}_{-}(\epsilon)}|d \tilde{y}|
$$

is the arc-length between $\tilde{p}_{+}(\epsilon)$ and $\tilde{p}_{-}(\epsilon)$ along the inverted knot $\tilde{K}_{x}$. 

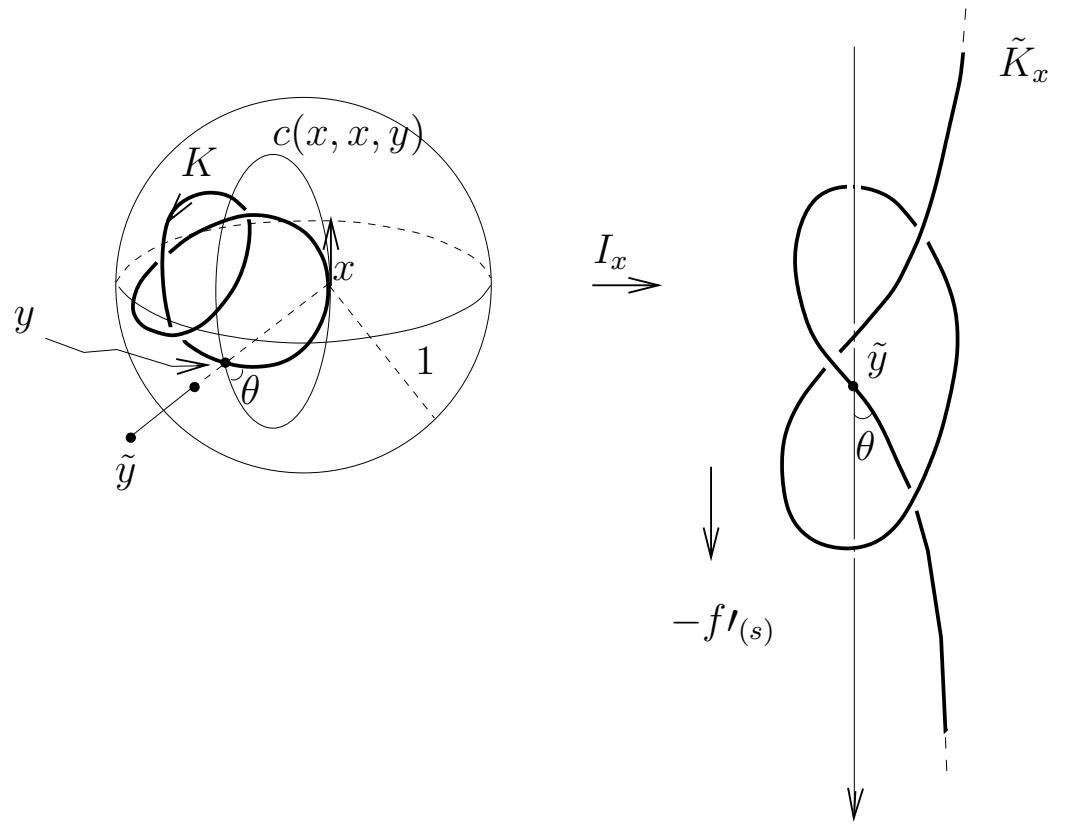

Figure 3: The inverted open knot $\tilde{K}_{x}$ and the angle $\theta$.

Let $\bar{t}=f^{\prime}(s)$ be the unit tangent vector, $n=f^{\prime \prime}(s) /\left|f^{\prime \prime}(s)\right|$ be the unit principal normal vector, and $k=\left|f^{\prime \prime}(s)\right|$ be the curvature of $K$ at $x$. Put $n=0$ when $\left|f^{\prime \prime}(s)\right|=0$. Then

$$
p_{ \pm}(\epsilon)=x \pm \epsilon \bar{t}+\frac{\epsilon^{2} k}{2} n+O\left(\epsilon^{3}\right)
$$

which implies

$$
\tilde{p}_{ \pm}(\epsilon)=x \pm \frac{1}{\epsilon} \bar{t}+\frac{k}{2} n+O(\epsilon) .
$$

Hence

$$
\left|\tilde{p}_{+}(\epsilon)-\tilde{p}_{-}(\epsilon)\right|=\frac{2}{\epsilon}+O(\epsilon)
$$

Therefore

$$
\int_{y \in K, \delta(x, y) \geq \epsilon} \frac{d y}{|x-y|^{2}}-\frac{2}{\epsilon}=\int_{\tilde{p}_{+}(\epsilon)}^{\tilde{p}_{-}(\epsilon)}|d \tilde{y}|-\left|\tilde{p}_{+}(\epsilon)-\tilde{p}_{-}(\epsilon)\right|+O(\epsilon)
$$

is equal to the difference of the arc-length along $\tilde{K}_{x}$ and the distance between $\tilde{p}_{+}(\epsilon)$ and $\tilde{p}_{-}(\epsilon)$ up to $O(\epsilon)$. (This was called the "wasted length" of the inverted open knot.)

Definition 3.3.1 Let $C(x, x, y)$ denote the circle ${ }^{2}$ tangent to a knot $K$ at $x$ that passes through $y$ with the natural orientation derived from that of $K$ at point $x$, and let $\theta(0 \leq \theta \leq \pi)$ be the angle between $C(x, x, y)$ and $C(y, y, x)$ at $x$ or at $y$.

\footnotetext{
${ }^{2}$ In general $C(x, y, z)$ will denote the uniquely determined circle that passes through $x, y$ and $z$ after the notation of Go-Ma.
} 
We call $\theta=\theta_{K}(x, y)$ the conformal angle.

Put $\tilde{C}(\infty, \infty, \tilde{y})=I_{x}(C(x, x, y))$. Then $\tilde{C}(\infty, \infty, \tilde{y})$ is the line which passes through $\tilde{y}$ and has the tangent vector $-f^{\prime}(s)$. The line passing through $\tilde{p}_{+}(\epsilon)$ and $\tilde{p}_{-}(\epsilon)$ approaches parallel as $\epsilon$ goes down to 0 to the line $\tilde{C}(\infty, \infty, \tilde{y})$. Since $I_{x}$ is a conformal map the angle between the line $\tilde{C}(\infty, \infty, \tilde{y})$ and the tangent line of $\tilde{K}_{x}$ at $\tilde{y}$ is equal to $\theta$. Therefore

$$
\begin{aligned}
V^{(2)}(K ; x)-4 & =\lim _{\epsilon \rightarrow 0}\left(\int_{y \in K, \delta(x, y) \geq \epsilon} \frac{d y}{|x-y|^{2}}-\frac{2}{\epsilon}\right) \\
& =\lim _{\epsilon \rightarrow 0} \int_{\tilde{p}_{+}(\epsilon)}^{\tilde{p}_{-}(\epsilon)} d \tilde{y}(1-\cos \theta)=\lim _{\epsilon \rightarrow 0} \int_{y \in K} \frac{(1-\cos \theta) d y}{|x-y|^{2}} .
\end{aligned}
$$

Hence

$$
E^{(2)}(K)=\iint_{K \times K} \frac{1-\cos \theta}{|x-y|^{2}} d x d y+4,
$$

which is called the cosine formula for $E^{(2)}$ by Doyle and Schramm.

\section{The infinitesimal cross-ratio.}

In this section we introduce an infinitesimal interpretation of the 2-form

$$
\frac{d x d y}{|x-y|^{2}}
$$

in the integrand of $E^{(2)}$ in terms of the cross-ratio.

\subsection{The infinitesimal cross-ratio as a "bilocal" function.}

In what follows in this section, we use the following notation:

$$
K=f\left(S^{1}\right), x=f(s), x+d x=f(s+d s), y=f(t), \text { and } y+d y=f(t+d t) .
$$

As is stated in subsection 2.2, we can define the cross-ratio $(x+d x, y ; x, y+d y)$ of the ordered four points $x, x+d x, y, y+d y$ via the oriented 2 -sphere $\Sigma(x, x+d x, y, y+d y)$ that passes through them which is uniquely defined unless the four points are concircular.

Definition 4.1.1 A twice tangent sphere $\Sigma_{K}(x, y)$ is an oriented 2-sphere that is tangent to the knot $K$ at the points $x$ and $y$ obtained as the limit of the sphere $\Sigma(x, x+d x, y, y+d y)$ as $d x$ and $d y$ go to 0 .

It is uniquely determined unless $f(s), f^{\prime}(s), f(t)$, and $f^{\prime}(t)$ are concircular. We will give a formula for the twice tangent spheres in the next subsection.

Let $\Pi=\Pi_{x}(y)$ be the plane which contains $C(x, x, y)$ with an identification with the complex plane $\mathbb{C}$, and let

$$
S: \Sigma_{K}(x, y) \rightarrow \Pi_{x}(y) \cup\{\infty\} \cong \mathbb{C} \cup\{\infty\}
$$




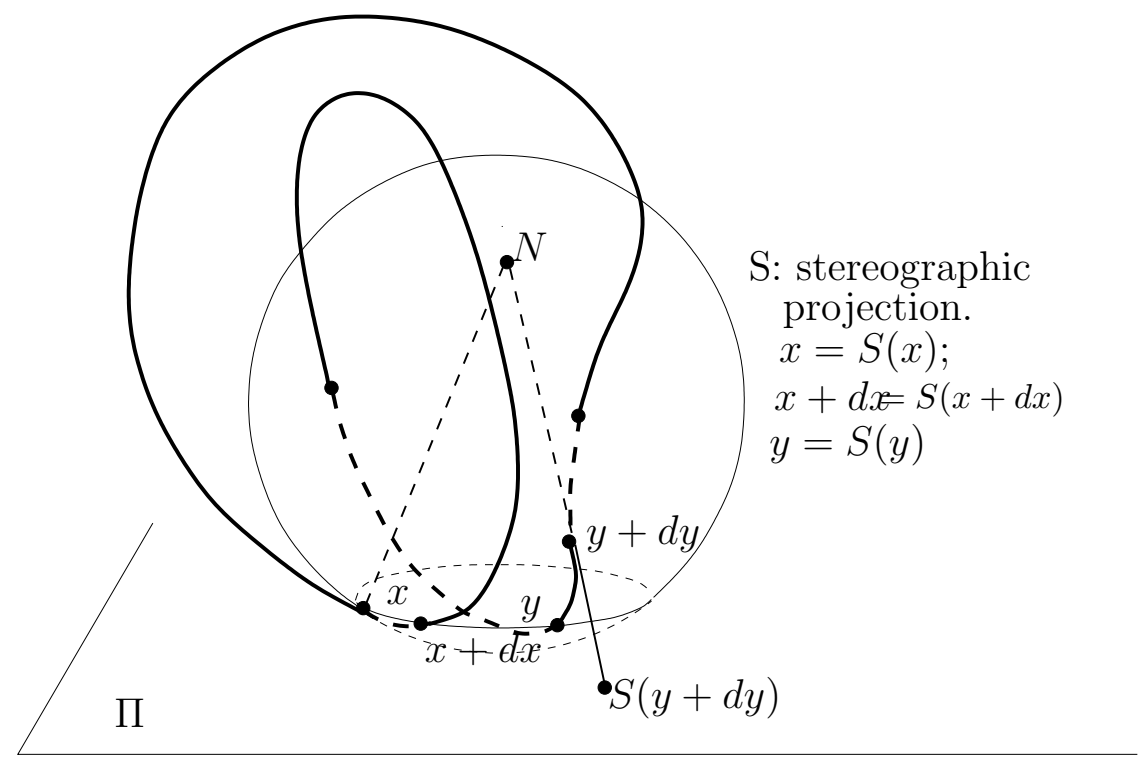

Figure 4: The twice tangent sphere and the infinitesimal cross-ratio.

be the orientation preserving stereographic projection. When $f(s), f^{\prime}(s), f(t)$, and $f^{\prime}(t)$ are concircular, we can take $\Pi$ with any orientation as $\Sigma_{K}$. Let $\tilde{x}=S(x), \tilde{x}+\widetilde{d x}=S(x+d x)$, $\tilde{y}=S(y)$, and $\tilde{y}+\widetilde{d y}=S(y+d y)$ denote the corresponding complex numbers. Then the cross ratio $(x+d x, y ; x, y+d y)$ is given $b^{3}$

$$
\frac{(\tilde{x}+\widetilde{d x})-\tilde{x}}{(\tilde{x}+\widetilde{d x})-(\tilde{y}+\widetilde{d y})}: \frac{\tilde{y}-\tilde{x}}{\tilde{y}-(\tilde{y}+\widetilde{d y})} \sim \frac{\widetilde{d x} \widetilde{d y}}{(\tilde{x}-\tilde{y})^{2}}
$$

Let $v_{x}(y)$ be the unit tangent vector of $C(x, x, y)$ at $y$.

Definition 4.1.2 We call $v_{x}(y)$ the unit tangent vector at $x$ conformally translated to $y$.

Let $\theta_{K}(x, y)\left(-\pi \leq \theta_{K}(x, y) \leq \pi\right)$ be the angle from $v_{x}(y)$ to $f^{\prime}(t)$, where the sign of $\theta_{K}(x, y)$ is given according to the orientation of $\Sigma_{K}(x, y)$. Then $\left|\theta_{K}(x, y)\right|$ is equal to the angle $\theta$ between $C(x, x, y)$ and $C(y, y, x)$ in the cosine formula. As $x, x+d x$ and $y$ are in $\Sigma_{K}(x, y) \cap \Pi$, they are invariant by $p$.

Since $|\widetilde{d y}|=|d y|$ as illustrated on the Figures 4.1, which consider the case when the argument of the cross-ratio is negative, the cross-ratio, $(x+d x, y ; x, y+d y)$ has the absolute value :

$$
\frac{|d x||d y|}{|x-y|^{2}}
$$

and the argument: $\theta_{K}(x, y)$.

\footnotetext{
${ }^{3}$ R. Kusner already mentionned such an infinitesimal cross-ratio in joint AMS-SMM meeting in Oaxaca, Mexico in the fall of 1997 and in a talk on quadrupoles in Illinois (1998).
} 

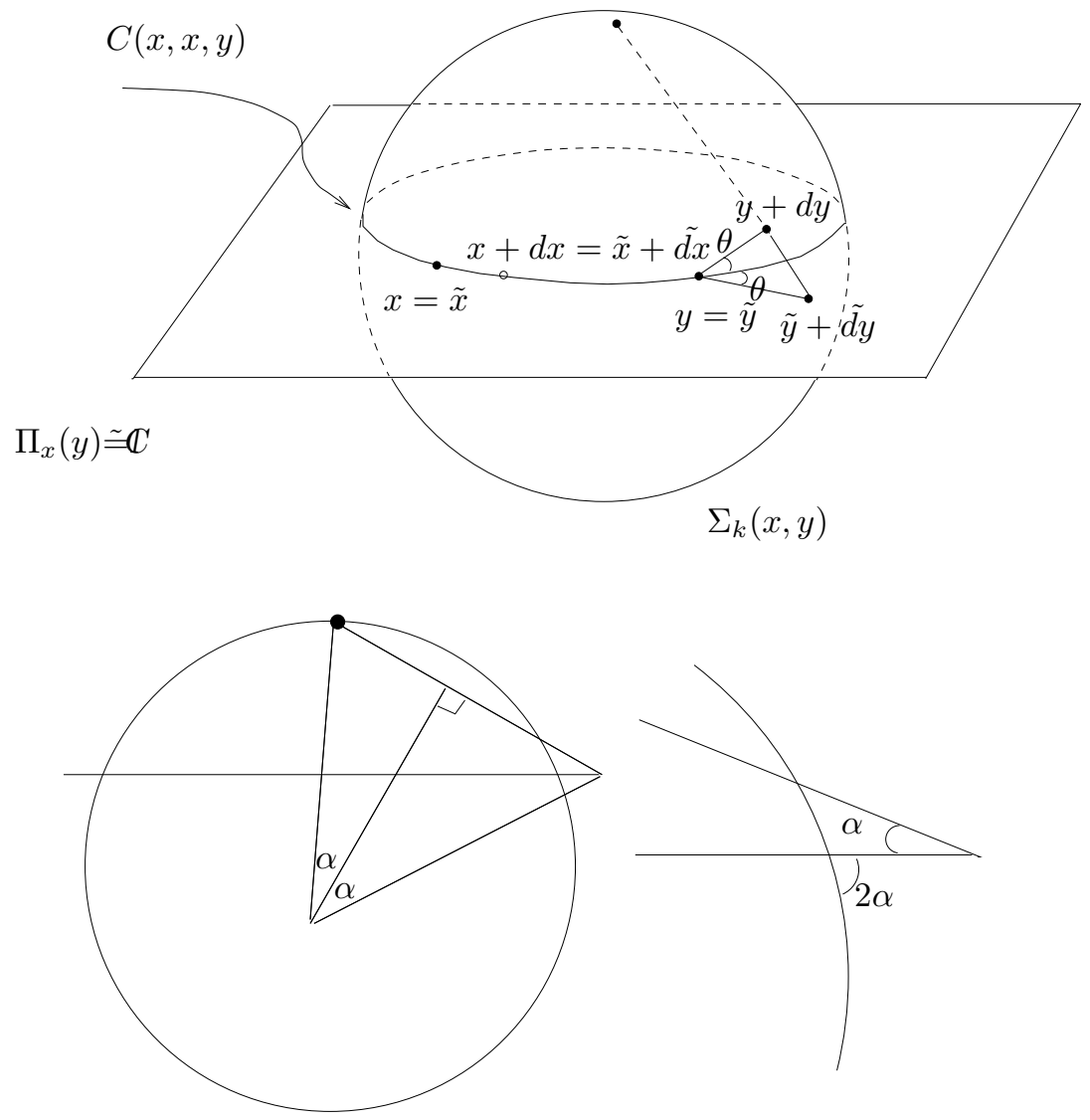

Figure 5: The twice tangent sphere, stereographic projection and computation of the infinitesimal cross-ratio.

Definition 4.1.3 We call $(x+d x, y ; x, y+d y)$ the infinitesimal cross-ratio of $d x$ and $d y$ and denote it by $\Omega_{C R}$.

A stereographic projection from a sphere is a restriction of a conformal transformation of $\mathbb{R}^{3} \cup\{\infty\}$. Hence the infinitesimal cross-ratio (or its is complex conjugate class) is the unique invariant of $d x$ and $d y$ under the action of the orientation preserving conformal group $\mathcal{G}_{+}$(or respectively, the action of the conformal group $\mathcal{G}$ ).

Since the absolute value of the 2 -form

$$
\frac{|d x||d y|}{|x-y|^{2}}
$$

on $K \times K \backslash \triangle$ is the absolute value of $\Omega_{C R}$ and the angle $\theta(0 \leq \theta \leq \pi)$ is the absolute value of the argument of $\Omega_{C R}$, the cosine formula (4) implies

$$
E^{(2)}(K)=\iint_{K \times K \backslash \triangle}\left\{\left|\Omega_{C R}\right|-\Re e \Omega_{C R}\right\}+4 .
$$

Therefore it is conformally invariant.

We close this subsection with the following claim which we will use later. 
Claim 4.1.4 The argument of cross-ratio of a knot $K$ is identically 0 if and only if $K$ is the standard circle.

Proof: Fix a point $x \in K$ and consider the inverted open knot at $x, \tilde{K}_{x}$. Since $\theta(x, y)=0$ for any $y \in K$, the tangent vector of $\tilde{K}_{x}$ at $\tilde{y}$ is equal to $-f^{\prime}(s)$ for any $\tilde{y} \in \tilde{K}_{x}$. Therefore $\tilde{K}_{x}$ is the straight line, which means that $K$ is the standard circle.

\subsection{The 4-tuple map for a knot and the twice tangent spheres.}

In this subsection we define a map that assigns the oriented 2-sphere that passes through a given 4-tuples of points on a knot, and then, a map that assigns the twice tangent sphere.

Definition 4.2.1 The open concircular point set $\mathcal{C} c(K)$ of a knot $K$ is given by $\mathcal{C} c(K)=$ $\left(f^{4}\right)^{-1}\left(\mathcal{C} c\left(S^{3}\right)\right)$, where $f^{4}=f \times f \times f \times f: \operatorname{Conf}_{4}\left(S^{1}\right) \rightarrow \operatorname{Conf}_{4}\left(S^{3}\right)$ is the natural map.

Suppose $K$ is a non-trivial knot. G. Kuperberg showed that $K$ contains 4 points which are collinear $([\mathrm{Ku}])$. As a corollary, $\mathcal{C} c(K)$ is not empty.

Question 4.2.2 Is $\mathcal{C} c(K)$ not empty for any trivial knot $K$ ?

Moreover we will show later that $\mathcal{C} c(K)$ is generically of dimension 2 . This is indicated by the following observation:

Let $K_{p}=I_{\Sigma_{p}}(K)$ be the inverted closed knot with respect to a sphere with center $p \in \mathbb{R}^{3} \backslash K$. Then G. Kuperberg's theorem implies that there is a line $L_{p}$ satisfying that $L_{p} \cap K_{p}$, and hence $I_{p}\left(L_{p}\right) \cap K$, contains 4 points. This means that for any point $p \in \mathbb{R}^{3} \backslash K$ there is a circle $C_{p}$ that intersects $K$ in at least 4 points. This implies that, by considering the normal plane to $C_{p}$ at $p$, the set of such circles, and hence $\mathcal{C} c(K)$ has dimension 2 .

Assume that

$$
f\left(t_{i}\right)=\left(f_{1}\left(t_{i}\right), f_{2}\left(t_{i}\right), f_{3}\left(t_{i}\right), f_{4}\left(t_{i}\right), 1\right) \in S_{1}^{3}=\{L=0\} \cap\left\{x_{5}=1\right\} \subset \mathbb{R}^{5}
$$

for $i=1,2,3,4$. Put

$$
\nu_{f}=\nu_{f}\left(t_{1}, t_{2}, t_{3}, t_{4}\right)=\left(\nu_{1}, \cdots, \nu_{5}\right)=f\left(t_{1}\right) \wedge f\left(t_{2}\right) \wedge f\left(t_{3}\right) \wedge f\left(t_{4}\right) .
$$

We remark that $\nu_{5}\left(t_{1}, t_{2}, t_{3} t_{4}\right)=0$ if and oly if $f\left(t_{i}\right)$ 's are linearly dependent as vectors in $\mathbb{R}^{5}$, which means that $f\left(t_{i}\right)$ 's are concircular as vectors in $S_{1}^{3} \cong S^{3}$.

Definition 4.2.3 The 4-tuple map $\psi_{K}$ for a knot $K$ is a map which assigns the oriented 2sphere that passes through $f\left(t_{i}\right)(i=1,2,3,4)$ to a 4 -tuple $\left(t_{1}, t_{2}, t_{3}, t_{4}\right) \in \operatorname{Conf}_{4}\left(S^{1}\right) \backslash \mathcal{C} c(K)$, which is given by

$$
\begin{aligned}
\psi_{K}=\Sigma \circ f^{4}: \operatorname{Conf}_{4}\left(S^{1}\right) \backslash \mathcal{C} c(K) \ni\left(t_{1}, t_{2}, t_{3}, t_{4}\right) \mapsto & \mapsto f\left(t_{1}\right) \wedge f\left(t_{2}\right) \wedge f\left(t_{3}\right) \wedge f\left(t_{4}\right) \\
& \frac{\nu_{f}}{\sqrt{L\left(\nu_{f}\right)}}=\frac{\left.f\left(t_{3}\right) \wedge f\left(t_{4}\right)\right)}{\sqrt{L\left(f\left(t_{1}\right) \wedge f\left(t_{2}\right) \wedge f\left(t_{3}\right)\right.}} \in \Lambda \cong \mathcal{S} .
\end{aligned}
$$


Claim 4.2.4 The open concircular point set $\mathcal{C} c(K)$ is of dimension more than or equal to 2 if it is not an empty set, especially if $K$ is a non-trivial knot.

Proof: Assume that $\left(t_{1}, t_{2}, t_{3}, t_{4}\right) \in \mathcal{C} c(K) \in$ Conf $_{4}$. Then, considered as vectors in $\mathbb{R}^{5}$, $f\left(t_{1}\right), f\left(t_{2}\right), f\left(t_{3}\right)$, and $f\left(t_{4}\right)$ are linearly dependent, but three of them, say, $f\left(t_{1}\right), f\left(t_{2}\right)$, and $f\left(t_{3}\right)$ are linearly independent. Suppose

$$
f\left(t_{4}\right)=a f\left(t_{1}\right)+b f\left(t_{2}\right)+c f\left(t_{3}\right)
$$

Then

$$
\begin{aligned}
& \frac{\partial \nu_{f}}{\partial t_{1}}=-a f\left(t_{1}\right) \wedge f\left(t_{2}\right) \wedge f\left(t_{3}\right) \wedge f^{\prime}\left(t_{1}\right) \\
& \frac{\partial \nu_{f}}{\partial t_{2}}=-b f\left(t_{1}\right) \wedge f\left(t_{2}\right) \wedge f\left(t_{3}\right) \wedge f^{\prime}\left(t_{2}\right) \\
& \frac{\partial \nu_{f}}{\partial t_{3}}=-c f\left(t_{1}\right) \wedge f\left(t_{2}\right) \wedge f\left(t_{3}\right) \wedge f^{\prime}\left(t_{3}\right) \\
& \frac{\partial \nu_{f}}{\partial t_{4}}=f\left(t_{1}\right) \wedge f\left(t_{2}\right) \wedge f\left(t_{3}\right) \wedge f^{\prime}\left(t_{4}\right) .
\end{aligned}
$$

Since $f\left(t_{1}\right), f\left(t_{2}\right)$, and $f\left(t_{3}\right)$ are linearly independent,

$$
\operatorname{Span}\left\langle\frac{\partial \nu_{f}}{\partial t_{1}}, \frac{\partial \nu_{f}}{\partial t_{2}}, \frac{\partial \nu_{f}}{\partial t_{3}}, \frac{\partial \nu_{f}}{\partial t_{4}}\right\rangle
$$

has dimension at most 2. Therefore the kernel of $d \nu_{f}$, which is equal to the tangent space of $\mathcal{C} c(K)$, has dimension at least 2 .

Let us consider the behavior of $\psi_{K}$ when $t_{2}$ and $t_{4}$ approach $t_{1}$ and $t_{3}$ respectively. Then Taylor's expansion formula implies

$$
\begin{aligned}
f\left(t_{1}\right) \wedge f\left(t_{2}\right) \wedge f\left(t_{3}\right) \wedge f\left(t_{4}\right)= & \left(t_{2}-t_{1}\right)\left(t_{4}-t_{3}\right) f\left(t_{1}\right) \wedge f\left(t_{1}^{\prime}\right) \wedge f\left(t_{3}\right) \wedge f^{\prime}\left(t_{3}\right) \\
& + \text { higher order terms. }
\end{aligned}
$$

Put $\nu_{f}^{(2,2)}\left(t_{1}, t_{3}\right)=f\left(t_{1}\right) \wedge f\left(t_{1}^{\prime}\right) \wedge f\left(t_{3}\right) \wedge f^{\prime}\left(t_{3}\right)$. Then $\nu_{f}^{(2,2)}\left(t_{1}, t_{3}\right)=0$ if and only if $f\left(t_{1}\right), f\left(t_{1}^{\prime}\right)$, $f\left(t_{3}\right)$, and $f^{\prime}\left(t_{3}\right)$ are linearly dependent as vectors in $\mathbb{R}^{5}$, which occurs if and only if $f^{\prime}\left(t_{3}\right)$ can be expressed as a linear combination of $f\left(t_{1}\right)-f\left(t_{3}\right)$ and $f\left(t_{1}^{\prime}\right)$. This is equivalent to the condition that $f^{\prime}\left(t_{3}\right)$, considered as a tangent vector at $f\left(t_{3}\right) \in S_{1}^{3}$, lies in the circle which can be obtained as the intersection of $S_{1}^{3}$ and the 3-dimensional vector subspace spanned by $f\left(t_{1}\right)-f\left(t_{3}\right)$ and $f\left(t_{1}^{\prime}\right)$. Therefore $\nu_{f}^{(2,2)}\left(t_{1}, t_{3}\right)=0$ if and only if $f\left(t_{1}\right), f^{\prime}\left(t_{1}\right), f\left(t_{3}\right)$, and $f^{\prime}\left(t_{3}\right)$ are concircular.

Put

$$
\mathcal{C} c^{(2,2)}(K)=\left\{(s, t) \in S^{1} \times S^{1} \backslash \triangle \mid f\left(t_{1}\right), f^{\prime}\left(t_{1}\right), f\left(t_{3}\right), \text { and } f^{\prime}\left(t_{3}\right) \text { are concircular }\right\} .
$$

Generically $\mathcal{C} c^{(2,2)}(K)$ is of dimension 0 since $(x, y)=(f(s), f(t))$ belongs to $\mathcal{C} c^{(2,2)}(K)$ if and only if $f^{\prime}(t)$ coincides with $v_{x}(y)$, the unit tangent vector at $x$ conformally translated to $y$. We 
remark that $\mathcal{C} c^{(2,2)}(K)$ has dimension 1 when $K$ is a $(p, q)$-torus knot which is an orbit of an $S^{1}$-action defined by

$$
S^{1} \ni e^{2 \pi i t} \mapsto\left(\mathbb{C}^{2} \supset S^{3} \ni(z, w) \mapsto\left(e^{2 \pi i p t} z, e^{2 \pi i q t} w\right) \in S^{3} \subset \mathbb{C}^{2}\right)
$$

Definition 4.2.5 The twice tangent sphere map $\psi_{K}^{(2,2)}$ for a knot $K$ which assigns the twice tangent sphere $\Sigma_{K}(f(s), f(t))$ to $(s, t) \in \operatorname{Conf}_{2}\left(S^{1}\right) \backslash \mathcal{C} c^{(2,2)}(K)$ is given by

$$
\begin{aligned}
\psi_{K}^{(2,2)}: \operatorname{Conf}_{2}\left(S^{1}\right) \backslash \mathcal{C} c^{(2,2)}(K) \ni(s, t) \mapsto \\
\quad \frac{\nu_{f}^{(2,2)}(s, t)}{\sqrt{L\left(\nu_{f}^{(2,2)}(s, t)\right)}}=\frac{f(s) \wedge f^{\prime}(s) \wedge f(t) \wedge f^{\prime}(t)}{\sqrt{L\left(f(s) \wedge f^{\prime}(s) \wedge f(t) \wedge f^{\prime}(t)\right)}} \in \Lambda \cong \mathcal{S} .
\end{aligned}
$$

Conjecture 4.2.6 If $K$ is non-trivial then the twice tangent sphere map $\psi_{K}^{(2,2)}$ is not injective, namely, there is a sphere $\Sigma$ that is tangent to $K$ at three points or more.

When $t$ approaches $s$, the twice tangent sphere approaches an osculating sphere that is the 2-sphere which is the most tangent to $K$ at $f(s)$. It contains the osculating circle and is tangent to $K$ in the fourth order. Taylor's expansion formula implies

$$
\nu_{f}^{(2,2)}(s, t)=\frac{1}{12}(t-s)^{4} f(s) \wedge f^{\prime}(s) \wedge f^{\prime \prime}(s) \wedge f^{\prime \prime \prime}(s)+\text { higher order terms. }
$$

Put $\nu_{f}^{(4)}(s)=f(s) \wedge f^{\prime}(s) \wedge f^{\prime \prime}(s) \wedge f^{\prime \prime \prime}(s)$. Suppose $\nu_{f}^{(4)}(s) \neq 0$. Lemma 2.2.3 (3) shows that $\nu_{f}^{(4)}(s)$ is not a time-like vector. If it is a light-like vector then 2.2.3 (2) implies that $\nu_{f}^{(4)}(s)=k f(s)$ for some $k \in \mathbb{R}$. Since $L(f(t), f(t))=0$ for any $t \in S^{1}$

$$
L\left(f(t), f^{\prime \prime}(t)\right)=-L\left(f^{\prime}(t), f^{\prime}(t)\right)=\sum_{i=1}^{4}\left(f_{i}^{\prime}(t)\right)^{2} \neq 0 \quad \text { for any } \quad t \in S^{1} .
$$

On the other hand as $L\left(\nu_{f}, f^{\prime \prime}(s)\right)=0$, we have $k=0$, which is a contradiction ${ }^{4}$. Therefore when $\nu_{f}^{(4)}(s) \neq 0$ the osculating sphere at $f(s)$ is given by $\nu_{f}^{(4)}(s) / \sqrt{\nu_{f}^{(4)}(s)}$.

\subsection{The real part as the canonical symplectic form on $T^{*} S^{3}$.}

In this subsection we show that the real part of the infinitesimal cross-ratio can be interpreted as the pull-back of the canonical symplectic form on the cotangent bundle $T^{*} S^{3}$ of the 3-sphere. This interpretation allows us to deduce the original definition (3) from the cosine formula in terms of the infinitesimal cross-ratio, (5).

\footnotetext{
${ }^{4}$ We assumed that $\left|f^{\prime}(t)\right|$ never vanishes.
} 


\subsubsection{The pull-back of the symplectic form to $S^{n} \times S^{n} \backslash \triangle$.}

Recall that a cotangent bundle $\pi: T^{*} M \rightarrow M$ of a manifold $M^{n}$ admits the canonical symplectic form $\omega_{0}$ given by

$$
\omega_{0}=\sum d p_{i} \wedge d q_{i}
$$

where $\left(p_{1}, \cdots, p_{n}\right)$ is a local coordinate of $M$ and $\left(q_{1}, \cdots, q_{n}\right)$ is the local coordinate of $T^{*} M$ defined by

$$
T_{x}^{*} M \ni v^{*}=\sum q_{i} d p_{i} .
$$

We remark that $\omega_{0}$ is an exact 2-form with $\omega_{0}=d\left(-\sum q_{i} d p_{i}\right)$.

Lemma 4.3.1 (1) There is a canonical bijection

$$
\psi_{n}: S^{n} \times S^{n} \backslash \triangle \rightarrow T^{*} S^{n} .
$$

(2) Let $\left(x_{1}, \cdots, x_{n+1}\right)$ be a system of local coordinates of

$$
U_{n+1}^{+}=\left\{\left(x_{1}, \cdots, x_{n+1}\right) \in S^{n} \subset \mathbb{R}^{n+1} \mid x_{n+1}>0\right\} .
$$

It determines the associated system of local coordinates of $\pi^{-1}\left(U_{n+1}^{+}\right) \subset T^{*} S^{n}$ by (6). The canonical bijection $\psi_{n}: S^{n} \times S^{n} \backslash \triangle \rightarrow T^{*} S^{n}$ is expressed with respect to this local coordinates as

$$
\psi_{n}\left(x\left(x_{1}, \cdots, x_{n+1}\right), y\left(y_{1}, \cdots, y_{n+1}\right)\right)=\left(x_{1}, \cdots, x_{n}, \frac{y_{1}-\frac{y_{n+1}}{x_{n+1}} x_{1}}{1-x \cdot y}, \cdots, \frac{y_{n}-\frac{y_{n+1}}{x_{n+1}} x_{n}}{1-x \cdot y}\right)
$$

on $U_{n+1}^{+} \times S^{n} \backslash \triangle$, where $x \cdot y$ denotes the inner product.

Proof: (1) Let $\Pi_{x}$ be the $n$-dimensional hyperplane in $\mathbb{R}^{n+1}$ passing through the origin that is perpendicular to $x \in S^{n}$, and $p_{x}: S^{n} \backslash\{x\} \rightarrow \Pi_{x}$ be the stereographic projection. We identify $\Pi_{x}$ with $T_{x} S^{n}$. Take an orthonormal bas $\left\{v_{1}, \cdots, v_{n}\right\}$ of $\Pi_{x} \cong T_{x} S^{n}$. Suppose $y \in S^{n} \backslash\{x\}$ is expressed as

$$
y=\tilde{y}_{1} v_{1}+\cdots+\tilde{y}_{n} v_{n}+\tilde{y}_{n+1} x
$$

with respect to the orthonormal basis $\left\{v_{1}, \cdots, v_{n}, x\right\}$ of $\mathbb{R}^{n+1}$. Then

$$
p_{x}(y)=\frac{\tilde{y}_{1}}{1-\tilde{y}_{n+1}} v_{1}+\cdots+\frac{\tilde{y}_{n}}{1-\tilde{y}_{n+1}} v_{n} .
$$

Put

$$
\psi_{x}(y)=\frac{\tilde{y}_{1}}{1-\tilde{y}_{n+1}} v_{1}^{*}+\cdots+\frac{\tilde{y}_{n}}{1-\tilde{y}_{n+1}} v_{n}^{*} \in T_{x}^{*} S^{n},
$$

where $\left\{v_{i}^{*}\right\}$ is the dual basis of $T_{x}^{*} S^{n}$. Then the map $\psi_{x}: S^{n} \backslash\{x\} \rightarrow T_{x}^{*} S^{n}$ does not depend on the choice of the orthonormal basis $\left\{v_{i}\right\}$ of $\Pi_{x} \cong T_{x} S^{n}$. Thus the map

$$
\psi_{n}: S^{n} \times S^{n} \backslash \triangle \ni(x, y) \mapsto\left(x, \psi_{x}(y)\right) \in T^{*} S^{n}
$$

makes the canonical bijection. 
Lemma 4.3.2 The pull-back $\omega=\psi_{n}{ }^{*} \omega_{0}$ of the canonical symplectic form $\omega_{0}$ of $T^{*} S^{n}$ by $\psi_{n}$ : $S^{n} \times S^{n} \backslash \triangle \rightarrow T^{*} S^{n}$ is given by

$$
\begin{aligned}
\omega & =d\left(-\frac{\sum_{i=1}^{n+1} y_{i} d x_{i}}{1-x \cdot y}\right)=d\left(\frac{\sum_{i=1}^{n+1} x_{i} d y_{i}}{1-x \cdot y}\right) \\
& =\frac{\sum_{i=1}^{n+1} d x_{i} \wedge d y_{i}}{1-x \cdot y}+\frac{\left(\sum_{i=1}^{n+1} y_{i} d x_{i}\right) \wedge\left(\sum_{i=1}^{n+1} x_{i} d y_{i}\right)}{(1-x \cdot y)^{2}}
\end{aligned}
$$

Proof: $\quad$ As $\omega_{0}=-d \sum q_{i} d p_{i}$

$$
\psi_{n}{ }^{*} \omega_{0}=-d \sum_{i=1}^{n} \frac{y_{i}-\frac{y_{n+1}}{x_{n+1}} x_{i}}{1-x \cdot y} d x_{i}
$$

on $U_{n+1}^{+} \times S^{n} \backslash \triangle$ by Lemma 4.3.1 (2). Since $-\sum_{i=1}^{n} x_{i} d x_{i}=x_{n+1} d x_{n+1}$ on $S^{n}$, it implies the formula.

Lemma 4.3.3 Let $p: S^{n} \backslash\{(0, \cdots, 0,1)\} \rightarrow \mathbb{R}^{n}$ be the stereographic projection. Put

$$
P_{n}=p^{-1} \times p^{-1}: \mathbb{R}^{n} \times \mathbb{R}^{n} \backslash \triangle \rightarrow S^{n} \times S^{n} \backslash \triangle \text {. }
$$

Then the pull-back $\omega_{\mathbb{R}^{n}}=P_{n}{ }^{*} \omega=P_{n}{ }^{*} \psi_{n}{ }^{*} \omega_{0}$ is given by

$$
\begin{aligned}
\omega_{\mathbb{R}^{n}} & =2 d\left(\frac{\sum\left(x_{i}-y_{i}\right) d y_{i}}{|x-y|^{2}}\right)=2 d\left(\frac{\sum\left(x_{i}-y_{i}\right) d x_{i}}{|x-y|^{2}}\right) \\
& =2\left(\frac{\sum d x_{i} \wedge d y_{i}}{|x-y|^{2}}-2 \frac{\left(\sum\left(x_{i}-y_{i}\right) d x_{i}\right) \wedge\left(\sum\left(x_{j}-y_{j}\right) d y_{j}\right)}{|x-y|^{4}}\right) .
\end{aligned}
$$

Proof: Suppose

$$
P_{n}\left(x\left(x_{1}, \cdots, x_{n}\right), y\left(y_{1}, \cdots, y_{n}\right)\right)=\left(X\left(X_{1}, \cdots, X_{n+1}\right), Y\left(Y_{1}, \cdots, Y_{n+1}\right)\right) .
$$

Then

$$
P_{n}^{*}\left(\frac{\sum_{i=1}^{n+1} X_{i} d Y_{i}}{1-X \cdot Y}\right)=2 \frac{\sum_{i=1}^{n}\left(x_{i}-y_{i}\right) d y_{i}}{|x-y|^{2}}+d \log \left(|y|^{2}+1\right)
$$

Therefore

$$
P_{n}^{*} \omega=P_{n}^{*} d\left(\frac{\sum_{i=1}^{n+1} X_{i} d Y_{i}}{1-X \cdot Y}\right)=2 d\left(\frac{\sum_{i=1}^{n}\left(x_{i}-y_{i}\right) d y_{i}}{|x-y|^{2}}\right) .
$$


Proposition 4.3.4 The 2-form $\omega_{\mathbb{R}^{n}}$ is invariant under the diagonal action of the conformal group $\mathcal{G}$ on $\mathbb{R}^{n} \times \mathbb{R}^{n} \backslash \triangle$ defined by

$$
g \cdot(x, y)=(g \cdot x, g \cdot y)
$$

where $g \in \mathcal{M}$ and $(x, y) \in \mathbb{R}^{n} \times \mathbb{R}^{n} \backslash \triangle$.

Proof: It is obvious that $\omega_{\mathbb{R}^{n}}$ is invariant under the diagonal action of multiplication by scalars $(x, y) \mapsto(c x, c y)$ and of addition of vectors, $(x, y) \mapsto(x+a, y+a)$. The previous Lemma implies that $\omega_{\mathbb{R}^{n}}$ is invariant under the diagonal action of the orthogonal group $O(n)$.

Therefore it suffices to show the invariance under the diagonal action $I \times I$ of the inversion $I$ with respect to the $(n-1)$-sphere with radius 1 whose center is the origin.

Suppose

$$
(I \times I)\left(x\left(x_{1}, \cdots, x_{n}\right), y\left(y_{1}, \cdots, y_{n}\right)\right)=\left(X\left(X_{1}, \cdots, X_{n}\right), Y\left(Y_{1}, \cdots, Y_{n}\right)\right) .
$$

Then

$$
(I \times I)^{*}\left(\frac{\sum\left(X_{i}-Y_{i}\right) d Y_{i}}{|X-Y|^{2}}\right)=\left(\frac{\sum\left(x_{i}-y_{i}\right) d y_{i}}{|x-y|^{2}}\right)+\frac{1}{2} d \log \left(|y|^{2}\right) .
$$

Therefore

$$
(I \times I)^{*} \omega_{\mathbb{R}^{n}}=(I \times I)^{*} 2 d\left(\frac{\sum\left(X_{i}-Y_{i}\right) d Y_{i}}{|X-Y|^{2}}\right)=2 d\left(\frac{\sum\left(x_{i}-y_{i}\right) d y_{i}}{|x-y|^{2}}\right)=\omega_{\mathbb{R}^{n}}
$$

We give another proof of the invariance.

Let $\Sigma_{1}$ be the $n$-sphere in $\mathbb{R}^{n+1}$ with center 0 and radius 1 , and $\Sigma_{\sqrt{2}}$ be the $n$-sphere in $\mathbb{R}^{n+1}$ with center $(0, \cdots, 0,1)$ and radius $\sqrt{2}$. Let $I_{\Sigma}$ be the inversions of $\mathbb{R}^{n+1} \cup\{\infty\}$ with respect to an $n$-sphere $\Sigma$ in $\mathbb{R}^{n+1}$.

Then

$$
p^{-1}(I(x))=I_{\Sigma_{\sqrt{2}}}\left(I_{\Sigma_{1}}(x)\right)=I_{I_{\Sigma_{\sqrt{2}}}\left(\Sigma_{1}\right)}\left(I_{\Sigma_{\sqrt{2}}}(x)\right)=I_{\mathbb{R}^{n}}\left(p^{-1}(x)\right)
$$

for $x \in \mathbb{R}^{n}$. Therefore

$$
P_{n} \circ(I \times I)=\left(I_{\mathbb{R}^{n}} \times I_{\mathbb{R}^{n}}\right) \circ P_{n}: \mathbb{R}^{n} \times \mathbb{R}^{n} \backslash \triangle \rightarrow S^{n} \times S^{n} \backslash \triangle .
$$

As

$$
\psi_{n} \circ\left(I_{\mathbb{R}^{n}} \times I_{\mathbb{R}^{n}}\right)=\psi_{n}: S^{n} \times S^{n} \backslash \triangle \rightarrow T^{*} S^{n},
$$

we get the conclusion:

$$
(I \times I)^{*} \omega_{\mathbb{R}^{n}}=(I \times I)^{*} P_{n}{ }^{*} \psi_{n}{ }^{*} \omega_{0}=\left(\psi_{n} \circ P_{n} \circ(I \times I)\right)^{*} \omega_{0}=\left(\psi_{n} \circ P_{n}\right)^{*} \omega_{0}=\omega_{\mathbb{R}^{n}}
$$


Lemma 4.3.5 Let

$$
\lambda=\frac{d w \wedge d z}{(w-z)^{2}}, \quad(w, z) \in \mathbb{C} \times \mathbb{C} \backslash \triangle
$$

be the 2-form on $\mathbb{C} \times \mathbb{C} \backslash \triangle \cong \mathbb{R}^{2} \times \mathbb{R}^{2} \backslash \triangle$ obtained as the infinitesimal cross-ratio of dw and $d z$. Then

(1) Both $\Re$ e $\lambda$ and $\Im m \lambda$ are exact 2-forms.

(2) $\Re e \lambda$ (or $\Im m \lambda$ ) is invariant (or invariant up to sign, respectively) under the diagonal action of the conformal group ${ }^{5}$ on $\mathbb{R}^{2} \times \mathbb{R}^{2} \backslash \triangle$.

Proof: As for the real part, since the next Lemma implies $\Re e \lambda=-\frac{1}{2} \omega_{\mathbb{R}^{2}}$, Lemma 4.3.3 and Proposition 4.3.4 show that $\Re e \lambda$ satisfies the desired properties.

As for the imaginary part, direct calculation shows

$$
\begin{aligned}
\Im m \lambda= & -2 \frac{\left(x_{1}-y_{1}\right)\left(x_{2}-y_{2}\right)\left(d x_{1} \wedge d y_{1}-d x_{2} \wedge d y_{2}\right)}{\left\{\left(x_{1}-y_{1}\right)^{2}+\left(x_{2}-y_{2}\right)^{2}\right\}^{2}} \\
& +\frac{\left\{\left(x_{1}-y_{1}\right)^{2}-\left(x_{2}-y_{2}\right)^{2}\right\}\left(d x_{1} \wedge d y_{2}+d x_{2} \wedge d y_{1}\right)}{\left\{\left(x_{1}-y_{1}\right)^{2}+\left(x_{2}-y_{2}\right)^{2}\right\}^{2}}
\end{aligned}
$$

(1) If we put

$$
\rho=\frac{\left(x_{1}-y_{1}\right) d x_{2}-\left(x_{2}-y_{2}\right) d x_{1}}{\left(x_{1}-y_{1}\right)^{2}+\left(x_{2}-y_{2}\right)^{2}}
$$

then $\Im m \lambda=-d \rho$.

(2) Let $I$ be the inversion with respect to the circle with radius 1 whose center is the origin. Then

$$
(I \times I)^{*} \rho=-\rho+d \arctan \left(\frac{x_{2}}{x_{1}}\right),
$$

which implies the invariance as in the proof of Proposition 4.3.4.

We remark that Lemma can be proved by a direct calculation with either complex or real coordinates.

Lemma 4.3.6 (Folklore) The real part of the infinitesimal cross-ratio 2-form is equal to minus one half of the pull-back of the canonical symplectic form of the cotangent bundle $T^{*} S^{2}$ :

$$
\Re e\left(\frac{d w \wedge d z}{(w-z)^{2}}\right)=-\frac{1}{2} \omega_{\mathbb{R}^{2}}=-\frac{1}{2} P_{2}^{*} \psi_{2}{ }^{*} \omega_{0} .
$$

\footnotetext{
${ }^{5}$ by conformal group we mean the group of transformations generated by the transformations $z \mapsto$ $\frac{a z+b}{c z+d}$ and $z \mapsto \bar{z}$ acting on the riemann sphere $\mathbb{C} \cup \infty$ (or the restriction of elements of this group to $\mathbb{R}^{2} \backslash\{$ the point where it is not defined, if necessary $\}$ )
} 
Proof: The left hand side is equal to

$$
\begin{aligned}
& \frac{\left\{\left(x_{1}-y_{1}\right)^{2}-\left(x_{2}-y_{2}\right)^{2}\right\}\left(d x_{1} \wedge d y_{1}-d x_{2} \wedge d y_{2}\right)}{\left\{\left(x_{1}-y_{1}\right)^{2}+\left(x_{2}-y_{2}\right)^{2}\right\}^{2}} \\
+ & 2 \frac{\left(x_{1}-y_{1}\right)\left(x_{2}-y_{2}\right)\left(d x_{1} \wedge d y_{2}+d x_{2} \wedge d y_{1}\right)}{\left\{\left(x_{1}-y_{1}\right)^{2}+\left(x_{2}-y_{2}\right)^{2}\right\}^{2}} \\
= & -\frac{d x_{1} \wedge d y_{1}+d x_{2} \wedge d y_{2}}{\left(x_{1}-y_{1}\right)^{2}+\left(x_{2}-y_{2}\right)^{2}} \\
+ & 2 \frac{\left\{\left(x_{1}-y_{1}\right) d x_{1}+\left(x_{2}-y_{2}\right) d x_{2}\right\} \wedge\left\{\left(x_{1}-y_{1}\right) d y_{1}+\left(x_{2}-y_{2}\right) d y_{2}\right\}}{\left\{\left(x_{1}-y_{1}\right)^{2}+\left(x_{2}-y_{2}\right)^{2}\right\}^{2}}
\end{aligned}
$$

which is equal to the right hand side.

\subsubsection{From cosine formula to the original definition of $E^{(2)}$.}

Now let us recall the definition of the infinitesimal cross-ratio. Let $\left(x_{0}, y_{0}\right)=\left(f\left(s_{0}\right), f\left(t_{0}\right)\right) \in$ $K \times K \backslash \triangle$. Then there is an oriented twice tangent sphere $\Sigma_{K}\left(x_{0}, y_{0}\right)$, which is given by $\psi_{K}^{(2,2)}\left(s_{0}, t_{0}\right)$ when $\left(s_{0}, t_{0}\right) \in \operatorname{Conf}_{2}\left(S^{1}\right) \backslash \mathcal{C} c^{(2,2)}(K)$, and otherwise by any oriented 2-sphere that contains the circle $C\left(x_{0}, x_{0}, y_{0}\right)$. Let $T_{0}$ be a conformal transformation of $\mathbb{R}^{3} \cup\{\infty\}$ that maps $\Sigma_{K}\left(x_{0}, y_{0}\right)$ to $\mathbb{R}^{2} \times\{0\} \subset \mathbb{R}^{3}$ preserving the orientations. Let

$$
\frac{d w \wedge d z}{(w-z)^{2}}
$$

be a complex valued 2-form on

$$
\mathbb{R}^{3} \times \mathbb{R}^{3} \backslash \triangle \cong\left\{\left(\left(w, x_{3}\right),\left(z, y_{3}\right)\right) \in(\mathbb{C} \times \mathbb{R}) \times(\mathbb{C} \times \mathbb{R}) \backslash \triangle\right\} .
$$

Then, as a 2-form, the infinitesimal cross-ratio $\Omega_{C R}$ satisfies

$$
\Omega_{C R}\left(x_{0}, y_{0}\right)=\left(T_{0} \times T_{0}\right)^{*}\left(\frac{d w \wedge d z}{(w-z)^{2}}\right)\left(x_{0}, y_{0}\right) .
$$

Lemma 4.3.7 Let $K=f\left(S^{1}\right)$ be a knot. Then the real part of the infinitesimal cross-ratio $\Omega_{C R}$ defined in definition 4.1 .3 is equal to minus one half of the pull-back of the canonical symplectic form of the cotangent bundle $T^{*} S^{3}$ :

$$
\cos \theta \frac{d x d y}{|x-y|^{2}}=\Re e \Omega_{C R}=-\left.\frac{1}{2} \omega_{\mathbb{R}^{3}}\right|_{K \times K \backslash \triangle}=-\frac{1}{2}\left(\iota_{K} \times \iota_{K}\right)^{*} P_{3}{ }^{*} \psi_{3}{ }^{*} \omega_{0},
$$

where $\iota_{K}: K \rightarrow \mathbb{R}^{3}$ is the inclusion map.

Proof: We show that their pull-backs $(f \times f)^{*} \Re e \Omega_{C R}$ and $-\frac{1}{2}(f \times f)^{*} \omega_{\mathbb{R}^{3}}$ coincide at any given $\left(s_{0}, t_{0}\right) \in S^{1} \times S^{1} \backslash \triangle$. 
Proposition 4.3 .4 implies

$$
-\frac{1}{2}(f \times f)^{*} \omega_{\mathbb{R}^{3}}=-\frac{1}{2}\left(\left(T_{0} \circ f\right) \times\left(T_{0} \circ f\right)\right)^{*} \omega_{\mathbb{R}^{3}} .
$$

On the other hand, since

$$
\begin{aligned}
& \left(\left(T_{0} \circ f\right)^{*} d x_{3}\right)\left(s_{0}, t_{0}\right)=\left(\frac{d}{d s}\left(T_{0} \circ f\right)_{3}\right)\left(s_{0}\right), \\
& \left(\left(T_{0} \circ f\right)^{*} d y_{3}\right)\left(s_{0}, t_{0}\right)=\left(\frac{d}{d t}\left(T_{0} \circ f\right)_{3}\right)\left(t_{0}\right)
\end{aligned}
$$

we have

$$
\begin{aligned}
& -\frac{1}{2}\left(\left(T_{0} \circ f\right) \times\left(T_{0} \circ f\right)\right)^{*} \omega \mathbb{R}^{3}\left(s_{0}, t_{0}\right) \\
& =\left(\left(T_{0} \circ f\right) \times\left(T_{0} \circ f\right)\right)^{*}\left(-\frac{\sum_{i=1}^{3} d x_{i} \wedge d y_{i}}{|x-y|^{2}}\right. \\
& \left.+2 \frac{\left(\sum_{i=1}^{3}\left(x_{i}-y_{i}\right) \wedge d x_{i}\right) \wedge\left(\sum_{i=1}^{3}\left(x_{i}-y_{i}\right) \wedge d y_{i}\right)}{|x-y|^{4}}\right)\left(s_{0}, t_{0}\right) \\
& =\left(\left(T_{0} \circ f\right) \times\left(T_{0} \circ f\right)\right)^{*}\left(-\frac{\sum_{i=1}^{2} d x_{i} \wedge d y_{i}}{|x-y|^{2}}\right. \\
& \left.+2 \frac{\left(\sum_{i=1}^{2}\left(x_{i}-y_{i}\right) \wedge d x_{i}\right) \wedge\left(\sum_{i=1}^{2}\left(x_{i}-y_{i}\right) \wedge d y_{i}\right)}{|x-y|^{4}}\right)\left(s_{0}, t_{0}\right) \\
& =(f \times f)^{*}\left(T_{0} \times T_{0}\right)^{*} \Re\left(\frac{d w \wedge d z}{(w-z)^{2}}\right)\left(s_{0}, t_{0}\right)=(f \times f)^{*} \Re e \Omega_{C R}\left(s_{0}, t_{0}\right) .
\end{aligned}
$$

Put for $0<\epsilon \ll 1$

$$
N(\epsilon)=\left\{(s, t) \in S^{1} \times S^{1}|\epsilon \leq| s-t \mid \leq 1-\epsilon\right\},
$$

where $S^{1}=[0,1] / \sim$, and assume $N(\epsilon)$ has a natural orientation as a subspace of the torus. Then its boundary $\partial N(\epsilon)$ consists of two closed curves $L_{+}$and $L_{-}$with opposite orientations, where

$$
L_{ \pm}=\left\{(s, t) \in S^{1} \times S^{1} \mid t-s= \pm \epsilon(\bmod \mathbb{Z})\right\} .
$$

Then

$$
\begin{aligned}
E^{(2)}(K) & =\iint_{S^{1} \times S^{1} \backslash \triangle}(f \times f)^{*}\left(\frac{d x d y}{|x-y|^{2}}-\Re e \Omega_{C R}\right)+4 \\
& =\lim _{\epsilon \rightarrow 0} \iint_{N(\epsilon)}\left(\frac{d s d t}{|f(s)-f(t)|^{2}}-(f \times f)^{*} \Re \Omega_{C R}\right)+4 .
\end{aligned}
$$


On the other hand, as

$$
-(f \times f)^{*} \Re e \Omega_{C R}=\frac{1}{2}(f \times f)^{*} \omega_{\mathbb{R}^{3}}=d(f \times f)^{*}\left(\frac{\sum\left(x_{i}-y_{i}\right) d y_{i}}{|x-y|^{2}}\right)
$$

by Lemmas 4.3.7 and 4.3.3, there holds

$$
E^{(2)}(K)=\lim _{\epsilon \rightarrow 0}\left\{E_{\epsilon}^{(2)}(K)+\int_{L_{+} \cup L_{-}}(f \times f)^{*}\left(\frac{\sum\left(x_{i}-y_{i}\right) d y_{i}}{|x-y|^{2}}\right)\right\}+4
$$

by Stokes' theorem. As

$$
\left(f(s)-f(t), f^{\prime}(t)\right)=(s-t)+O\left(|s-t|^{3}\right),
$$

we have

$$
\begin{gathered}
\int_{L_{+} \cup L_{-}}(f \times f)^{*}\left(\frac{\sum\left(x_{i}-y_{i}\right) d y_{i}}{|x-y|^{2}}\right)=\int_{L_{+} \cup L_{-}} \frac{\left(f(s)-f(t), f^{\prime}(t)\right)}{|f(s)-f(t)|^{2}} d t \\
=\int_{0}^{1} \frac{-\epsilon+O\left(\epsilon^{3}\right)}{\epsilon^{2}+O\left(\epsilon^{4}\right)} d t+\int_{1}^{0} \frac{\epsilon+O\left(\epsilon^{3}\right)}{\epsilon^{2}+O\left(\epsilon^{4}\right)} d t=-\frac{2}{\epsilon}+O(\epsilon) .
\end{gathered}
$$

Therefore we restored the original definition (3):

$$
E^{(2)}(K)=\lim _{\epsilon \rightarrow 0}\left(E_{\epsilon}^{(2)}(K)-\frac{2}{\epsilon}\right)+4
$$

\section{The (absolute) imaginary cross-ratio energy, $E_{|\sin \theta|} \cdot$}

In the previous section we introduced the infinitesimal cross-ratio $\Omega_{C R}$, which is the unique conformal invariant of a pair of infinitesimal curve segments $d x$ and $d y$, and showed that $E^{(2)}$ can be expressed in terms of the absolute value and the real part of if. On the other hand, one can use the imaginary part of $\Omega_{C R}$.

\subsection{The projection of the inverted open knot.}

Definition 5.1.1 The absolute imaginary cross-ratio energy or the conformal $|\sin \theta|$ energy, $E_{|\sin \theta|}$, is defined by

$$
E_{|\sin \theta|}(K)=\iint_{K \times K \backslash \triangle}\left|\Im m \Omega_{C R}\right|=\iint_{K \times K \backslash \triangle} \frac{|\sin \theta| d x d y}{|x-y|^{2}}
$$

$E_{|\sin \theta|}$ is well-defined since the argument of cross-ratio, $\theta$, is of the same order as $|x-y|^{2}$ near the diagonal set by the following Lemma.

The interest of the $E_{|\sin \theta|}$ energy has already been noticed by Kusner and Sullivan [Ku-Su], section 4.

Remark: The lemma 2.2.7 shows that the $\operatorname{sign}$ of $\sin \theta$ is constant, the absolute value allows us to forget it. 
Lemma 5.1.2 If a knot is of class $C^{4}$, the conformal angle $\theta_{K}(x, y)$ satisfies

$$
\theta_{K}(x, y)=\frac{\sqrt{k^{2} \tau^{2}+k^{\prime 2}}}{6}|x-y|^{2}+O\left(|x-y|^{3}\right),
$$

near the diagonal set, where $k=\left|f^{\prime \prime}\right|$ is the curvature and $\tau$ is the torsion of the knot at $x$.

Proof: Let us first remark that the unit tangent vector at $x$ conformally translated to $y$, $v_{x}(y)$, is expressed as

$$
v_{x}(y)=2\left(f^{\prime}(s), \frac{f(t)-f(s)}{|f(t)-f(s)|}\right) \cdot \frac{f(t)-f(s)}{|f(t)-f(s)|}-f^{\prime}(s) .
$$

since $v_{x}(y)$ is symmetric to $f^{\prime}(s)$ with respect to $f(t)-f(s)$.

Assume that a knot $f$ is of class $C^{4}$ and parametrized by the arc-length. Put $s=0$ and take the Frenet frame at $x$. Then by Bouquet's formula $f(t)$ is expressed as

$$
f_{1}(t)=t-\frac{k^{2}}{6} t^{3}+\cdots, \quad f_{2}(t)=\frac{k}{2} t^{2}+\frac{k^{\prime}}{6} t^{3}+\cdots, \quad f_{3}(t)=\frac{k \tau}{6} t^{3}+\cdots .
$$

Then as $f^{\prime}(0)=(0,0,1)$ the unit tangent vector at $x$ conformally translated to $y$ is given by

$$
\begin{aligned}
v_{x}(y) & =\frac{\left(f_{1}^{2}(t)-f_{2}^{2}(t)-f_{3}^{2}(t), 2 f_{1}(t) f_{2}(t), 2 f_{1}(t) f_{3}(t)\right)}{|f(t)|^{2}} \\
& =\frac{\left(t^{2}-\frac{7 k^{2}}{12} t^{4}+O\left(t^{5}\right), k t^{3}+\frac{k^{\prime}}{3} t^{4}+O\left(t^{5}\right), \frac{k \tau}{3} t^{4}+O\left(t^{5}\right)\right)}{t^{2}\left(1-\frac{k^{2}}{12} t^{2}+O\left(t^{3}\right)\right)} .
\end{aligned}
$$

On the other hand

$$
\frac{f^{\prime}(t)}{\left|f^{\prime}(t)\right|}=\frac{\left(1-\frac{k^{2}}{2} t^{2}+O\left(t^{3}\right), k t+\frac{k^{\prime}}{2} t^{2}+O\left(t^{3}\right), \frac{k \tau}{2} t^{2}+O\left(t^{3}\right)\right)}{1+O\left(t^{3}\right)} .
$$

Hence

$$
\begin{aligned}
|\sin \theta| & =\left|\frac{f^{\prime}(t)}{\left|f^{\prime}(t)\right|} \times v_{x}(y)\right|=\frac{\left|\left(O\left(t^{5}\right), \frac{k \tau}{6} t^{4}+O\left(t^{5}\right),-\frac{k^{\prime}}{6} t^{4}+O\left(t^{5}\right)\right)\right|}{t^{2}\left(1-\frac{k^{2}}{12} t^{2}+O\left(t^{3}\right)\right)\left(1+O\left(t^{3}\right)\right)} \\
& =\frac{\sqrt{k^{2} \tau^{2}+k^{\prime 2}}}{6} t^{2}+O\left(t^{3}\right) .
\end{aligned}
$$

Let us give a geometric interpretation of $E_{|\sin \theta|}$ using the inverted open knots. Put

$$
V_{|\sin \theta|}(K ; x)=\int_{K} \frac{|\sin \theta| d y}{|x-y|^{2}}
$$


Let $I_{x}, \tilde{K}_{x}=I_{x}(K)$, and $\tilde{y}=I_{x}(y)$ be as in subsection 3.3. Let $\Pi_{x}$ be the normal plane to $K$ at $x, n=-f^{\prime}(s)$ be the normal vector to $\Pi_{x}$, and $\pi_{x}: \mathbb{R}^{3} \rightarrow \Pi_{x}$ be the orthogonal projection. Since $I_{x *}\left(v_{x}(y)\right)=n$ the absolute value of the argument of cross-ratio $\theta_{K}(x, y)$ is equal to the angle between $n$ and the tangent vector to $\tilde{K}_{x}$ at $\tilde{y}$. Hence

$$
V_{|\sin \theta|}(K ; x)=\int_{K} \frac{|\sin \theta| d y}{|x-y|^{2}}=\int_{\tilde{K}_{x}}|\sin \theta||d \tilde{y}|
$$

is the length of the projection $\pi_{x}\left(\tilde{K}_{x}\right) \subset \Pi_{x}$. 

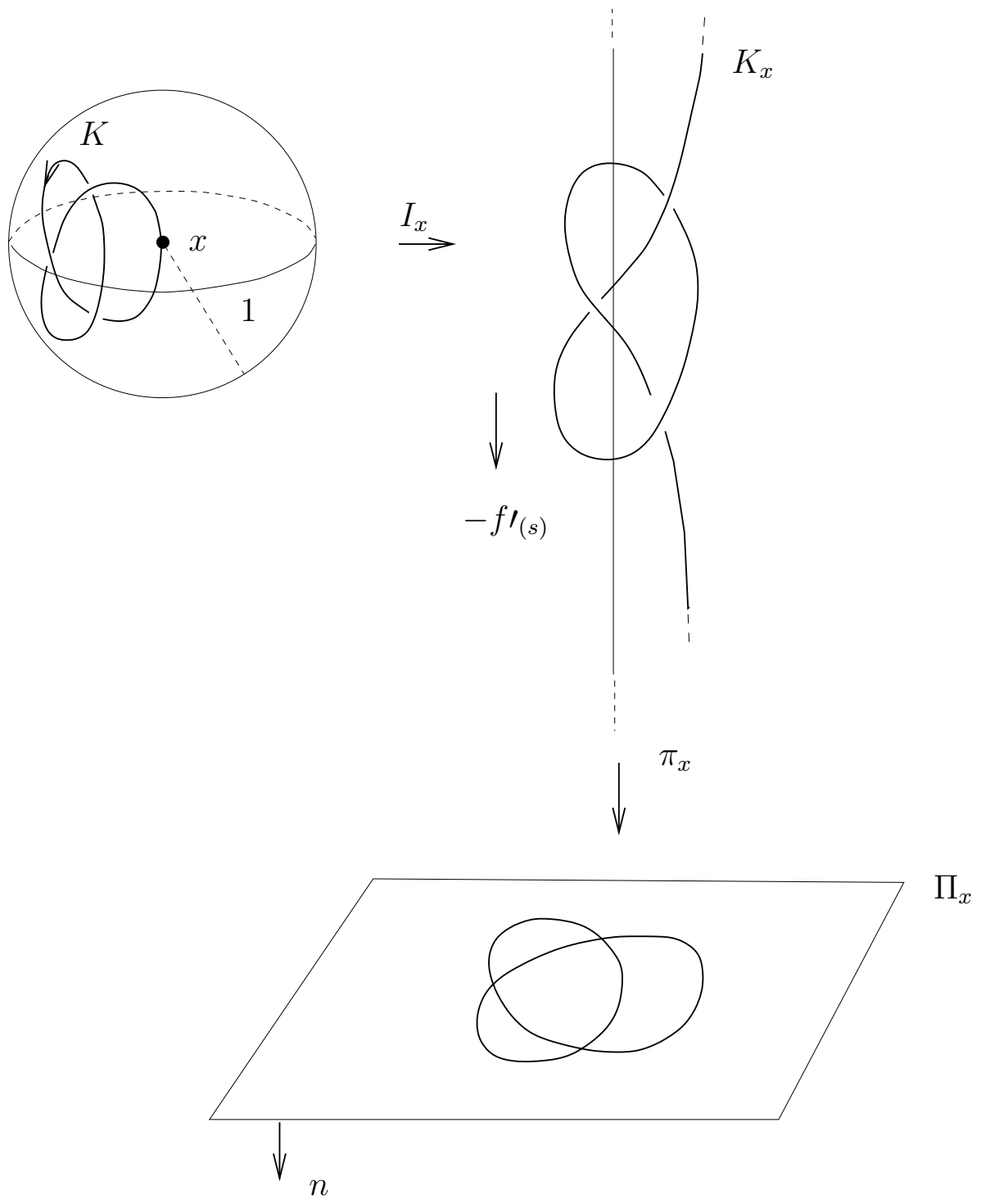

Figure 6: The projection of the inverted open knot.

Thus the imaginary cross-ratio energy is equal to the total length of the projection of the inverted open knots:

$$
E_{|\sin \theta|}(K)=\int_{K} \operatorname{Length}\left(\pi_{x}\left(I_{x}(K)\right)\right) d x
$$

\subsection{The properties of $E_{|\sin \theta|}$.}

We show first that $E_{|\sin \theta|}$ can detect the unknot.

Example 5.2.1 Let $K_{\circ}$ be the standard planar circle. Then $\theta_{K_{\circ}}(x, y)=0$ for any $(x, y)$. Therefore $E_{|\sin \theta|}\left(K_{\circ}\right)=0$.

We remark that Claim 4.1 .4 implies that $E_{|\sin \theta|}(K)=0$ if and only if $K=K_{\circ}$. 
Theorem 5.2.2 ([Lin]

The imaginary cross-ratio energy crossing number from above, namely,

$$
E_{|\sin \theta|}(K) \geq 4 \pi a c(K)
$$

for any knot $K$.

Corollary 5.2.3 If $K$ is a non-trivial knot then $E_{|\sin \theta|}(K) \geq 12 \pi$.

Proof: Assume that a knot is parametrized by the arc-length. Put

$$
u=f^{\prime}(s), v=f^{\prime}(t), w=\frac{f(t)-f(s)}{|f(t)-f(s)|}, \quad \text { and } \quad \tilde{u}=v_{f(s)}(f(t)) .
$$

Recall that $\tilde{u}=2(u, w) w-u$, and that the conformal angle $\theta$ is the angle between $\tilde{u}$ and $v$.

Suppose $\theta \neq 0, \pi$. Then the tangent plane $T_{f(t)} \Sigma$ at $f(t)$ to the twice tangent spere $\Sigma=$ $\Sigma_{K}(f(s), f(t))$ is spanned by $\tilde{u}$ and $v$. Let $\psi=\psi(s, t)$ be the angle between the chord joining $f(t)$ and $f(s)$ and the tangent plane $T_{f(t)} \Sigma$.

Then the numerator of the integrand of the average crossing number (which is the absolute value of the integrand in Gauss formula) is:

$$
|(u \times v, w)|=|(\tilde{u} \times v, w)|=|\sin \psi||\sin \theta|
$$

Therefore

$$
a c(K)=\frac{1}{4 \pi} \iint_{S^{1} \times S^{1}} \frac{|\sin \psi(s, t)||\sin \theta(f(s), f(t))|}{|f(s)-f(t)|^{2}} d s d t \leq \frac{1}{4 \pi} E_{|\sin \theta|}(K) .
$$

When $\theta=0$ or $\pi$ then, $|(u \times v, w)|=\sin \theta=0$.

Next we show that $E_{|\sin \theta|}$ is an energy functional for knots in the weak sense.

Theorem 5.2.4 For any real numbers $b, \delta\left(0<\delta \leq \frac{1}{2}\right)$, and $\kappa_{0}$, there exists a positive constant $C=C\left(b, \delta, \kappa_{0}\right)$ such that if a knot $K$ with length $l(K)$ whose curvature is not greater than $\kappa_{0}$ has a pair of points $x, y$ on it which satisfy that $\delta_{K}(x, y)=\delta l(K)$ and that $|x-y| \leq C l(K)$, then $E_{|\sin \theta|}(K) \geq b$.

Proof: $\operatorname{Fix} \delta$ and $\kappa_{0}\left(\kappa_{0} \geq 2 \pi\right)$, and let $0<d \leq \delta$. Put

$$
\mathcal{K}(d)=\mathcal{K}_{\delta, \kappa_{0}}(d)=\left\{\begin{array}{l|l}
K: \text { a knot } & \begin{array}{l}
\text { The length of } K \text { is } 1 . \\
\text { The curvature of } K \text { is not greater than } \kappa_{0} . \\
\exists x, y \in K \text { such that } \\
\text { i) the shorter arc-length between } x \text { and } y \text { is } \delta, \\
\text { ii) }|x-y| \leq d .
\end{array}
\end{array}\right\}
$$

We show

$$
\lim _{d \rightarrow+0}\left(\inf _{K \in \mathcal{K}(d)} E_{|\sin \theta|}(K)\right)=\infty
$$


Lemma 5.2.5 Let

$$
d_{0}=\min \left\{\frac{1}{100}, \frac{\delta^{2}}{5},\left(\frac{-1+\sqrt{1+\frac{1}{50 \kappa_{0}}}}{2}\right)^{2}\right\} .
$$

Let $d \leq d_{0}$. Suppose $K=f\left(S^{1}\right) \in \mathcal{K}(d)$ is parametrized by arc-length and satisfies that $|f(0)-f(\delta)|=d$. If $5 d \leq t \leq \sqrt{d}$ then at least one of

$$
V_{|\sin \theta|}(K ; f( \pm t)), V_{|\sin \theta|}(K ; f(\delta \pm t))
$$

is greater than

$$
\frac{1}{100} \cdot \frac{1}{d+t}
$$

We show that Lemma implies Theorem. Since $10 d \leq \sqrt{d}<\frac{\delta}{2}$

$$
f([-\sqrt{d},-5 d]) \sqcup f([5 d, \sqrt{d}]) \sqcup f([\delta+5 d, \delta+\sqrt{d}]) \sqcup f([\delta-\sqrt{d}, \delta-5 d])
$$

is a disjoint union of curve segments of $K$ where $S^{1}$ is regarded as $\mathbb{R}$ modulo $\mathbb{Z}$. Since $V_{|\sin \theta|}(K ; y) \geq 0$ for any $y \in K$,

$$
\begin{aligned}
E_{|\sin \theta|}(K) \geq & \int_{5 d}^{\sqrt{d}}\left\{V_{|\sin \theta|}(K ; f(-t))+V_{|\sin \theta|}(K ; f(t))\right. \\
& \left.+V_{|\sin \theta|}(K ; f(\delta-t))+V_{|\sin \theta|}(K ; f(\delta+t))\right\} d t \\
\geq & \int_{5 d}^{\sqrt{d}} \frac{1}{100} \cdot \frac{1}{d+t} d t=\frac{1}{100}\left\{\log \left(1+\frac{1}{\sqrt{d}}\right)-\log 6\right\},
\end{aligned}
$$

which explodes as $d$ goes down to 0 , which completes the proof of theorem 5.2.4.

Proof of Lemma 5.2.5: Assume there is a $t$ with $5 d \leq t \leq \sqrt{d}$ such that

$$
V_{|\sin \theta|}(K ; f( \pm t)), V_{|\sin \theta|}(K ; f(\delta \pm t)) \geq \frac{1}{100} \cdot \frac{1}{d+t} .
$$

Put $x_{ \pm}=f( \pm t)$. Let $R_{t}=1 /\left|f^{\prime \prime}(t)\right|$ be the radius of curvature at $x_{+}$, and $C\left(x_{+}, x_{+}, x_{+}\right)$, $\tilde{K}_{x_{+}}=I_{x_{+}}(K), \Pi_{x_{+}}$, and $\pi_{x_{+}}$be as before. Define $x_{+}^{\prime}$ by

$$
C\left(x_{+}, x_{+}, x_{+}\right) \cap \Pi_{x_{+}}=\left\{x_{+}, x_{+}^{\prime}\right\} .
$$

Let $\hat{x}_{+}=I_{x_{+}}\left(x_{+}^{\prime}\right)$. Then

$$
\lim _{K \backslash\left\{x_{+}\right\} \ni y \rightarrow x_{+}} \pi_{x_{+}}(y)=\hat{x}_{+}
$$

Since

$$
V_{|\sin \theta|}\left(K ; x_{+}\right)=\operatorname{Length}\left(\pi_{x_{+}}\left(\tilde{K}_{x_{+}}\right)\right) \leq \frac{1}{100} \cdot \frac{1}{d+t}
$$



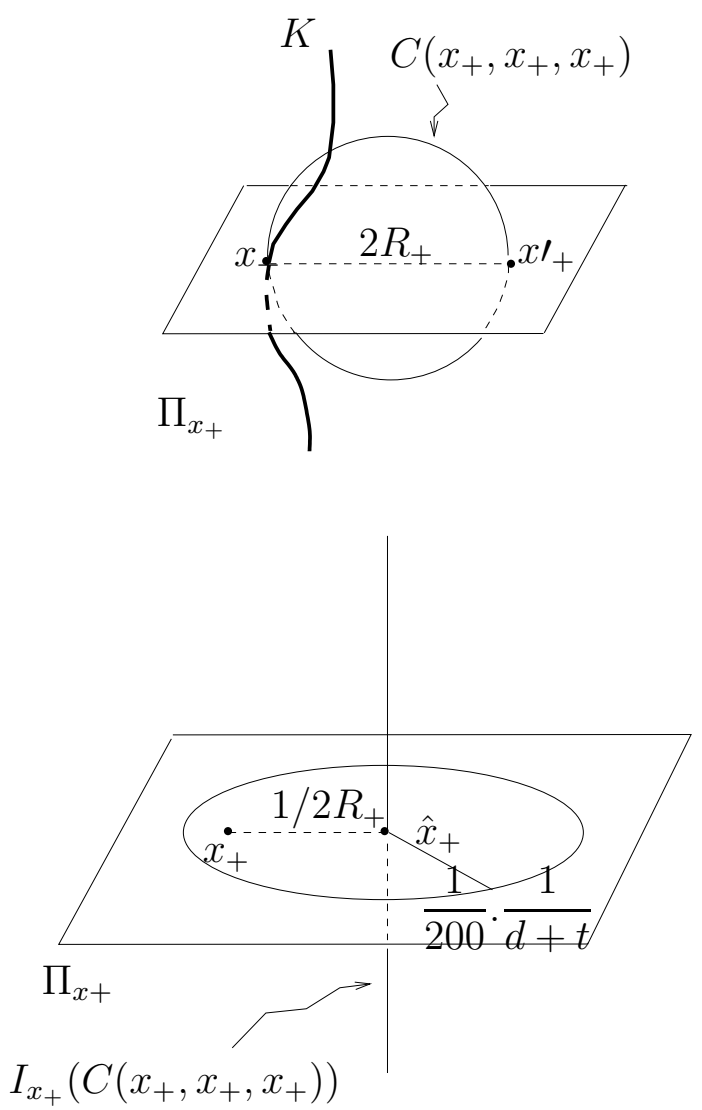

Figure 7: The osculating circle, $x_{+}, x_{+}^{\prime}$, and $\hat{x}_{+}$.

$\pi_{x_{+}}\left(\tilde{K}_{x_{+}}\right)$lies inside the circle on $\Pi_{x_{+}}$with center $x_{+}$and radius $1 /(200(d+t))$. Since

$$
\sqrt{d} \leq \frac{-1+\sqrt{1+\frac{1}{50 \kappa_{0}}}}{2}
$$

there holds

$$
\left|x_{+}-\hat{x}_{+}\right|=\frac{1}{2 R_{t}} \leq \frac{1}{2} \kappa_{0} \leq \frac{1}{2} \cdot \frac{1}{200} \cdot \frac{1}{d+\sqrt{d}} \leq \frac{1}{400} \cdot \frac{1}{d+t} .
$$

Therefore $\pi_{x_{+}}\left(\tilde{K}_{x_{+}}\right)$lies inside the circle $\Gamma$ on $\Pi_{x_{+}}$with center $x_{+}$and radius $3 /(400(d+t))$. This means that $\tilde{K}_{x_{+}}$lies inside the cylinder $D^{2} \times \mathbb{R}$, where $\partial D^{2}=\Gamma$ and the direction of $\mathbb{R}$ is $f^{\prime}(t)$. If we apply the inversion $I_{x_{+}}$again, this implies that $K$ lies outside the "degenerate ${ }^{6}$ open solid torus" $N_{t}$ whose meridian disc has radius $(400(d+t)) / 3$, as illustrated in Figure 8 , The same argument applies to $-t$ to conclude that $K$ lies outside the "degenerate open solid torus" $N_{-t}$. Since

$$
|f(t)-f(-t)| \leq 2 t \ll \frac{400}{3}(d+t)
$$

\footnotetext{
${ }^{6}$ By a "degenerate solid torus" we mean a torus of revolution of a circle around a tangent line.
} 

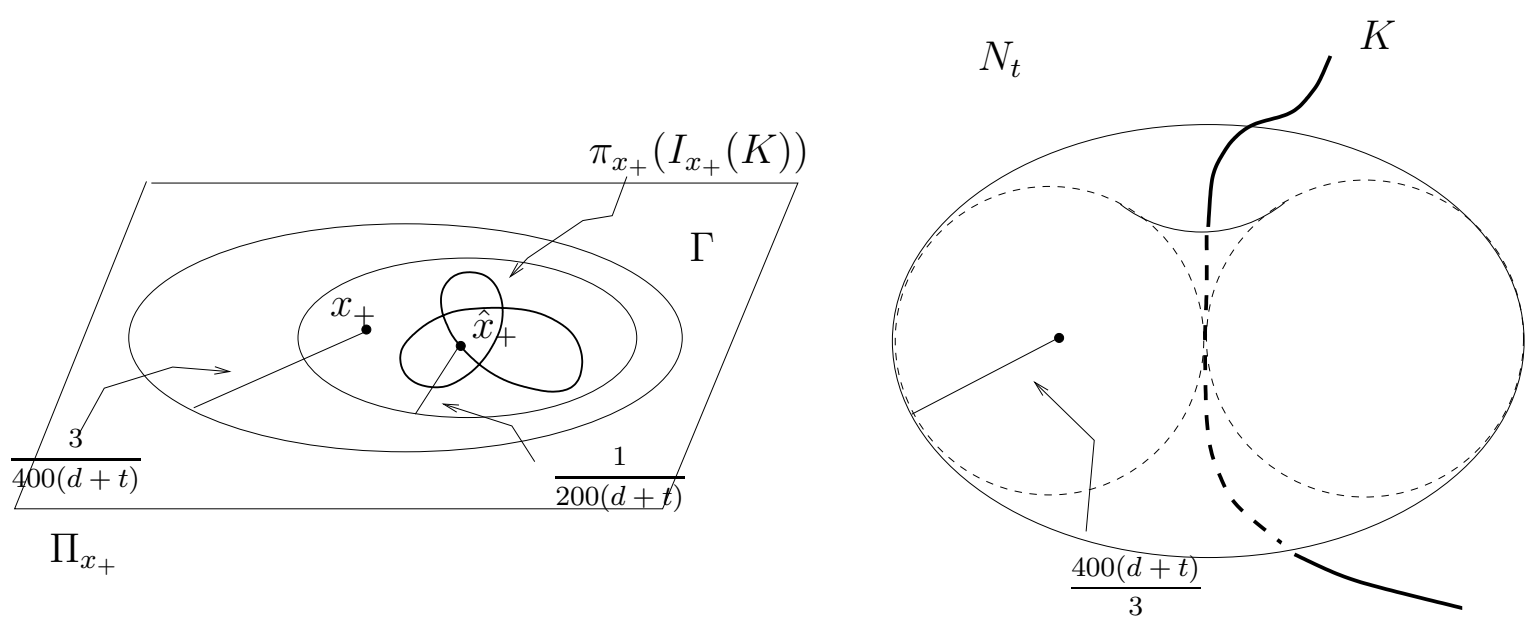

Figure 8: The circle $\Gamma$ and the degenerate solid torus.

$f([-t, t])$ is contained in a region $D$ bounded by $N_{t}$ and $N_{-t}$ as illustrated in Figure 9$]^{7}$

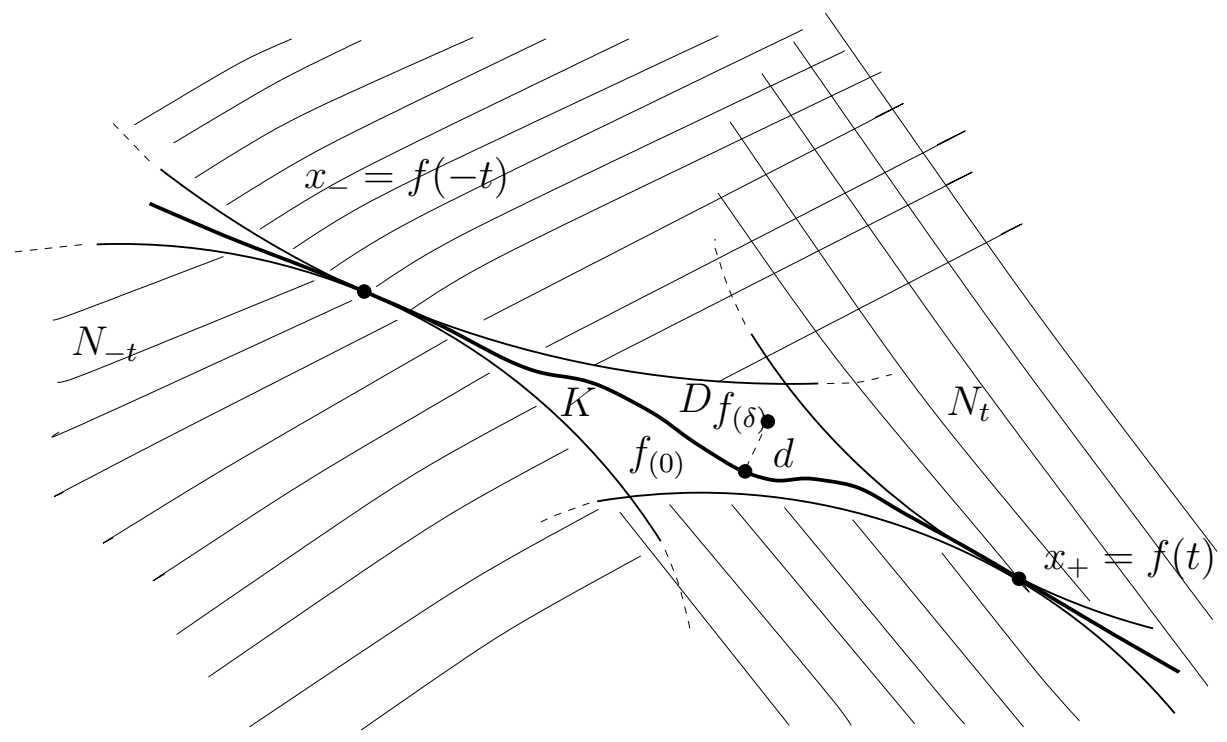

Figure 9: The domain $D$ illustrated as a 2-dimensional figure.

Sublemma 5.2.6 $f(\delta)$ is contained in $D$.

We show that Sublemma implies Lemma 5.2.5. If $f(\delta)$ is contained in $D$, then the knot $K$ must have double points at $x_{+}$and $x_{-}$, which is a contradiction. (End of the proof of Lemma 5.2 .5 )

${ }^{7} D$ is the bounded component of $\mathbb{R}^{3} \backslash\left(N_{t} \cup N_{-t}\right)$. 
Proof of Sublemma 5.2.6; Since $|f(0)-f(\delta)|=d$ which is much smaller than the radius $(400(d+t)) / 3$ of the meridian discs of the degenerate solid tori $N_{t}$ and $N_{-t}$, it suffices to show that

$$
|f(0)-f( \pm t)|>d
$$

to prove Sublemma. First we remark that

$$
t \leq \sqrt{d} \leq \frac{-1+\sqrt{1+\frac{1}{50 \kappa_{0}}}}{2}<\frac{1}{200 \kappa_{0}}
$$

For $0<s \leq t$

$$
\frac{d}{d s}\left(f^{\prime}(0), f^{\prime}(s)\right)=\left(f^{\prime}(0), f^{\prime \prime}(s)\right) \leq \kappa_{0}
$$

hence

$$
\left(f^{\prime}(0), f^{\prime}(s)\right) \leq 1-\kappa_{0} s
$$

As $s \leq \frac{1}{\kappa_{0}}$ we have

$$
\mid f(0)-g(t) \geq \int_{0}^{t}\left(1-\kappa_{0} s\right) d s=t\left(1-\frac{\kappa_{0}}{2} t\right)>d .
$$

\section{Measure of non-trivial spheres.}

\subsection{Spheres of dimension 0}

We will start with spheres of dimension 0 in $S^{1}$, and study their positions with respect to a "torus" $T$ made of 4 distinct points.

Notice that, if the conformal image of four points is $(-R,-1,1, R)$, the cross ratio is $R$. We will use this remark later, to give an interpretation of the modulus of a ring or of the zone between two non-intersecting spheres.

An oriented sphere $\sigma$ disjoint from $T$ bounds an interval I. We will say that $\sigma$ is non-trivial if I contains two points of $T$. Informally we may say that the small enough spheres will all be trivial.

Proposition 6.1.1 The "torus" $T$ which minimizes the measure of the set of the non-trivial spheres is the "torus" made of the four vertices of a square (or its image by the conformal group of the circle).

The domain $Z$ of $\mathcal{S}$ formed by the non-trivial spheres is bounded by segments of light rays formed by the spheres containing one of the four points of $T$.

As the only conformal invariant of a set of four points is their cross-ratio, The measure $m(Z)$ is a function of this cross-ratio.

Proof: The proof of the proposition is a computation. Using the stereographic projection of $S^{1}$ on $\mathbb{R}$ the measure on $\mathcal{S}=\left\{\right.$ pairs of points of $\left.S^{1}\right\}$ is $\frac{2}{(y-x)^{2}}|d x \wedge d y|$. Without loss of 


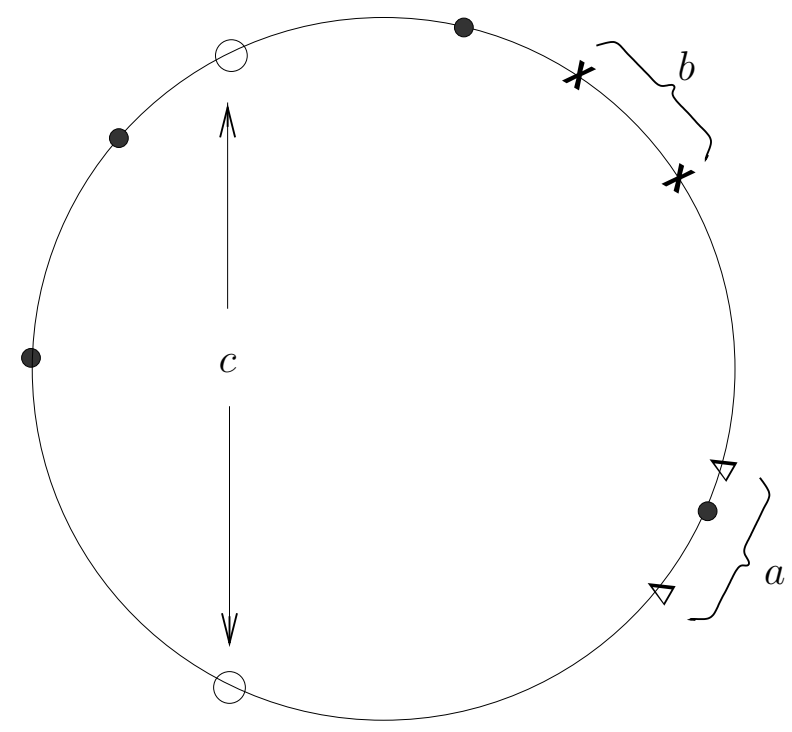

Figure 10: A non-trivial (c), and two trivial (a and b) 0-spheres.

generality, we can suppose that the four points of the "torus" $T$ are $\{\infty, 0,1, z\}$. We will make the computation of the measure of "half" of the points of $Z$, that is $\{\infty<x<0 ; 1<y<z\}$, supposing $z>1$. The other cases are analogous. One has $m\left(Z_{z}\right)=m(\{\infty<x<0 ; 1<y<$ $z\})+m(\{0<x<1 ; z<y<\infty\})$. One has :

$$
m(\{\infty<x<0 ; 1<y<z\})=\int_{1<y<z} \int_{-\infty<x<0} \frac{2}{(y-x)^{2}}|d x \wedge d y|=2 \log (z) .
$$

In the same way we compute:

$$
m(\{0<x<1 ; z<y<\infty\})=2 \log (z)-2 \log (z-1)
$$

The minimum of $m\left(Z_{z}\right)$ is achieved for $z=2, m\left(Z_{2}\right)=4 \log (2)$. This correspond to the "square" "torus" $T=\left\{e^{i k \pi / 2}\right\}$.

\subsection{Non-trivial spheres in $\mathcal{S}$, tangent spheres and twice tangent spheres.}

Observe first that a circle is intersected by a sphere in at most two points if the sphere does not contain the circle. Generically, a sphere can intersect a knot $K$ in 0,2 or any even number of points.

We remark that the volume of the set of spheres that intersect a given knot $K$ is infinite for any knot $K$. In order to have finite valued functional, we should get rid of the contribution of the set of "small spheres", which intersect $K$ in at most 2 points. 


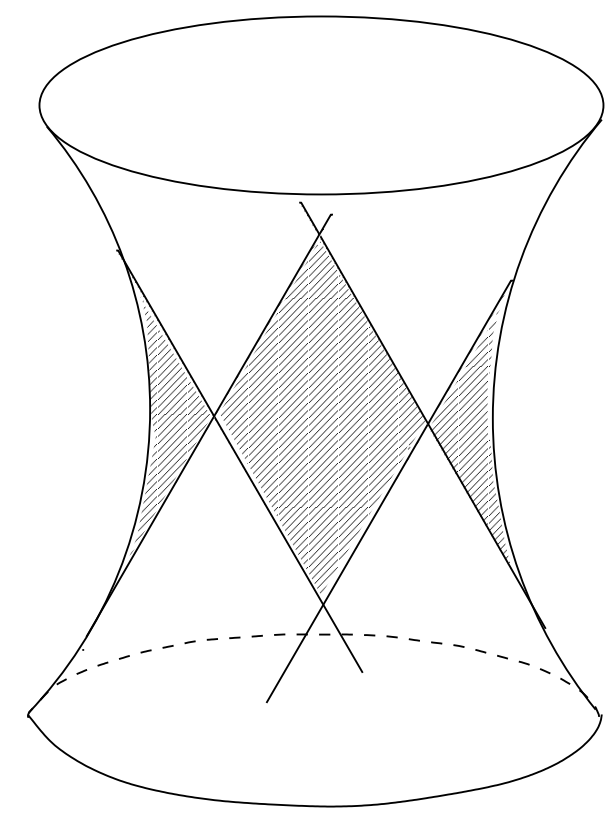

Figure 11: The set of the non-trivial 0-spheres

Definition 6.2.1 An oriented 2-sphere $\Sigma$ is called a non-trivial sphere for a knot $K$ if $\Sigma$ intersects $K$ in at least 4 points, where the number of the intersection points in $K \cap \Sigma$ is counted with multiplicity. An oriented 2-sphere $\Sigma$ with $\Sigma=\partial D^{3}$ is called a non-trivial sphere in the strict sense for a knot $K$ if $\operatorname{Int} D^{3} \cap K$ contains at least 2 connected components of $K \backslash \Sigma$.

Let $\overline{N T}(K) \subset \Lambda$ (or respectively, $N T(K) \subset \Lambda$ ) denote the set of the non-trivial spheres (or respectively, the non-trivial spheres in the strict sense).

If we fix a metric on the ambient sphere $S^{3}$, then for a constant $\alpha$ depending on the curve $K$, spheres of geodesical radius smaller than $\alpha$ can intersect $K$ in at most two points. This implies that the set $\overline{N T}(K)$ of non-trivial spheres is bounded in $\mathcal{S}$. It has therefore a finite volume.

Definition 6.2.2 Let mnts $_{1}(K)$ denote the volume of the set $\overline{N T}(K)$ of non-trivial spheres for a knot $K$.

The subset $\overline{N T}(K) \subset \mathcal{S}$ is, when $K$ is not a circle, a 4-dimensional region whose boundary consists of spheres which are tangent to the knot $K$. This boundary is not smooth. In particular, it has "corners" which are the transverse intersections of two folds of the set of the tangent spheres. Therefore the "corner" of $\partial \overline{N T}(K)$ consists of spheres which are tangent to the knot in two distinct points, that is, the twice tangent spheres. The closure of the boundary $\partial \overline{N T}(K)$ and the closure of its corner may have other singularities.

Definition 6.2.3 Let $T W T(K)$ denote the set of the twice tangent spheres and atwt $(K)$ its area. 
Remark: (1) Then the set $T W T(K)$ of the twice tangent spheres forms a surface of space type. Namely, the restriction of the Lorentz form to the tangent space at each regular point of this surface is positive definite. Therefore its area $\operatorname{atwt}(K)$ is a positive number, which is a conformal invariant of the knot $K$.

(2) Unlike $E^{(2)}$ or $E_{|\sin \theta|}$, atwt $(K)$ can not be expressed as an integral over $T^{2}$ of a function of the infinitesimal cross-ratio $\Omega_{C R}$ which does not contain its derivatives. This is because the local contribution of a neighborhood of a pair of points on $K$ to atwt $(K)$ depends on the curvature, whereas $\Omega_{C R}$ itself is independent of $f^{\prime \prime}$.

Question 6.2.4 Can atwt $(K)$ be expressed in terms of of the infinitesimal cross-ratio and its derivatives:

$$
\frac{e^{i \theta}}{|f(s)-f(t)|^{2}}, \frac{\partial}{\partial s}\left(\frac{e^{i \theta}}{|f(s)-f(t)|^{2}}\right), \text { etc.? }
$$

(3) The area $\operatorname{atwt}(K)$ is not an energy functional for knots.

it is enough to check that the contribution to $\operatorname{atwt}\left(K_{n}\right)$ of two ortogonal segments of length 1 , $I_{n} \subset K_{n}$ of middle point $p_{n}$, and $J_{n} \subset K_{n}$ of middle point $q_{n}$, such that $d\left(I_{n}, J_{n}\right)=d\left(p_{n}, q_{n}\right)=$ $1 / n$ does not blow up when $n$ goes to $\infty$. This contribution is bounded by the area of the spheres tangent to two orthogonal lines distant of 1 .

\subsection{The volume of the set of the non-trivial spheres.}

The measure $\operatorname{mnts}_{1}(K)$ of the set of the non-trivial spheres is not expressed as a "bilocal integral" like $E^{(2)}$ or $E_{|\sin \theta|}$ since it does not takes into account the number of intersection points of $K$ with spheres. In order to get such an integral, we have to count each sphere $\Sigma$ with multiplicity, which is a number of pairs of points in $\Sigma \cap K$ where the algebraic intersection number has the same sign.

Definition 6.3.1 The measure of acyclicity $m n t s(K)$ is defined by

$$
\operatorname{mnts}(K)=\int_{N T(K)} C_{2}^{n} d \Sigma, \text { where } n=n_{K}(\Sigma)=\frac{\sharp(\Sigma \cap K)}{2},
$$

where the number of the intersection points $\sharp(\Sigma \cap K)$ is counted with multiplicity, and $d \Sigma$ denotes the $\mathcal{G}$-invariant measure of $\Lambda$.

Another related functional of interest is the measure of the image of the 4-tuple map for a knot with multiplicity, which is given by

$$
m_{n t s}^{(4)}(K)=\int_{\operatorname{Conf}_{4}\left(S^{1}\right) \backslash \mathcal{C} c(K)} \psi_{K}{ }^{*} \omega_{\Lambda}=\int_{N T(K)} C_{4}^{2 n} d \Sigma,
$$

where $\omega_{\Lambda}$ is $\mathcal{G}_{+}$-invariant volume 4 -form of $\Lambda$. This definition of mnts $^{(4)}$ is based on the suggestion by J. Cantanella to the second author when he gave a talk on mnts $(K)$. It can not be expressed as a "bilocal integral". 
Claim 6.3.2 These three functionals, mnts $_{1}$, mnts, and mnts ${ }^{(4)}$ are conformally invariant.

Proof: Let $g \in \mathcal{G}$ be a conformal transformation. Then $g$ maps a non-trivial sphere $\Sigma$ of $K$ to a non-trivial sphere $g \cdot \Sigma$ of $g \cdot K$ preserving the number of the intersection points. Therefore $g(N T(K))=N T(g \cdot K)$ and $n_{K}(\Sigma)=n_{g \cdot K}(g \cdot \Sigma)$. Since the measure $d \Sigma$ is $\mathcal{G}$-invariant, i.e. $d \Sigma=d(g \cdot \Sigma)$, we have $\operatorname{mnts}(g \cdot K)=\operatorname{mnts}(K)$ etc.

As we mentioned before, the measure $\operatorname{mnts}_{1}(K)$ of the set $\overline{N T}(K)$ of the non-trivial spheres is finite for any smooth knot $K$ since $N T(K)$ is bounded in $\Lambda$. On the other hand, it is not easy to control the number of the intersection points of $K$ and a sphere $\Sigma$. We will show later at the end of the next subsection that the measure of acyclicity is finite for a smooth knot using formula 6.4.1.

\section{Question 6.3.3 Is mnts ${ }^{(4)}$ finite for any smooth knot?}

We conjecture that for any smooth knot $K$ there exists a natural number $n$ such that the measure of the set of spheres that intersect $K$ in at least $n$ points is 0 , and hence the answer to the above question is affirmative.

Remark: These three functionals, mnts ${ }_{1}$, mnts, and $m n t s^{(4)}$ are different. There holds $m_{n t s}(K) \leq m n t s(K) \leq$ mnts $^{(4)}(K)$. In fact, if an oriented 2-sphere $\Sigma$ has exactly 6 transversal intersection points with $K$, then $\Sigma$ is counted $1,3=C_{2}^{3}$, and $15=C_{4}^{6}$ times respectively in these functionals.

\subsection{The measure of acyclicity in terms of the infinitesimal cross- ratio.}

Assume that $K$ is oriented. Let $p \in K \cap \Sigma$ be a transversal intersection point. We say that $\Sigma$ intersects $K$ at p positively if the orientation of $K$ is "outward" at $p$ with respect to the orientation of $\Sigma$, namely, if the algebraic intersection number $K \cdot \Sigma$ is equal to +1 at $p$. We call $p$ a positive intersection point. Then the measure of acyclicity satisfies

$$
\operatorname{mnts}(K)=\int_{N T(K)} C_{2}^{n^{\prime}} \omega
$$

where $n^{\prime}$ is the number of positive intersection points of $K$ and $\Sigma$. We remark that the above definition does not depend on the choice of the orientation of a knot $K$.

Let $\left|N T_{K}^{(4)}\left(x, x^{\prime} ; y, y^{\prime}\right)\right|$ denote the volume of the set of the non-trivial spheres for $K$ which intersect $x x^{\prime}$ and $y y^{\prime}$ positively, where $x x^{\prime}$ denotes the curve segment of $K$ between $x$ and $x^{\prime}$ with the natural orientation derived from that of $K$. Put $\left|N T_{K}^{(4)}(d x, d y)\right|=\mid N T_{K}^{(4)}(x, x+$ $d x ; y, y+d y) \mid$. Let

$$
\left|n t_{K}^{(4)}(x, y)\right|=\lim _{\left|x-x^{\prime}\right|,\left|y-y^{\prime}\right| \rightarrow 0} \frac{\left|N T_{K}^{(4)}\left(x, x^{\prime} ; y, y^{\prime}\right)\right|}{\left|x-x^{\prime}\right|\left|y-y^{\prime}\right|} .
$$

Then the measure of acyclicity of $K$ is then equal to

$$
\operatorname{mnts}(K)=\lim _{\max \left|x_{i}-x_{i+1}\right| \rightarrow 0} \sum_{i<j}\left|N T_{K}^{(4)}\left(x_{i}, x_{i+1}, x_{j}, x_{j+1}\right)\right|=\frac{1}{2} \iint_{K \times K \backslash \triangle}\left|n t_{K}^{(4)}(x, y)\right| d x d y .
$$


Proposition 6.4.1 The measure of acyclicity is expressed as

$$
m n t s(K)=\frac{\pi}{4} \iint_{K \times K}(\sin \theta-\theta \cos \theta) \frac{d x d y}{|x-y|^{2}}
$$

where $\theta$ is the argument of cross-ratio.

The proof decomposes into the following two lemmas.

Lemma 6.4.2 There holds the dimension reduction formula:

$$
\left|N T_{K}^{(4)}(d x, d y)\right|=\frac{\pi}{2}\left|N T_{K}^{(3)}(d x, d y)\right|
$$

where $N T_{K}^{(3)}(d x, d y)$ denotes the the set of the non-trivial circles of the sphere $\Sigma(d x, d y)$ tangent to the knot at $x$ and $y$ which intersect both $d x$ and $d y$ positively. We denote by $\left|N T_{K}^{(3)}(d x, d y)\right|$ the volume of this set.

Proof: Since $\left|N T_{K}^{(4)}(d x, d y)\right|$ is conformally invariant, we may assume that $x, x+d x, y$ and $y+d y$ are in $\mathbb{R}^{2}=\{(X, Y, 0)\} \subset \mathbb{R}^{3}$ by a stereographic projection from the twice tangent sphere. Suppose the set of the non-trivial spheres or circles is parametrized by

$$
\begin{aligned}
C T R^{(3)}(d x, d y) & =\left\{(X, Y, r) \in \mathbb{R}_{+}^{3} \mid \begin{array}{l}
\exists C \in N T_{K}^{(3)}(d x, d y) \\
\text { with center }(X, Y) \text { and radius } r
\end{array}\right\} \\
C T R^{(4)}(d x, d y) & =\left\{(X, Y, Z, r) \in \mathbb{R}_{+}^{4} \mid \begin{array}{l}
\exists \Sigma \in N T_{K}^{(4)}(d x, d y) \\
\text { with center }(X, Y, Z) \text { and radius } r
\end{array}\right\} \\
& \cong\left\{\left(X, Y, Z, \sqrt{r^{2}+Z^{2}}\right) \mid(X, Y, r) \in C T R^{(3)}(d x, d y), Z \in \mathbb{R}\right\} .
\end{aligned}
$$

Then

$$
\begin{aligned}
\left|N T_{K}^{(4)}(d x, d y)\right| & =\int_{C T R^{(4)}(d x, d y)} \frac{1}{r^{4}} d X d Y d Z d r \\
& =\int_{C T R^{(3)}(d x, d y)}\left(\int_{-\infty}^{\infty} \frac{1}{\left(r^{2}+Z^{2}\right)^{2}} d Z\right) d X d Y d r \\
& =\frac{\pi}{2} \int_{C T R^{(3)}(d x, d y)} \frac{1}{r^{3}} d X d Y d r=\frac{\pi}{2}\left|N T_{K}^{(3)}(d x, d y)\right|
\end{aligned}
$$

In what follows we calculate $\left|N T_{K}^{(3)}(d x, d y)\right|$ in the case when $\theta$ satisfies $0 \leq \theta \leq \frac{\pi}{2}$. Similar calculation works for $\frac{\pi}{2} \leq \theta \leq \pi$.

We may assume that

$$
x=\infty, x+d x=(0,0), y=(1,0), \text { and } y+d y=(1+a, b) \quad(b \geq 0)
$$


by a suitable orientation preserving conformal transformation. Then, as the cross-ratio is preserved,

$$
a=\cos \theta \frac{|d x||d y|}{|x-y|^{2}}, b=\sin \theta \frac{|d x||d y|}{|x-y|^{2}} .
$$

Then $C T R^{(3)}(d x, d y)$ is given by

$$
C T R^{(3)}(d x, d y)=\left\{\begin{array}{l|l}
(X, Y, r) \in \mathbb{R}_{+}^{3} & \begin{array}{l}
X^{2}+Y^{2}<r^{2} \\
(X-1)^{2}+Y^{2}>r^{2} \\
(X-(1+a))^{2}+(Y-b)^{2}<r^{2}
\end{array}
\end{array}\right\}
$$

Let $\mathcal{N}\left(r_{0}\right)$ be the intersection of $C T R^{(3)}(d x, d y)$ and the level plane defined by $\left\{r=r_{0}\right\}$. Then

$$
\left|N T_{K}^{(3)}(d x, d y)\right|=\int_{0}^{\infty} \frac{1}{r^{3}} \operatorname{area}(\mathcal{N}(r)) d r .
$$

Let $C_{i}(r)$ be circles defined by

$$
\begin{aligned}
& C_{1}(r)=\left\{(X, Y) \mid X^{2}+Y^{2}=r^{2}\right\}, \\
& C_{2}(r)=\left\{(X, Y) \mid(X-1)^{2}+Y^{2}=r^{2}\right\}, \\
& C_{3}(r)=\left\{(X, Y) \mid(X-(1+a))^{2}+(Y-b)^{2}=r^{2}\right\} .
\end{aligned}
$$

Let $P_{i j}(r)=\left(X_{i j}(r), Y_{i j}(r)\right)(i \neq j)$ be one of the two intersection points of $C_{i}(r) \cap C_{j}(r)$ (we chose the one with bigger $y$-coordinate as is shown in Figure 12).

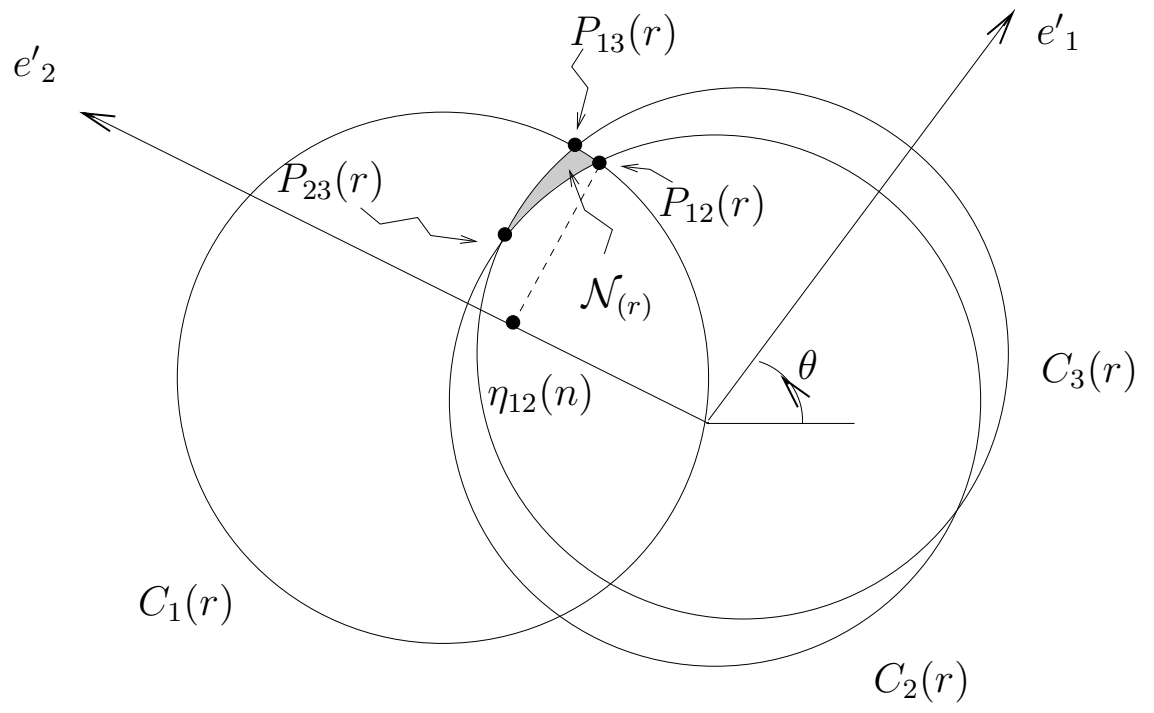

Figure 12: $\mathcal{N}(r)$ and $P_{i j}(r)$ 


$$
\begin{aligned}
& P_{12}(r)=\left(\frac{1}{2}, \sqrt{r^{2}-\frac{1}{4}}\right), \\
& P_{13}(r)=\left(\frac{1+a}{2}-b \sqrt{\frac{r^{2}}{(1+a)^{2}+b^{2}}-\frac{1}{4}}, \frac{b}{2}+(1+a) \sqrt{\frac{r^{2}}{(1+a)^{2}+b^{2}}-\frac{1}{4}}\right), \\
& P_{23}(r)=\left(1+\frac{a}{2}-\frac{b}{\sqrt{a^{2}+b^{2}}} \sqrt{r^{2}-\frac{a^{2}+b^{2}}{4}}, \frac{b}{2}+\frac{a}{\sqrt{a^{2}+b^{2}}} \sqrt{r^{2}-\frac{a^{2}+b^{2}}{4}}\right) .
\end{aligned}
$$

Let $\left(\xi_{i j}(r), \eta_{i j}(r)\right)$ be the coordinate of $P_{i j}(r)$ with respect to the frame $\left((1,0), e_{1}^{\prime}, e_{2}^{\prime}\right)$ where $e_{1}^{\prime}=(\cos \theta, \sin \theta)$ and $e_{2}^{\prime}=(-\sin \theta, \cos \theta)$.

Lemma 6.4.3 There holds

$$
\begin{aligned}
\left|N T_{K}^{(3)}(d x, d y)\right| & =\int_{\frac{1}{2 \sin \theta}}^{\infty} \frac{1}{r^{3}} \cdot \sqrt{a^{2}+b^{2}}\left(\eta_{23}(r)-\eta_{12}(r)\right) d r+o\left(\sqrt{a^{2}+b^{2}}\right) \\
& =\left(\int_{\frac{1}{2 \sin \theta}}^{\infty} \frac{\eta_{23}(r)-\eta_{12}(r)}{r^{3}} d r\right) \frac{|d x||d y|}{|x-y|^{2}}+o\left(\frac{|d x||d y|}{|x-y|^{2}}\right) .
\end{aligned}
$$

\section{Proof:}

We first remark that $\mathcal{N}(r)$ is not empty if and only if $X_{12}(r) \geq X_{23}(r)$, which is equivalent to $1-r \sin \theta \leq \frac{1}{2}$ when $a, b \ll 1$.

The second equality is a consequence of

$$
\sqrt{a^{2}+b^{2}}=\frac{|d x||d y|}{|x-y|^{2}}
$$

Let $\mathcal{P}(r) \subset \mathbb{R}^{2}$ be a subset which is swept by the arc in $C_{2}(r)$ between $P_{23}(r)$ and $P_{12}(r)$ when it moves parallel by $\sqrt{a^{2}+b^{2}}$ in the direction $e_{1}^{\prime}$. Let $M_{1}(r)$ be a curved triangle bounded by $C_{1}(r), C_{3}(r)$ and the half-line startin from $P_{12}(r)$ in the direction $e_{1}^{\prime}$, and let $M_{2}(r)$ be a subset bounded by $C_{3}(r)$ and the half-line startin from $P_{23}(r)$ in the direction $e_{1}^{\prime}$ as in Figure 13.

Then $\mathcal{N}(r)=\left(\mathcal{P}(r) \cup M_{2}(r)\right) \backslash M_{1}(r)$. We have only to show that

$$
\int_{\frac{1}{2 \sin \theta}}^{\infty} \frac{1}{r^{3}} \cdot \operatorname{area}\left(M_{i}(r)\right) d r=o\left(\sqrt{a^{2}+b^{2}}\right) \quad(i=1,2) .
$$

Since area $\left(M_{1}(r)\right) \leq a^{2}+b^{2}$, (8) holds for $i=1$. On the other hand 


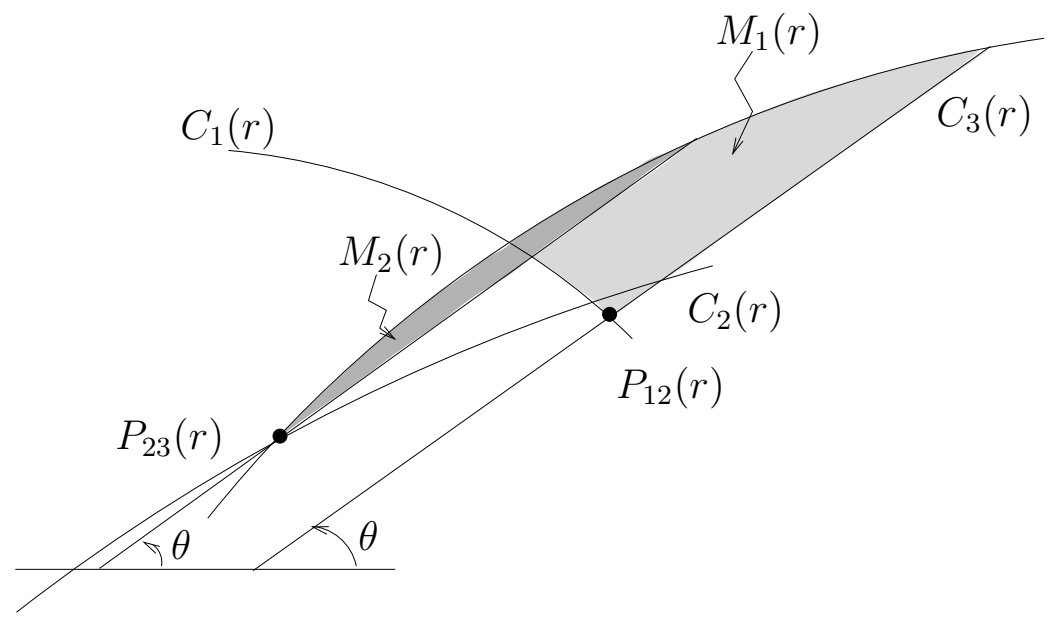

Figure 13: The regions $M_{1}(r)$ and $M_{2}(r)$

$$
\begin{aligned}
& \operatorname{area}\left(M_{2}(r)\right)=\frac{1}{2} \sqrt{a^{2}+b^{2}}\left(\eta_{13}(r)-\eta_{12}(r)\right)+o\left(\sqrt{a^{2}+b^{2}}\right) \\
& =\frac{1}{2} \sqrt{a^{2}+b^{2}}\left\{\sin \theta \cdot\left(X_{12}(r)-X_{13}(r)\right)-\cos \theta \cdot\left(Y_{12}(r)-Y_{13}(r)\right)\right\}+o\left(\sqrt{a^{2}+b^{2}}\right) .
\end{aligned}
$$

Since

$$
X_{12}(r)-X_{13}(r)=\sqrt{a^{2}+b^{2}}\left(-\frac{\cos \theta}{2}+\sin \theta \sqrt{\frac{r^{2}}{(1+a)^{2}+b^{2}}-\frac{1}{4}}\right),
$$

we have

$$
\begin{aligned}
& \int_{\frac{1}{2 \sin \theta}}^{\infty} \frac{1}{r^{3}} \cdot \frac{1}{2} \sqrt{a^{2}+b^{2}} \cdot \sin \theta \cdot\left(X_{12}(r)-X_{13}(r)\right) d r \\
& \leq \frac{\sin \theta}{2}\left(a^{2}+b^{2}\right) \int_{\frac{1}{2 \sin \theta}}^{\infty}\left(-\frac{\cos \theta}{2 r^{3}}+\frac{\sin \theta}{r^{2}}\right) d r=O\left(a^{2}+b^{2}\right) .
\end{aligned}
$$

On the other hand since

$$
\begin{aligned}
Y_{13}(r)-Y_{12}(r) & =\frac{b}{2}+a \sqrt{\frac{r^{2}}{(1+a)^{2}+b^{2}}-\frac{1}{4}}+\sqrt{\frac{r^{2}}{(1+a)^{2}+b^{2}}-\frac{1}{4}}-\sqrt{r^{2}-\frac{1}{4}} \\
& =\frac{b}{2}+a \sqrt{\frac{r^{2}}{(1+a)^{2}+b^{2}}-\frac{1}{4}}-\frac{\frac{2 a+a^{2}+b^{2}}{(1+a)^{2}+b^{2}} r^{2}}{\sqrt{r^{2}-\frac{1}{4}}+\sqrt{\frac{r^{2}}{(1+a)^{2}+b^{2}}-\frac{1}{4}}}
\end{aligned}
$$


we have

$$
\begin{aligned}
& \int_{\frac{1}{2 \sin \theta}}^{\infty} \frac{1}{r^{3}} \cdot \frac{1}{2} \sqrt{a^{2}+b^{2}} \cdot \cos \theta \cdot\left(Y_{13}(r)-Y_{12}(r)\right) d r \\
& \leq \frac{\cos \theta}{2}\left(a^{2}+b^{2}\right) \int_{\frac{1}{2 \sin \theta}}^{\infty}\left(\frac{\sin \theta}{2 r^{3}}+\frac{\cos \theta}{r^{2}}+\frac{\cos \theta}{r \sqrt{r^{2}-\frac{1}{4}}}\right) d r+o\left(\sqrt{a^{2}+b^{2}}\right)
\end{aligned}
$$

as $\sqrt{r^{2}-\frac{1}{4}} \geq r \cos \theta$ when $r \geq \frac{1}{2 \sin \theta}$, the right hand side satisfies

$$
\leq \frac{\cos \theta}{2}\left(a^{2}+b^{2}\right) \int_{\frac{1}{2 \sin \theta}}^{\infty}\left(\frac{\sin \theta}{2 r^{3}}+\frac{1+\cos \theta}{r^{2}}\right) d r+o\left(\sqrt{a^{2}+b^{2}}\right)=O\left(a^{2}+b^{2}\right) .
$$

This completes the proof of Lemma 6.4.3.

Proof of Proposition 6.4.1; Since

$$
\begin{aligned}
\eta_{23}(r)-\eta_{12}(r) & =\sin \theta\left(X_{12}(r)-X_{23}(r)\right)-\cos \theta\left(Y_{12}(r)-Y_{23}(r)\right) \\
& =\sqrt{r^{2}-\frac{a^{2}+b^{2}}{4}}-\frac{\sin \theta}{2}-\cos \theta \sqrt{r^{2}-\frac{1}{4}}
\end{aligned}
$$

we have

$$
\begin{aligned}
& \int_{\frac{1}{2 \sin \theta}}^{\infty} \frac{\eta_{23}(r)-\eta_{12}(r)}{r^{3}} d r=\int_{\frac{1}{2 \sin \theta}}^{\infty} \frac{1}{r^{3}}\left(r-\frac{\sin \theta}{2}-\cos \theta \sqrt{r^{2}-\frac{1}{4}}\right) d r+o\left(\sqrt{a^{2}+b^{2}}\right) \\
& \quad=2 \int_{0}^{\sin \theta}\left(1-u \sin \theta-\cos \theta \sqrt{1-u^{2}}\right) d u+o\left(\sqrt{a^{2}+b^{2}}\right) \quad\left(u=\frac{1}{2 r}\right) \\
&=\sin \theta-\theta \cos \theta+o\left(\sqrt{a^{2}+b^{2}}\right),
\end{aligned}
$$

which, combined with Lemma 6.4.3. completes the proof of Proposition 6.4.1.

Lemma 5.1.2 implies that the integrand $(\sin \theta-\theta \cos \theta) /|x-y|^{2}$ of the measure of acyclicity is 0 at the diagonal set. Therefore, the measure of acyclicity is finite for any knot of class $C^{4}$. Lemma 5.1 .2 also implies that $E^{(2)}, E_{|\sin \theta|}$, and mnts are independent.

We remark that Claim 4.1.4 implies that the measure of acyclicity of $K$, and hence $m n t s{ }^{(4)}(K)$, is equal to 0 if and only if $K$ is the standard circle.

\section{Non-trivial zones.}

\subsection{Non-trivial zones.}

A circle is generically intersected by a sphere in 0 or two point. It is a necessary and sufficient condition for a knot to be the circle. A curve which is not a circle should therefore admit spheres intersecting it transversally in at least 4 points. Thickening such a non-trivial sphere, we get a region bounded by two disjoint spheres which is crossed by at least 4 strands. 
Definition 7.1.1 (1) A zone $Z$ is a region of $S^{3}$ or $\mathbb{R}^{3}$ diffeomorphic to $S^{2} \times[0,1]$ bounded by two disjoint spheres.

(2) Let $Z$ be a zone with $\partial Z=S_{1} \cup S_{2}$ and $T: \mathbb{R}^{3}$ (or $\left.S^{3}\right) \rightarrow \mathbb{R}^{3}$ (or $\mathbb{R}^{3} \cup\{\infty\}$ respectively) be a conformal transformation which maps the two boundary spheres of $Z$ into a concentric position. Then the modulus $\rho(Z)>0$ of the zone $Z$ is $\rho(Z)=\left|\log \frac{R_{1}}{R_{2}}\right|$ where $R_{1}$ and $R_{2}$ are the radii of the two concentric boundary spheres of $T(Z)$.

(3) Instead of using a particular conformal transformation $T$, one can use the Lorentzian modulus $\lambda(Z)=\left|L\left(S_{1}, S_{2}\right)\right|$ of a zone $Z$ with $\partial Z=S_{1} \cup S_{2}$, where $S_{1}$ and $S_{2}$ are considered $^{8}$ as points in $\Lambda$.

\section{Remark:}

(1) A zone $Z$ is uniquely determined by its modulus up to a motion by a conformal transformation.

(2) The modulus $\rho(Z)$ is an increasing function of the Lorentzian modulus $\lambda(Z)$ with:

$$
\rho=\log \left(\lambda+\sqrt{\lambda^{2}-1}\right) \text { and } \lambda=C h \rho .
$$

In fact, one can assume, after a conformal transformation, that $S_{1}$ is an equator 2-sphere of $S^{3}$ and $S_{2}$ is a parallel 2-sphere to $S_{1}$. Then the Poncelet pencil in $\Lambda$ generated by $S_{1}$ and $S_{2}$, which is a hyperbola in the 2-plane in $\mathbb{R}^{5}$ generated by $S_{1}$ and $S_{2}$, can be parametrized by $(C h t, S h t)$, where $t=0$ corresponds to $S_{1}$. The restriction of $L$ to this Poncelet pencil is a quadratic form of type $(1,1)$. Let $t_{0}$ be the parameter for $S_{2}$. Then $\rho(Z)=t_{0}$ whereas $\lambda(Z)=C h t_{0}$.

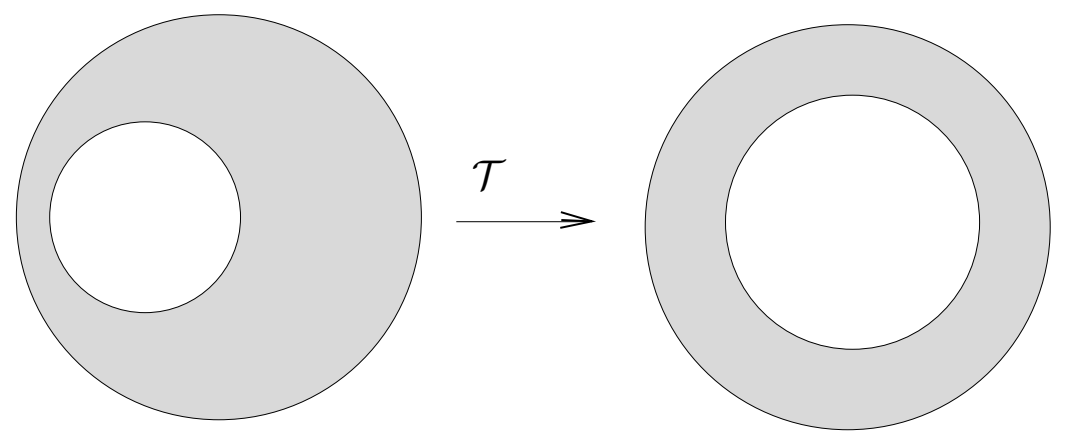

Figure 14: A zone and its privileged representative

It can also be computed from the cross ratio of the four points intersections of the two spheres with a circle containing the two base points of the pencil generated by the two spheres. Remark: If the zones $Z_{1}$ and $Z_{2}$ satisfy $Z_{1} \subset Z_{2}$, one has $\rho\left(Z_{2}\right) \geq \rho\left(Z_{1}\right)$.

Definition 7.1.2 Let $K$ be a knot and $Z$ be a zone with $\partial Z=S_{1} \cup S_{2}$.

(1)A strand is an arc of $K$ that is contained in the zone $Z$ and whose end points are on $\partial Z$. A small strand (of $K$ in $Z$ ) is a closure of a connected component of the intersection of the

\footnotetext{
${ }^{8} S_{1}$ and $-S_{2}$ are endowed with the orientation as the boundary of $Z$
} 
interior of $Z$ with $K$ whose two end points lie on the same boundary sphere of $Z$. A degenerate small strand is a closed connected component of the intersection of $K$ with $\partial Z$.

(2) A cross-strand is a strand of $K$ with one end point contained in $S_{1}$ and another in $S_{2}$. A minimal cross-strand is a cross-strand whose intersection with the interior of $Z$ is connected. If a cross-strand has a common end point with a small strand, one can connect them to obtain a longer cross-strand.

(3) A zone will be called non-trivial for a knot $K$ if its intersection with $K$ contains at least 4 closed cross-strands of the knot $K$ with disjoint interiors.

The existence of spheres intersecting a curve in at least four points transversally implies the existence of non-trivial zones. In particular such non-trivial zones exist for a non-trivial knot $K$.

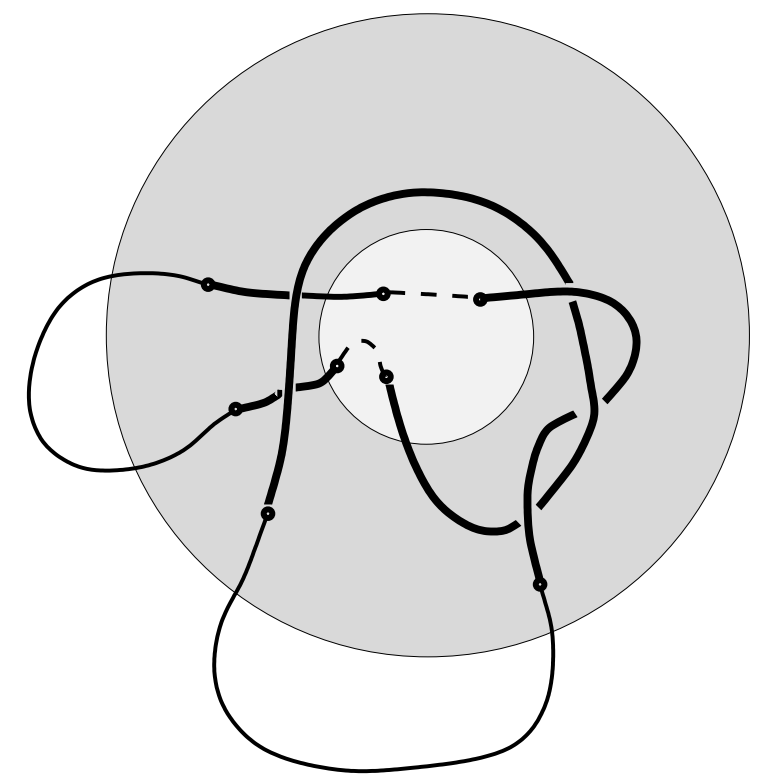

Figure 15: Non-trivial zone

Definition 7.1.3 The modulus $\rho$ of a knot $K$ is the supremum of the modulus of a non-trivial zone for $K$.

$$
\rho=\sup _{A: \text { non-trivial zone }} \rho(A)
$$

Definition 7.1.4 A non-trivial zone for a knot $K$ is called maximal if it has the maximum modulus among the set of non-trivial zones for $K$.

Remark: There exists a maximal non-trivial zone for any non-trivial knot $K$ since the set of pairs of boundary spheres of non-trivial zones is a non-empty compact subset of $\Lambda \times \Lambda$, as a "small" sphere intersects the knot in two points at most, and in only one point if it is tangent to $K$.

Let us now look for properties of maximal non-trivial zones. 
Proposition 7.1.5 Let $Z$ be a maximal non-trivial zone for $K$ with $\partial Z=S_{1} \cup S_{2}$. appying a suitable conformal transformation, we can suppose that $S_{1}$ and $S_{2}$ are spheres of $\mathbb{R}^{3}$ centered at the origin. Then it satisfies the following.

$K$ is contained in $Z$.

Proposition 7.1.6 Under the same assumptions as above,

(1) $K \cap S_{1}$ (or $K \cap S_{2}$ ) consists of two points where $K$ is tangent to $S_{1}$ (or $S_{2}$, respectively).

(2) The two tangent points of $K \cap S_{1}$ (or $K \cap S_{2}$ ) are antipodal.

let us prove first the proposition 7.1 .5

Proof: The knot $K$ is a finite union of open minimal cross-strands: $m_{1}, \ldots, m_{n}$, and arcs or points contained in $\partial Z: c_{1}, \ldots, c_{n} ; K=m_{1} \cup c_{1} \cup m_{2} \cup c_{2} \ldots \cup m_{n} \cup c_{n}$ in cyclic order on $K$. Each $\operatorname{arc} c_{i}$ meets either $S_{1}$ or $S_{2}$ but cannot meet both.

Suppose that one of these $\operatorname{arcs}$, say $c_{1}$, meets $S_{1}$ and exits $Z$. At least two other points or $\operatorname{arcs} c_{n}$ and $c_{2}$ meet $S_{2}$. The $\operatorname{arcs} m_{3}$ and $m_{n} n \geq 4$ exist. The difference $K \backslash\left(m_{n} \cup c_{n} \cup m_{1} \cup\right.$ $c_{1} \cup m_{2} \cup c_{2} \cup m_{3}$ ) is a closed arc containing (at least) a point $A \in S_{1}$. Moving $S_{1}$ in the pencil of spheres tangent to $S_{1}$ at $A$, we can enlarge the zone $Z$ keeping minimal strands containing $m_{1}$ and $m_{2}$.

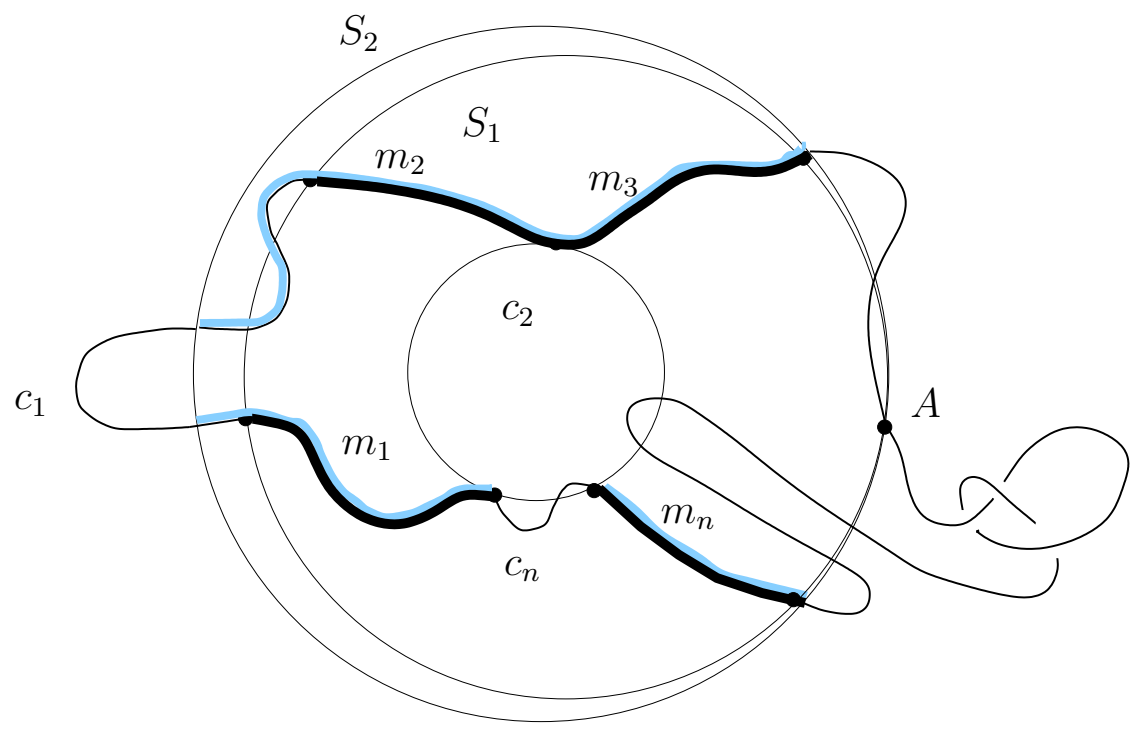

Figure 16: Increasing the modulus of the zone $Z$

Corollary 7.1.7 If a non-trivial zone $Z$ with $\partial Z=S_{1} \cup S_{2}$ is maximal, then the points $c_{i}$ or both endpoints of the arcs $c_{i}$ are alternatively contained in $S_{1}$ and in $S_{2}$. Therefore at least two are contained in or touch each of spheres $S_{1}$ and $S_{2}$

To prove the proposition 7.1.6 we will show that, if we can find on the sphere $S_{1}$ two points $x_{1} \in c_{i}, x_{3} \in c_{j}$ belonging to two different $\operatorname{arcs} c_{i} \neq c_{j}$ which are not antipodal, or in the sphere 
$S_{2}$ two points $x_{2} \in c_{k}, x_{4} \in c_{l}$ belongings to two different arcs $c_{k} \neq c_{l}$ which are not antipodal, then the zone $Z$ is not maximal. The numeration of the four points can be chosen such that they are in cyclic order on $K$.

\subsubsection{The modulus of 4 points}

For a moment, let us consider only the four points $x_{1}, x_{2}, x_{3}, x_{4}$ and forget the rest of the knot.

Definition 7.1.8 Let $x_{1}, x_{2}, x_{3}$, and $x_{4}$ be ordered four points in $\mathbb{R}^{3}$ (or $S^{3}$ ).

(1) A zone $Z$ with $\partial Z=S_{1} \cup S_{2}$ is called to be separating if $x_{1}$ and $x_{3}$ are contained in one of the connected component of $\mathbb{R}^{3} \backslash \operatorname{Int} Z$ (or $S^{3} \backslash \operatorname{Int} Z$ ) and $x_{2}$ and $x_{4}$ in another component.

(2) The maximal modulus $\rho\left(x_{1}, x_{2}, x_{3}, x_{4}\right)$ of the ordered four points $\left(x_{1}, x_{2}, x_{3}, x_{4}\right)$ is the supremum of the moduli $\rho(Z)$ of separating zones $Z$ with $\partial Z=S_{1} \cup S_{2}\left(\right.$ when $\left.S_{1}=S_{2}, \rho=0\right)$.

(3) A separating zone that attains the maximal modulus will be called the maximal separating zone of the four point.

(4) We can also consider $L\left(S_{1}, S_{2}\right)$, where with $\partial Z=S_{1} \cup S_{2}$, and define $\lambda\left(x_{1}, x_{2}, x_{3}, x_{4}\right)$ to be the value of $L\left(S_{1}, S_{2}\right)$ for a maximal separating zone (when $S_{1}=S_{2}, \lambda=1$ ).

We remark that if $Z$ is a maximal separating zone of the four points, then one of its boundary sphere should contain both $x_{1}$ and $x_{3}$, and the other $x_{2}$ and $x_{4}$.

Lemma 7.1.9 (1) There are no separating zone, i.e. $\rho=0 ; \mu_{L}=1$ if and only if $x_{i}$ 's are concircular in such a way that $x_{1}$ and $x_{3}$ are not adjacent, i.e. when the the cross-ratio $\left(x_{2}, x_{3} ; x_{1}, x_{4}\right)$ is a real number between 0 and 1 .

(2) Let $Z$ be a maximal separating zone for $\left(x_{1}, x_{2}, x_{3}, x_{4}\right)$ with $\partial Z=S_{1} \cup S_{2}$. Suppose we transform the picture by a conformal map so that the images of $S_{1}$ and $S_{2}$ are concentric. Then on the new picture, $x_{1}$ and $x_{3}$ are antipodal in one of the boundary spheres of $Z$, and $x_{2}$ and $x_{4}$ are antipodal in the other boundary sphere.

(3) When separating zones exist, the maximal separating zone $Z$ is unique.

\section{Remark:}

(1) The condition of being antipodal does not seem to be conformally invariant.

The condition can be rephrased as follows: the two points $x_{1}$ and $x_{3}$ have to belong to a circle containing the two limit points of the pencil generated by the two boundary spheres of the zone; the two points $x_{2}$ and $x_{4}$ satisfy a similar condition (the circle containing $x_{2}$ and $x_{4}$ belong to the same pencil as the circle containing $x_{1}$ and $x_{3}$ ). The conformal transformation such that the images of $S_{1}$ and $S_{2}$ are antipodal has to send the limit poins of the pencil generated by the two boundary spheres of the zone to 0 and $\infty$; circles through the limit points become lines through the origin.

(2) The proof of statement (3) will be given in the appendix.

(3) The statement (2) and (3)of the lemma also holds when the four points are concircular and $\left(x_{1}, x_{3}\right)$ are consecutive (and also $\left(x_{2}, x_{4}\right)$ ). 

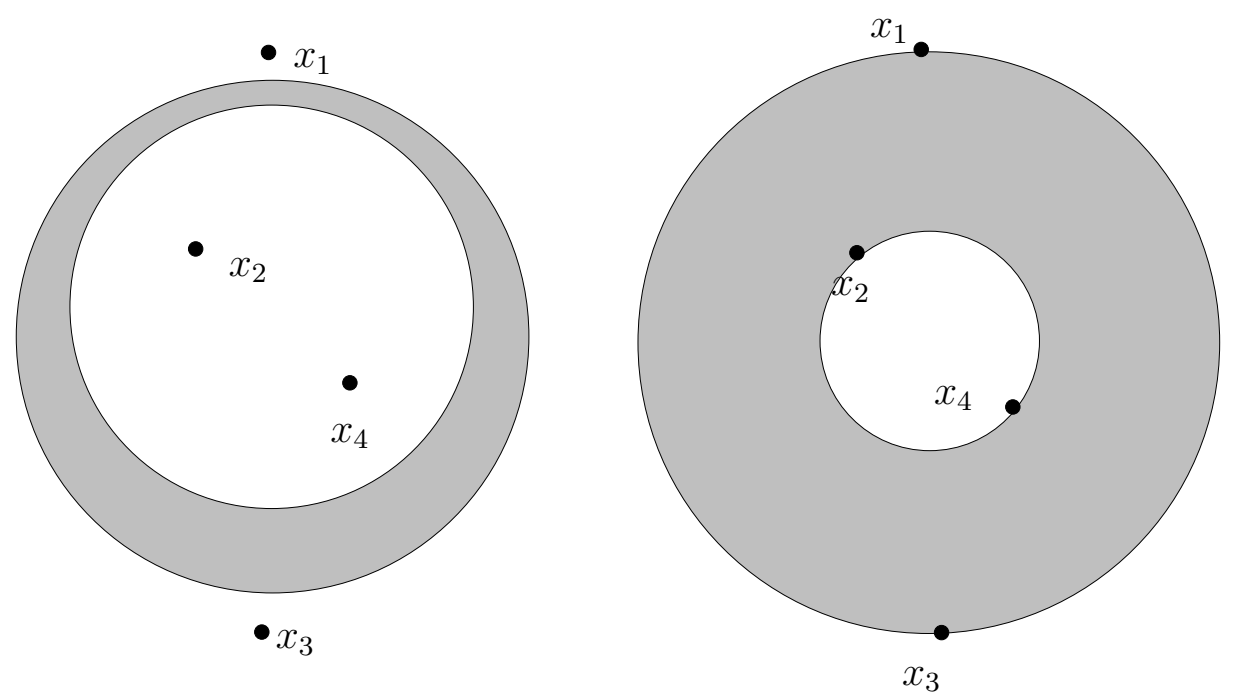

Figure 17: Maximal modulus for four points

The proof is the consequence of the following sublemma ${ }^{9}$ :

Sublemma 7.1.10 Let $\mathcal{P}$ be a Poncelet pencil of spheres; it is the intersection of $\Lambda$ with a plane $P$. Let $\mathcal{B}$ be the two dimensional linear family of spheres $\mathcal{B}=B \cap \Lambda$ where $B=P^{\perp}$. At a point $\sigma \in \mathcal{P}$ the 3-plane orthogonal to $\mathcal{P}$ is generated by the vectors tangent at $\sigma$ to the pencils of spheres with base circle a circle of the form $\left(\sigma^{\perp} \cap S_{\infty}\right) \cap\left(b^{\perp} \cap S_{\infty}(b \in \mathcal{B})\right.$.

\section{Proof of the sublemma 7.1.10,}

The intersection $\mathcal{B}=B \cap \Lambda$ is the set of spheres containing the two limit points of the Poncelet pencil $\mathcal{P}$. Let $F=B \oplus \mathbb{R} \cdot \sigma$. The tangent vectors to $F \cap \Lambda$ are in $F \cap T_{\sigma} \Lambda=F \cap \sigma^{\perp}=B$; therefore the tangent space at $\sigma$ to $F \cap \Lambda$ is an affine 3 -space parallel to $B$. Hence it is orthogonal to $\mathcal{P}$.

The tangent at $\sigma$ to $\mathcal{P}$ is time-like. The vector space generated by $\sigma$ and a point $b \in \mathcal{B}$ is space like. It intersects $\Lambda$ in a closed geodesic. The tangent line at $\sigma$ to this geodesic is parallel to $b$. This closed geodesic is a pencil of spheres with base circle $\left(\sigma^{\perp} \cap S_{\infty}^{3}\right) \cap\left(b^{\perp} \cap S_{\infty}^{3}\right)$ (see fig (7.1.1).

Proof of Lemma 7.1.9]: The last sublemma implies the lemma as, if two points contained in $S_{1}$ are not opposite, there exists a non geodesic circle $\gamma$ containing them.

Let us call $\mathcal{B}_{\gamma}$ the pencil with base circle $\gamma$. Consider the Poncelet pencil $\mathcal{P}$ generated by $S_{1}$ and $S_{2}$. The Lorentz modulus $\lambda$ of the region bounded by the disjoint spheres $S_{2}$ and $\sigma \in \mathcal{P}$ is just $\left|L\left(S_{2}, \sigma\right)\right|$. Let us call this function $\varphi(\sigma)$. The connected component of its level through $S_{1}$ is therefore the hypersurface of equation $L\left(S_{2}, \tau\right)=L\left(S_{2}, S_{1}\right)$. Its (affine) tangent plane at $S_{1}$ has the equation: $L\left(S_{2}, v-S_{1}\right)=0$. The set of tangent vectors to this level is $B=P^{\perp}$ (where $P$ is the plane such that $\mathcal{P}=P \cap \Lambda$ ).

Before finishing the proof, we transform the picture by a conformal map sending the limit points of $\mathcal{P}$ to 0 and $\infty$. Therefore the spheres $S_{1}$ and $S_{2}$ are concentric.

\footnotetext{
${ }^{9}$ We state other proofs in the subsection 8.1
} 


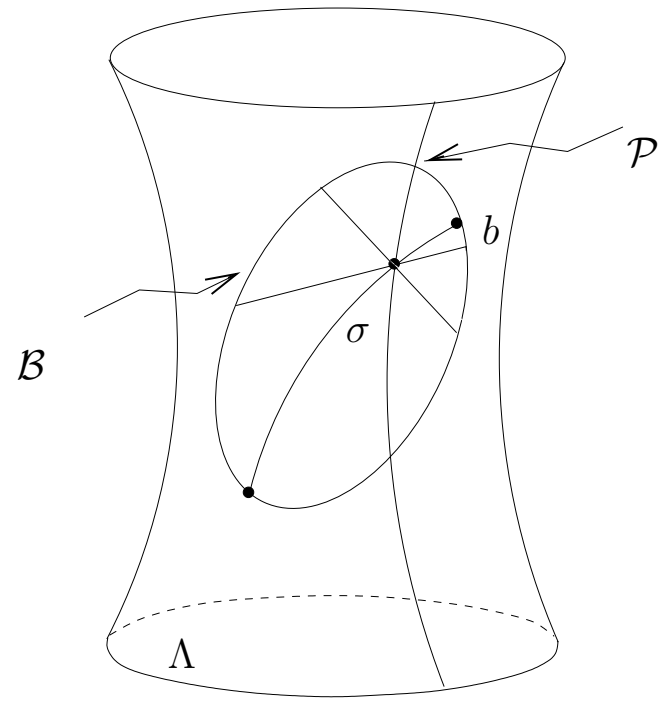

(a)

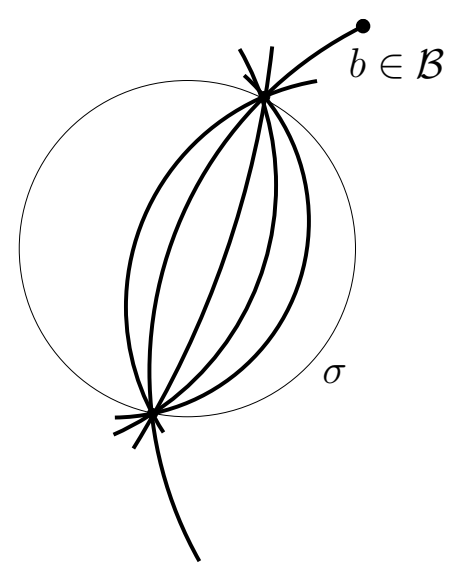

(b)

Figure 18: The 3-plane in $T_{\sigma} \Lambda$ orthogonal at $\sigma$ to $T_{\sigma} \mathcal{P}$

Spheres $b \in \mathcal{B}$ (notation of the sublemma) appear as planes through the origin. Therefore the circle basis of the pencil generated by $S_{1}$ and $b$ is a geodesic circle on $S_{1}$.

It is equivalent to say that a vector $w \in T_{S_{1}} \Lambda$ is not orthogonal to $\mathcal{P}$ or to say that the vector $w$ is tangent at $S_{1}$ to a pencil with base circle which is not a geodesic circle of $S_{1}$.

Therefore, for $w$ a vector tangent to $\mathcal{B}_{\gamma}$ at $S_{1}$ we have $L\left(S_{2}, w\right) \neq 0$. This implies that the derivative of the Lorentzian modulus $\lambda$ of the region between $S_{2}$ and a sphere $\tau \in \mathcal{B}$ is not zero in $S_{1}$, allowing to increase the modulus of the zone keeping the four points $x_{1}, x_{2}, x_{3}$ and $x_{4}$ in the boundary spheres.

What we did for the Lorentzian modulus $\lambda$ is enough to prove the same result for the modulus $\rho$, as the correspondance between $\lambda$ and $\rho$ is a diffeomorphism, see the formula (9).

\subsubsection{Knots of small moduli}

The goal of this section is to prove the:

Theorem 7.1.11 There exists a constant $a>0$ such that if a knot $K$ is a non-trivial knot, then its modulus is larger than a.

Let us now start with a curve $K$ of modulus $\rho$. Let us consider a stereographic projection to $\mathbb{R}^{3}$ such that the two boundary spheres of a maximal zone $Z$ are concentric, with center the origin. We may imagine a very thin knitted curve contained in a zone of small modulus. In order to prove knot is trivial, we need to use the condition that the maximal modulus is very small to control the knot at all scales.

We remark that the modulus $\rho(K)$ of a knot $K$ is larger than the maximal modulus $\mu\left(x_{1}, x_{2}, x_{3}, x_{4}\right)$ for any four points on the knot chosen in that cyclic order, since any separating zone of $\left(x_{1}, x_{2}, x_{3}, x_{4}\right)$ is a non-trivial zone for $K$.

Fixing three points $x_{1}, x_{2}, x_{3}$ defines a function $\mu_{x_{1}, x_{2}, x_{3}}$ by: 


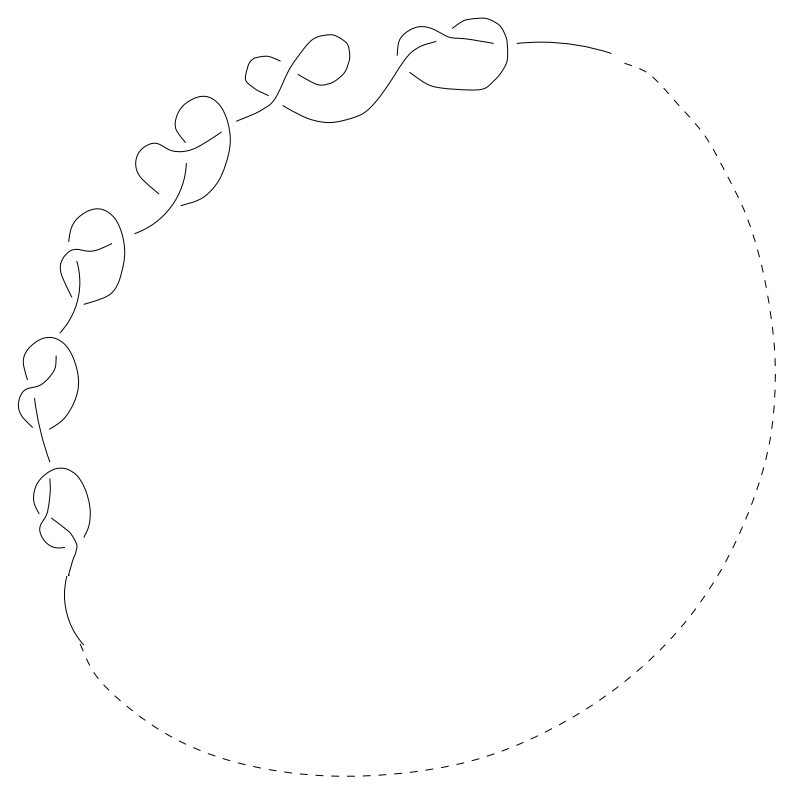

Figure 19: Knitted curve

$\mu_{x_{1}, x_{2}, x_{3}}(x)=\mu\left(x_{1}, x_{2}, x_{3}, x\right)$. It is a continuous function, the zero level of which is the arc $\gamma$ of the circle trough $\left(x_{1}, x_{2}, x_{3}\right)$ which does not contain the point $x_{2}$. Moreover, given a small value $\epsilon$ of $\mu_{x_{1}, x_{2}, x_{3}}$, if the sphere $S^{2}$ is endowed with a metric such that the length of the circle through $x_{1}, x_{2}, x_{3}$ is of the order of 1 , then the distance from the level $\mu_{x_{1}, x_{2}, x_{3}}=\epsilon$ to the circle $\gamma$ satisfies:

$$
\forall x \in\left\{\mu_{x_{1}, x_{2}, x_{3}}=\epsilon\right\} ; a \cdot \operatorname{length}(\gamma)<d(x, \gamma)<A \cdot \operatorname{length}(\gamma) .
$$

Let us now suppose that the curve $K$ has a small modulus, say less than $\frac{1}{10000}$, and chose a stereographic projection of $S^{3}$ on $\mathbb{R}^{3}$ such that the two boundary spheres $S_{1}$ and $S_{2}$ of a maximal zone $Z$ for $K$ are concentric with center the origin (the north pole of the projection is one of the limit points of the pencil generated by $S_{1}$ and $S_{2}$, the tangency point of $S^{3}$ and $\mathbb{R}^{3}$,which we chose as origin of $\mathbb{R}^{3}$, is the other limit point). Finally, compose the stereographic projection with an homothety to transform the inner sphere into the unit sphere centered at the origin.

Lemma 7.1.12 The knot $K$ is contained in a thin tubular neighborhood of a geodesic circle of the middle sphere $S_{m}$ (defined by the intersection of the ray containing $\frac{1}{2}\left(\sigma_{1}+\sigma_{2}\right)$ and $\Lambda$, where $\sigma_{1}$ and $\sigma_{2}$ are the points in $\Lambda$ corresponding to the spheres $S_{1}$ and $S_{2}$ ) of the zone $Z$.

Proof: We know that the knot $K$ is tangent to $S_{1}$ in two antipodal points, they will be $x_{1}$ and $x_{3}$. We chose $x_{2}$ on the intersection of the knot with the "equatorial" plane associated to the north and south poles $x_{1}$ and $x_{3}$. This defines a strand $\gamma_{1}$, of extremities $x_{1}$ and $x_{3}$, containing $x_{2}$, of $K$. The strand of $K$ joining $x_{3}$ to $x_{1}$ which does not contain $x_{2}$, that we will call $\gamma_{2}$, have to stay in a neighborhood of the arc of extremities $x_{1}$ and $x_{3}$, of the circle defined by the three points $x_{1}, x_{2}, x_{3}$ which does not contain $x_{2}$. Let $x_{4}$ be a point of the intersection of $\gamma_{2}$ with the equatorial plane previously defined. Using now the circle through $x_{1}, x_{2}, x_{4}$ we 
deduce that the strand $\gamma_{1}$ is contained in a neighborhood of the arc of extremities $x_{1}$ and $x_{2}$ of the circle determined by $x_{1}, x_{2}, x_{4}$ which does not contain $x_{4}$.

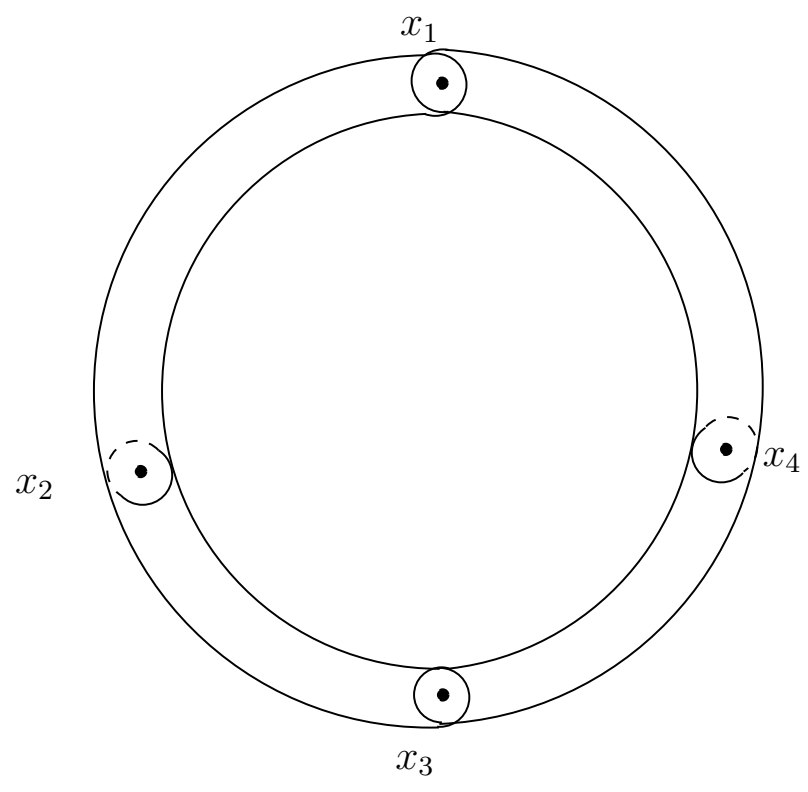

Figure 20: The knot is trapped in a neighborhood of a circle

As the two circles containing $x_{1}, x_{2}, x_{3}$ and containing $x_{1}, x_{2}, x_{4}$ are close to each other, and close to a great circle $\Gamma_{1}$ of the sphere $S_{m}$, which completes the proof.

Consider now the tube of radius $\delta_{1}$ around $\Gamma_{1}$ containing $K$, and geodesic discs $D_{1}^{1}, \ldots D_{n_{1}}^{1}$ of this tube, normal to $\Gamma_{1}$ through equidistant point of $\Gamma_{1}$, say, such that the length of those arc is roughly 100 times the diameter $\delta_{1}$ of the tube. Chose a point $x_{i}^{2}$ of $K$ in each disc $D_{i}^{1}$. Joining consecutive (for the cyclic order) points $x_{i}^{2}$ and $x_{i+1}^{2}$ by a small arc of the circle defined by the two consecutive points $x_{i}^{2}$ and $x_{i+1}^{2}$ and one of the almost antipodal points to $x_{i}^{2}: x_{I}^{2}$, that we will call $\gamma_{i}^{2}$, we get an unknotted polygon $\Gamma_{2}$ with all the angles almost flat (the tangent of those angle is at most, say, $\left.\varphi_{2}<1.5 \cdot \operatorname{Arctg}\left(\frac{1}{100}\right)<\frac{3}{100}\right)$.

Using the the same idea as for the proof of the lemma 7.1.12, we deduce that the "small" arc of the knot joining $x_{i}^{2}$ to $x_{i+1}^{2}$ has to stay in a neighborhood of the small arc of the circle $\gamma_{i}^{2}$ of "diameter" of the order of $\delta_{1} \cdot\left(\right.$ length of $\gamma_{i}^{2}$ ).

The knot is now confined in a thin neighborhood of $\Gamma_{2}$. Let us call $\delta_{2}$ the diameter of this tube, which is also of the order of $\delta_{1} \cdot\left(\right.$ length of $\left.\gamma_{i}^{2}\right)$. Consider now in the tube a sequence of consecutive normal geodesic discs $D_{1}^{2} \ldots D_{n_{2}}^{2}$ of radius $2 \cdot \delta_{2}$. We chose them to be distant distant of roughly $100 \cdot \delta_{2}$. We can define them with no ambiguity, except in a neighborhood of the vertices of $\Gamma_{2}$. The choice of the discs near the vertices of $\Gamma_{2}$ has to be made with more care: they should be roughly at a distance of $50 \delta_{2}$ form the vertices of $\Gamma_{2}$. Again we chose a point $x_{i}^{3}$ in each normal disc $D_{i}^{2}$. We get that way a new unknotted polygon $\Gamma_{3}$. The knot $K$ is now contained in a thin tubular neighborhood of $\Gamma_{3}$. The diameters $\delta_{i}$ of the successive tubes are related to the of one order of magnitude thinner. 


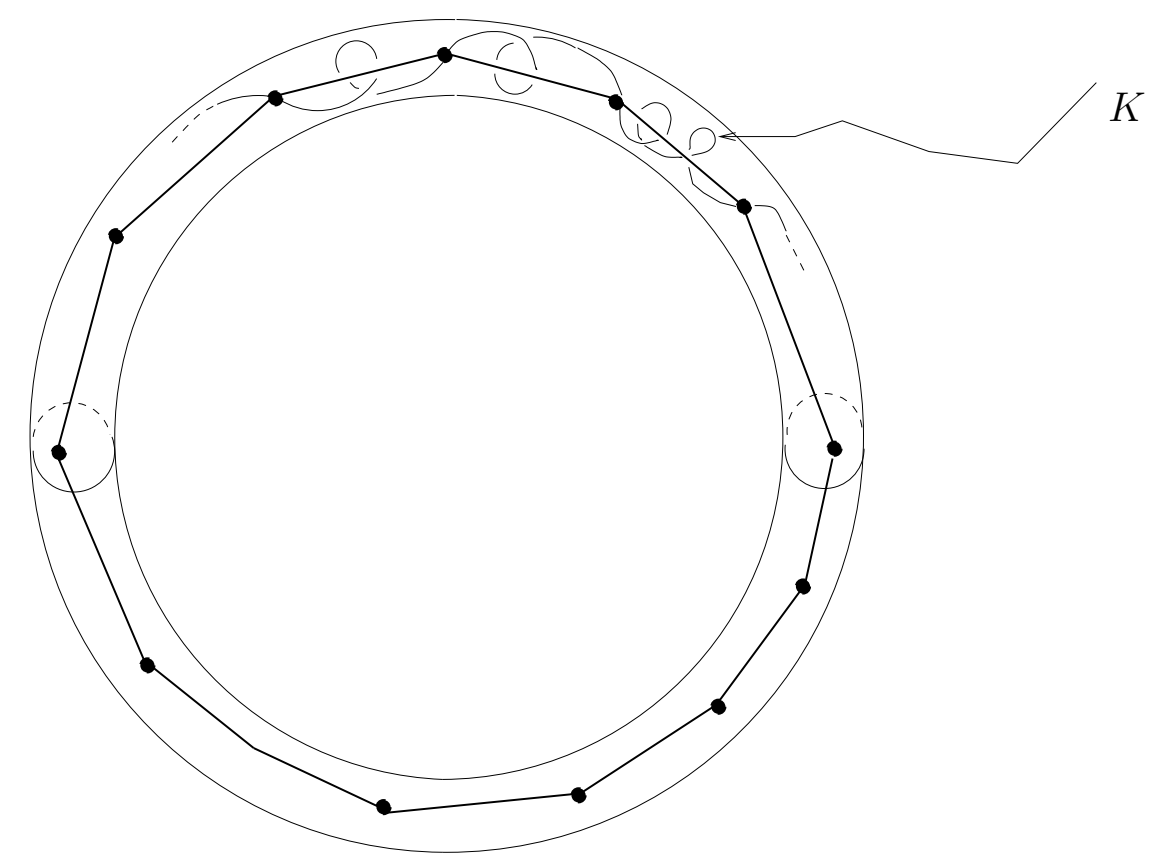

Figure 21: The first polygonal approximation

The only obstruction left to proceed with inductive construction of unknotted polygons inscribed in $K$ is the angles of the polygons. We need to guarantee they stay flat enough. The angles $\theta_{i}^{2}$ of $\Gamma_{2}$ are bounded by $\varphi_{2}$ see next picture.

That is also true for the angles at all the vertices of the successive polygons except for the ones which are adjacent to some vertex of the previous polygon. A priori the angle cannot grow more than linearly with the index of the polygone. In fact $8 \cdot \varphi_{2}$ bounds all the angles of all the $\Gamma_{k}$. If the angle $\theta$ at a vertex of $\Gamma_{k-1}$ is larger than $5 \cdot \varphi_{2}$, then our construction will replace it by two angles of $\Gamma_{k}$ smaller than $\frac{\theta}{2}+3 \varphi_{2}$, therefore never reaching $8 \cdot \varphi_{2}$

Getting to a polygon $\Gamma_{k}$ such that the diameter $\delta_{k}$ of its tubular neighborhood containing the knot $K$ is smaller than, $\frac{1}{3} \cdot \Delta(K)$ where $\Delta(K)$ is the global radius of curvature defined by Gonzalez and Maddocks Go-Ma (see section [8.3), we prove that the knot $K$ is trivial. This ends the proof of the theorem 7.1.11.

Remark: Freedman and He [Fr-He] give a definition of the modulus of a solid torus using the degree of maps from the solid torus $\mathbf{T}$ to the circle $\mathbb{R} / \mathbb{Z}$. From our viewpoint (topology implies a jump of some geometrical invariant), it has the defect of beeing very small when the solid torus is very long and very thin.

But, in the same article Freedman and He defines a dual notion which is bounded below by a positive power of the average crossing number of knot type of $\mathbf{T}$. It would be very interesting to understand possible relations between this modulus they denote by $m^{*}(\mathbf{T})$ and our modulus of a knot, for example via a "thickest" tubular neighbourhood of a representative of a knot in its isotopy class. 


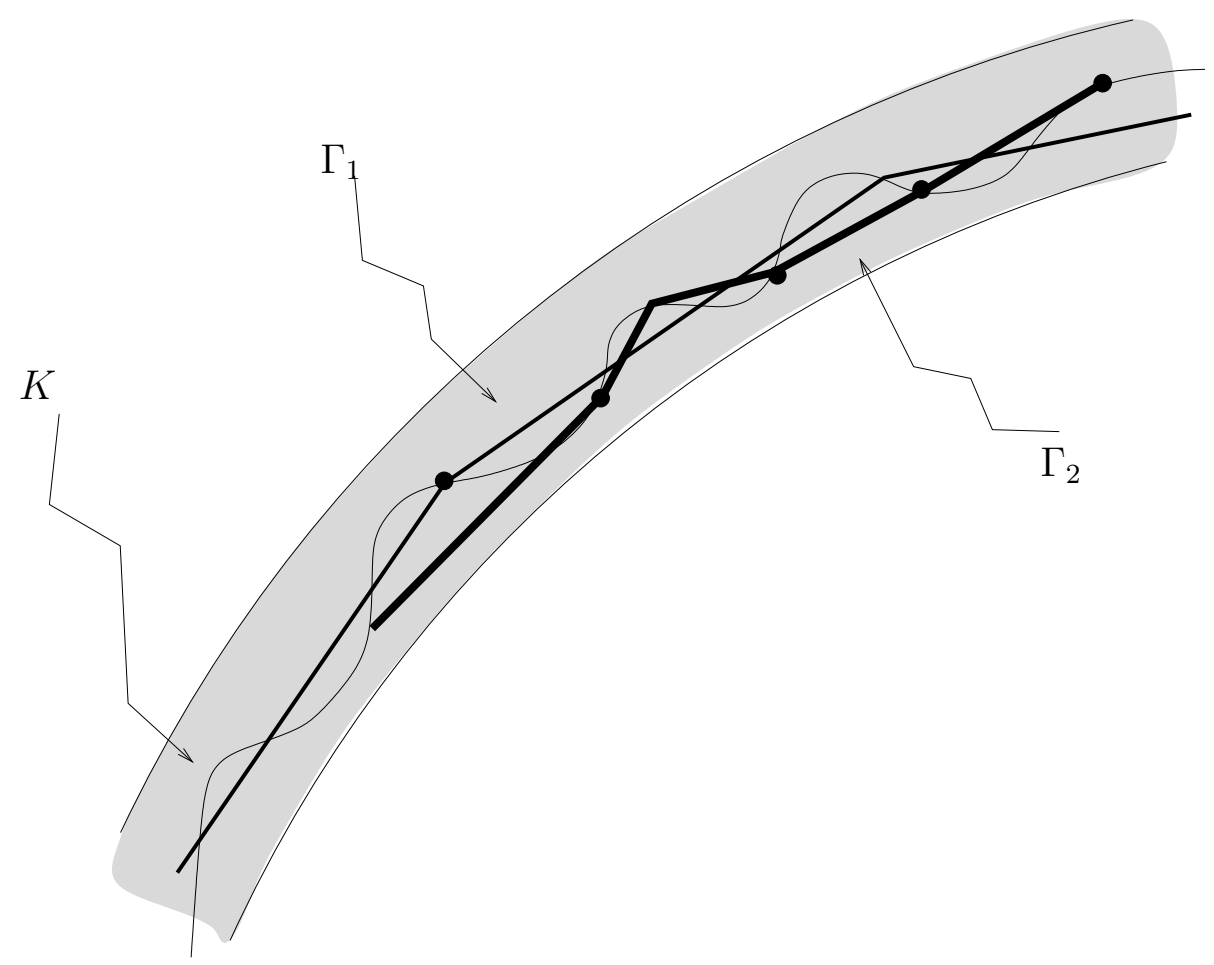

Figure 22: The second polygon $\Gamma_{2}$ inscribed in $K$

\subsection{Jump of the measure of acyclicity for non-trivial knots.}

Given the curve $K$, the spheres contained in a non-trivial zone $A$ intersect $K$ in at least four points. The region $R_{A}$ in $\Lambda$ corresponding to the spheres contained in the annulus $A$ is bounded by two light cones (half light cones to be precise) with vertex the boundary spheres $S_{1}$ and $S_{2}$ of $A$. The volume of $R_{A}$ is a monotonous function of the modulus of $A$. Therefore the existence of a strictly larger than 0 lower bound for the moduli of maximal non-trivial zones associated to non-trivial knots implies the existence of a strictly positive constant $c>0$ such that for any non-trivial knot, the measure $m n t s_{1}(C)$ of the set of non-trivial spheres is larger or equal to $c$.

The modulus and the measure of acyclicity probably do not behave the same way for connected sums of knots. Let $\rho([K])$ (or mnts $([K])$ ) denote the infimum of the modulus (or the measure of acyclicity respectively) of a knot that belongs to an isotopy class $[K]$.

Question 7.2.1 Is it true that:

$$
\rho\left(\left[K_{1} \sharp K_{2}\right]\right)=\max \left\{\rho\left(\left[K_{1}\right]\right), \rho\left(\left[K_{2}\right]\right)\right\} ?
$$

Question 7.2.2 Is it true that:

$$
\operatorname{mnts}\left(\left[K_{1} \sharp K_{2}\right]\right)=(\text { or } \leq \text { or } \geq) \operatorname{mnts}\left(\left[K_{1}\right]\right)+\operatorname{mnts}\left(\left[K_{2}\right]\right) ?
$$

Question 7.2.3 Do the modulus and the measure of acyclicity take their minimum values for non-trivial knots at a (2,3)-torus knot on a torus of revolution? 


\subsection{Explosion of the measure of acyclicity for singular knots.}

Theorem 7.3.1 The measure of acyclicity is an energy functional for knots.

Proof: Fix $\delta\left(0<\delta \leq \frac{1}{2}\right)$. For $0<d \leq \delta$ put

$$
\begin{aligned}
& \mathcal{K}(d)=\mathcal{K}_{\delta}(d)=\left\{\begin{array}{l|l}
K: \text { a knot with } & \begin{array}{l}
\exists x, y \in K \text { such that } \\
\text { length } l(K)
\end{array} \\
& \begin{array}{l}
\text { i) shorter arc-length } \\
\text { between } x \text { and } y \text { is } \delta l(K) . \\
\text { ii) }|x-y| \leq d l(K) .
\end{array}
\end{array}\right\}, \\
& \rho(d)=\inf _{K \in \mathcal{K}(d)}\{\operatorname{mnts}(K)\}
\end{aligned}
$$

We remark that $\rho$ is a monotonely decreasing function of $d$ because $d_{1}<d_{2}$ implies $\mathcal{K}\left(d_{1}\right) \subset$ $\mathcal{K}\left(d_{2}\right)$, and that $\rho(d)=0$ for $d \geq(\sin \pi \delta) / \pi$ because $\mathcal{K}(d)$ contains the standard circle.

Assume that there is a positive constant $M^{\prime}$ such that

$$
\sup _{d>0} \rho(d)=\lim _{d \rightarrow+0} \rho(d)=M^{\prime}
$$

There is a positive constant $M=M\left(M^{\prime}\right)$ such that if the measure of acyclicity of $K$ is smaller than $1.1 M^{\prime}$ then the ratio of the radii of the two spheres of any concentric non-trivial zone for $K$ is smaller than $M$.

Let $K_{d}$ be a knot with length 1 in $\mathcal{K}(d)$ whose measure of acyclicity is smaller than $1.1 M^{\prime}$. Let $x, y$ be a pair of points on $K_{d}$ such that the shorter arc-length between them is $\delta$ and that $d_{0}=|x-y| \leq d .{ }^{10}$ The knot $K$ is divided into two arcs, $A_{1}$ and $A_{2}$, by $x$ and $y$. Put

$$
a=\min \left\{\sup _{z \in A_{1}}\left|z-\frac{x+y}{2}\right|, \sup _{w \in A_{2}}\left|w-\frac{x+y}{2}\right|\right\} .
$$

Let $\Sigma_{r}$ denote the 2 -sphere with center $(x+y) / 2$ and radius $r$. Since $\left(\Sigma_{a}, \Sigma_{d_{0} / 2}\right)$ forms a nontrivial zone, $a \leq M d_{0} / 2$ and hence $a \leq M d / 2$. Let $\bar{K}_{d}$ be the part of $K_{d}$ which is contained in $\Sigma_{M d / 2}$. The length $l\left(\bar{K}_{d}\right)$ of $\bar{K}_{d}$ is greater than or equal to $\delta$ since $\Sigma_{M d / 2}$ contains at least one of the $\operatorname{arcs} A_{1}$ and $A_{2}$. Then by Poincaré's formula ([Sa] page 259, see also pages 111, 277) there holds

$$
\int_{G} \#\left(g\left(\Sigma_{r}\right) \cap \bar{K}_{d}\right) d g=\frac{O_{3} O_{2} O_{1} \cdot O_{0}}{O_{2} O_{1}} \cdot \operatorname{area}\left(\Sigma_{r}\right) \cdot l\left(\bar{K}_{d}\right),
$$

where $O_{n}$ denotes the volume of the $n$ dimensional unit sphere, $G$ denotes the group of the orientation preserving motions of $\mathbb{R}^{3}$, which is isomorphic to the semidirect product of $\mathbb{R}^{3}$ and $S O(3)$, and $d g$ denotes the kinematic density ([Sa] page 256). We have $d g=d P \wedge d K_{[P]}$, where $P \in \mathbb{R}^{3}, d P$ is the volume element of $\mathbb{R}^{3}$, and $d K_{[P]}$ is the kinematic density of the group of special rotations around $P$, which is isomorphic to $S O(3)$. Since

$$
\int d K_{[P]}=O_{2} O_{1}
$$

\footnotetext{
${ }^{10}$ We use the axiom of choice here.
} 
the Poincaré's formula implies

$$
\int_{\Sigma_{r}(X, Y, Z) \cap K_{d} \neq \phi} \#\left(\Sigma_{r}(X, Y, Z) \cap K_{d}\right) d X d Y d Z=2 \pi r^{2} l\left(\bar{K}_{d}\right),
$$

where $\Sigma_{r}(X, Y, Z)$ denotes the sphere with radius $r$ and center $(X, Y, Z)$. Suppose $d / 2 \leq r \leq$ $M d / 2$. Then the measure of acyclicity of $K_{d}$ satisfies

$$
\begin{aligned}
\operatorname{mnts}\left(K_{d}\right) & =\int_{N T\left(K_{d}\right)} C_{2}^{\#\left(\Sigma_{r}(X, Y, Z) \cap K_{d}\right)} \cdot \frac{1}{r^{4}} d X d Y d Z d r \\
& \geq \int_{d / 2}^{M d / 2} \frac{1}{r^{4}}\left\{\int_{D_{M d / 2+r}^{3}((x+y) / 2)} \frac{\#\left(\Sigma_{r}(X, Y, Z) \cap \bar{K}_{d}\right)-1}{2} d X d Y d Z\right\} d r,
\end{aligned}
$$

where $D_{c}^{3}(R)$ denotes the 3 -ball with center $c$ and radius $R$. Thus

$$
\begin{aligned}
\operatorname{mnts}\left(K_{d}\right) & \geq \frac{1}{2} \int_{d / 2}^{M d / 2} \frac{1}{r^{4}}\left\{2 \pi r^{2} l\left(\bar{K}_{d}\right)-\frac{4}{3} \pi\left(\frac{M d}{2}+r\right)^{3}\right\} d r \\
& \geq \frac{1}{2}\left\{2 \pi \delta\left[-\frac{1}{r}\right]_{d / 2}^{M d / 2}-\frac{4}{3} \pi(M d)^{3}\left[-\frac{1}{3 r^{3}}\right]_{d / 2}^{M d / 2}\right\}
\end{aligned}
$$

which blows up as $d$ goes down to 0 , which contradicts the assumption that $\lim _{d \rightarrow+0} \rho(d) \leq M<\infty$.

\section{Appendix.}

\subsection{The maximal modulus and cross-ratio of four points.}

In this subsection we give a formula to express the maximal Lorentzian modulus $\mu_{L}\left(x^{1}, x^{2}, x^{3}, x^{4}\right)$ of the four points in terms of the cross-ratio $\left(x^{2}, x^{3} ; x^{1}, x^{4}\right)$. It implies that the integrand of $E^{(2)}$ is equal to the infinitesimal maximal modulus $\mu_{L}(x, x+d x, y, y+d y)$.

But we start with an alternative proof of Lemma [7.1.9] by showing it in lower dimension by one. The terms in the following lemma can be defined in a parallel way.

Lemma 8.1.1 Let $x_{1}, x_{2}, x_{3}$, and $x_{4}$ be ordered four points on $S^{2}$. Suppose the cross-ratio $\left(x_{2}, x_{3} ; x_{1}, x_{4}\right)$ is not a real number between 0 and 1 .

Then

(1) The maximal modulus of a separating annulus is attained by a separating annulus, which will be called a maximal separating annulus. Then the pair $x_{1}, x_{3}$ (or $\left.x_{2}, x_{4}\right)$ is on a circle of the pencil whose base points are the limit points of the pencil defined by the boundary circles of the maximal separating annulus.

(2) There is exactly one maximal separating annulus. The position of the boundary circles of the annulus is explicitely determined in fonction of the four points. 
Proof: (1) First observe that the two boundary circles $C_{1}$ and $C_{2}$ of a separating annulus with the maximal modulus should contain, one $x_{1}$ and $x_{3}$, the other $x_{2}$ and $x_{4}$. The idea of the proof is the same as the idea of the proof of Lemma 7.1.9. If these points where not on a circle $\Gamma$ through the limit point generated by the two boundary circles, say for $x_{1} \in C_{1}$ and $x_{3} \in C_{1}$, rotating from $C_{1}$ in the pencil of base points $x_{1}$ and $x_{3}$ will define a curve in the quadric $\Lambda$ of circles in $S^{2}$ transverse at $C_{1}$ to the level $L\left(C_{2}, C\right)=L\left(C_{2}, C_{1}\right)$ of $L$. Therefore this curve is transverse to the level $m\left(C_{2}, C\right)=m\left(C_{2}, C_{1}\right)$, contradicting the fact that a maximum is a critical point.

(2) Let $\mathbb{P} \Lambda$ be the set of non-oriented spheres $\mathbb{P} \Lambda=\Lambda / \pm 1$. The pencils of circles through $x_{1}, x_{3}$ and through $x_{2}, x_{4}$ can be seen as two circles $\gamma_{13}$ and $\gamma_{24}$ in $\mathbb{P} \Lambda$. Consider the four circles determined by three of the four points $x_{1}, x_{2}, x_{3}, x_{4}$. Two of them bound on $\gamma_{13}$ an open segment: the set of circles contained in the pencil $\gamma_{13}$ separating (in $S^{2}$ ) $x_{1}, x_{3}$ from $x_{2}, x_{4}$. We have a similar situation on the pencil $\gamma_{24}$.

Let us prove that the boundary circles of a maximal annulus are the middle points (for the arc-length in $\mathbb{P} \Lambda$ ) of the segments we just defined. This proves the uniqueness of the maximal separating annulus.

The arc-length on a pencil in $\mathbb{P} \Lambda$ is is just the angle through a base point of the pencil.

On the picture 8.1 we have represented the pencil $\gamma_{24}$, the circles $C_{124}$ through $x_{1}, x_{2}, x_{4}$ and $C_{324}$ through $x_{3}, x_{2}, x_{4}$, and the circles $S_{1}$ and $S_{2}$, boundary of a maximal separating annulus.

The angles between $C_{124}$ and $S_{2}$ and the angle between $C_{324}$ and $S_{2}$ are equal, since the picture is symmetric with respect to the origin and since angles on two sides of a point of intersection of two circles are equal. That completes the proof.

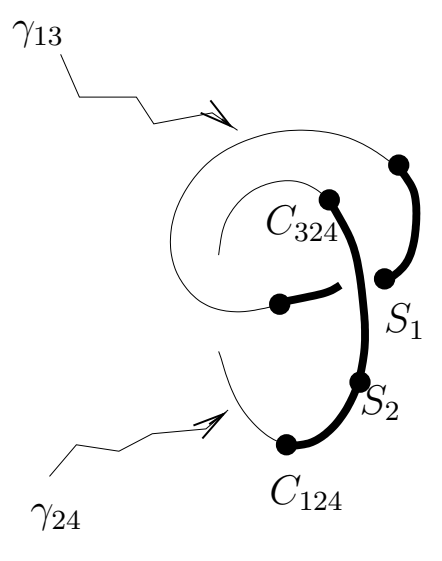

$($ in $\mathbb{P} \Lambda)$

(a)

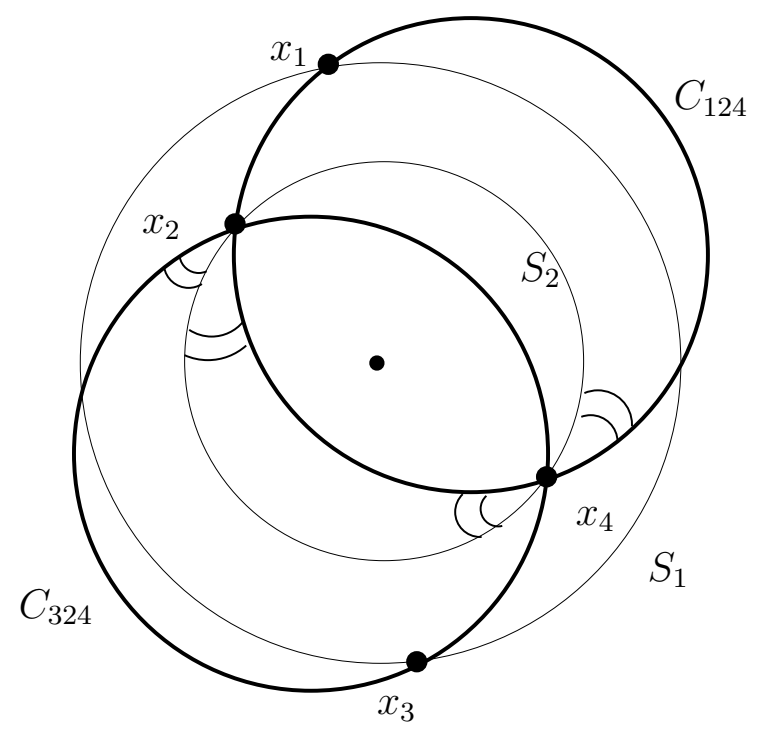

(b)

Figure 23: Characterization of the boundary circles of a maximal separating annulus 
Lemma 8.1.2 Let $C_{1}$ and $C_{2}$ be a pair of concentric circles on $\mathbb{R}^{2} \subset \mathbb{R}^{3}$. Then among the zones $Z$ with $\partial Z=S_{1} \cup S_{2}$ satisfying $\left(S_{1} \cup S_{2}\right) \cap \mathbb{R}^{2}=C_{1} \cup C_{2}$, the maximal modulus is attained by the unique zone which has concentric boundary spheres whose center is in the plane $\mathbb{R}^{2}$; these spheres are therefore orthogonal to the plane $\mathbb{R}^{2}$.

Proof: Just notice that, when we presented (in lemma [7.1.9) a maximal non-trivial zone using two concentric spheres, the four pairwise antipodal points $x_{1}, x_{3}$ and $x_{2}, x_{4}$ belong the the same plane, which is othogonal to the two concentric spheres.

Corollary 8.1.3 Let $x_{1}, x_{2}, x_{3}$, and $x_{4}$ be ordered four points in $\mathbb{R}^{3}$ or $S^{3}$, and let $\Sigma$ be a 2sphere passing through them. Then the maximal modulus $\mu\left(x_{1}, x_{2}, x_{3}, x_{4}\right)$ of the four points is equal to the maximal modulus of a separating annulus in $\Sigma$.

Claim 8.1.4 The maximal Lorentzian modulus $\mu_{L}\left(x^{1}, x^{2}, x^{3}, x^{4}\right)$ is expressed in terms of the cross-ratio $\mathrm{cr}=\left(x^{2}, x^{3} ; x^{1}, x^{4}\right)$ as

$$
\mu_{L}\left(x^{1}, x^{2}, x^{3}, x^{4}\right)=\sqrt{1+2|1-c r|^{2}\left\{\left|\frac{c r}{1-c r}\right|-\Re\left(\frac{c r}{1-c r}\right)\right\}} .
$$

Remark: Lemma 7.1.9 implies the above Claim as follows. The boundary spheres of the maximal separating zone can be mapped into a concentric position by a conformal transformation. Then Lemma [7.1.9 asserts that $x_{1}$ and $x_{3}$ are antipodal in one of the boundary spheres, and $x_{2}$ and $x_{4}$ are antipodal in another. We can assume without loss of generality that $x_{1}, x_{3}= \pm 1$ and $x_{2}, x_{4}= \pm z$ in $\mathbb{C} \subset \mathbb{R}^{3}$, and the boundary spheres of the maximal separating zone are concentric spheres with center the origin and radii 1 and $|z|$. Then the maximal Lorentzian modulus $\mu_{L}\left(x^{1}, x^{2}, x^{3}, x^{4}\right)$ is given by $(|z|+1 /|z|) / 2$ and the cross-ratio $c r=\left(x^{2}, x^{3} ; x^{1}, x^{4}\right)$ is given by $-(z-1)^{2} /(4 z)$, and therefore $c r /(1-c r)=(z-1)^{2} /(z+1)^{2}$. One can verify the equality by the direct calculation.

Lemma 8.1.5 Let $\Pi$ be a plane and $\Sigma$ be a 2-sphere in $\mathbb{R}^{3}$ such that the minimum and maximum distance between $\Pi$ and $\Sigma$ are $a$ and $b(0<a<b)$ respectively. Then the modulus $\rho(\Sigma, \Pi)$ of the zone bounded by $\Sigma$ and $\Pi$ is given by

$$
\rho(\Sigma, \Pi)=\log \left(\frac{\sqrt{b}+\sqrt{a}}{\sqrt{b}-\sqrt{a}}\right) .
$$

Proof: May assume that $\Pi$ is the $y$ - $z$ plane and $\Sigma$ has $\left(\frac{a+b}{2}, 0,0\right)$ as its center. Then any inversion with respect to a 2 -sphere with center $( \pm \sqrt{a b}, 0,0)$ maps $\Sigma$ and $\Pi$ to a concentric position.

This Lemma immediately implies the following Lemma.

Lemma 8.1.6 Let $\Sigma$ be a 2-sphere in $\mathbb{R}^{3}$ with center $O$, and $A \in \mathbb{R}^{3}$ be a point outside $\Sigma$. Then among planes $P$ passing through $A$, the plane $P_{0}$ perpendicular to the line $O A$ gives the maximal modulus $\rho(\Sigma, P)$. 
Let $T_{0}$ be an inversion with respect to a 2 -sphere whose center lies on the line $O A$ that maps $\Sigma$ and $P_{0}$ to a concentric position as is given in the proof of the previous Lemma. Then $T_{0}(A)$ and $T_{0}(\infty)$ are antipodal in $T_{0}\left(P_{0}\right)$. Therefore this lemma gives an alternative computational proof of Lemma [7.1.9.

Lemma 8.1.7 Let $C$ be a circle with center $O$ in a plane $\Pi$, and $A \in \Pi$ be a point outside $C$. Then among pairs of a sphere $\Sigma$ passing through $C$ and a plane $P$ passing through $A$, the pair of the sphere $\Sigma_{0}$ whose center belongs to $\Pi$ and the plane $P_{0}$ perpendicular to the line $O A$ gives the maximal modulus.

Proof: Let $r$ be the radius of $C$ and $a=|O-A|$. Let $\Sigma(h)$ denote the 2-sphere whose center is apart from $\Pi$ by $h(h \geq 0)$. Then the preceding two Lemmas imply that the maximum of the modulus $\rho(\Sigma(h), P)$ with $P \ni A$ is given by

$$
\log \left(\sqrt{1+\frac{a^{2}-r^{2}}{r^{2}+h^{2}}}+\sqrt{\frac{a^{2}-r^{2}}{r^{2}+h^{2}}}\right)
$$

which is a decreasing function of $h$.

Proof of Claim 8.1.4 Without loss of generality we can assume that $x^{1}=\infty, x^{2}=0$, $x^{3}=1$, and $x^{4}=z=u+i v$ in $\mathbb{C} \cup\{\infty\} \cong \mathbb{R}^{2} \cup\{\infty\} \subset \mathbb{R}^{3} \cup\{\infty\}$. Then the cross-ratio $c r=(0,1 ; \infty, z)=1-\frac{1}{z}$ and hence $\frac{c r}{1-c r}=z-1$. We assume that $z \notin\{z \in \mathbb{R} \mid z>1\}$, otherwise the both sides of the formula coincide with 1 . The previous lemma implies that the maximal modulus $\mu_{L}(\infty, 0,1, z)$ is attained by a zone $Z(\xi)$ bounded by $S_{1}(\xi)$ and $S_{2}(\xi)$ for some $\xi \in \mathbb{R}$, where $S_{2}(\xi)$ has center

$$
O(\xi)=\left(\frac{u}{2}-\frac{v}{\sqrt{u^{2}+v^{2}}} \xi, \frac{v}{2}+\frac{u}{\sqrt{u^{2}+v^{2}}} \xi\right) \in \mathbb{R}^{2},
$$

and $S_{1}(\xi)$ is a plane perpendicular to the line joining $O(\xi)$ and 1 . Then $\rho\left(S_{1}(\xi), S_{2}(\xi)\right)$ is given by

$$
\rho(Z(\xi))=\log \left(\frac{\sqrt{1-u+\frac{u^{2}+v^{2}}{4}+\frac{2 v}{\sqrt{u^{2}+v^{2}}} \xi+\xi^{2}}+\sqrt{1-u+\frac{2 v}{\sqrt{u^{2}+v^{2}}}} \xi}{\sqrt{\frac{u^{2}+v^{2}}{4}+\xi^{2}}}\right),
$$

which takes the maximum value

$$
\frac{1}{2} \log \left(1+\frac{2\left\{\sqrt{(u-1)^{2}+v^{2}}-(u-1)\right\}}{u^{2}+v^{2}}\right) .
$$

As a corollary of Claim 8.1.4 there holds:

Corollary 8.1.8 The integrand $\left|\Omega_{C R}\right|-\Re_{C R}$ of $E^{(2)}(K)$ can be interpreted as the maximal Lorentzian modulus $\mu_{L}(x, x+d x, y, y+d y)-1$ of a pair of infinitesimal segments. 


\subsection{The circular Gauss map and the inverted open knots.}

Let $C(y, y, x)$ be as in definition 3.3.1 and $v_{y}(x)$ be as in subsection 5.1. We remark that when $y$ approaches $x$ then $C(y, y, x)$ approaches the osculating circle at $x$, which will be denoted by $C(x, x, x)$, and $v_{y}(x)$ approaches the tangent vector at $x$, which will be denoted by $v_{x}(x)$.

Definition 8.2.1 The circular Gauss map $\stackrel{\circ}{\Phi}_{K}$ of a knot $K$ is defined by

$$
\stackrel{\circ}{\Phi}_{K}: S^{1} \times S^{1} \ni(s, t) \mapsto v_{f(s)}(f(t)) \in S^{2}
$$

We remark that $\stackrel{\circ}{\Phi}_{K}$ is well-defined at the diagonal set, but it is not a symmetric function. Remark: The circular Gauss map $\stackrel{\circ}{\Phi}_{K}$ is not conformally invariant, namely, for a conformal transformation $T$ there is generally no element $g$ in $O(3)$ that satisfies $\stackrel{\circ}{\Phi}_{T(K)}(s, t)=g\left(\stackrel{\circ}{\Phi}_{K}\right.$ $(s, t))$ for any $(s, t) \in S^{1} \times S^{1}$. To see this one can put $T=I_{x}$ as an extreme case, when " $v_{T(x)}(T(y)) "=I_{x *}\left(v_{x}(y)\right)$ is constantly equal to $-v_{x}(x)$.

Claim 8.2.2 The degree of $\stackrel{\circ}{\Phi}_{K}$ vanishes for any knot $K$.

Proof: Any knot $K$ can be deformed continuously by an ambient isotopy to a very "thin position" so that $K$ is contained in $x y$-plane except for some 'bridges' which are contained in $\{0 \leq z \leq \epsilon\}$ as illustrated in Figure 24. Then the preimage of the vector $(0,0,1) \in S^{2}$ by $\stackrel{\circ}{\Phi}_{K}$ consists of pairs of points near the crossing points.

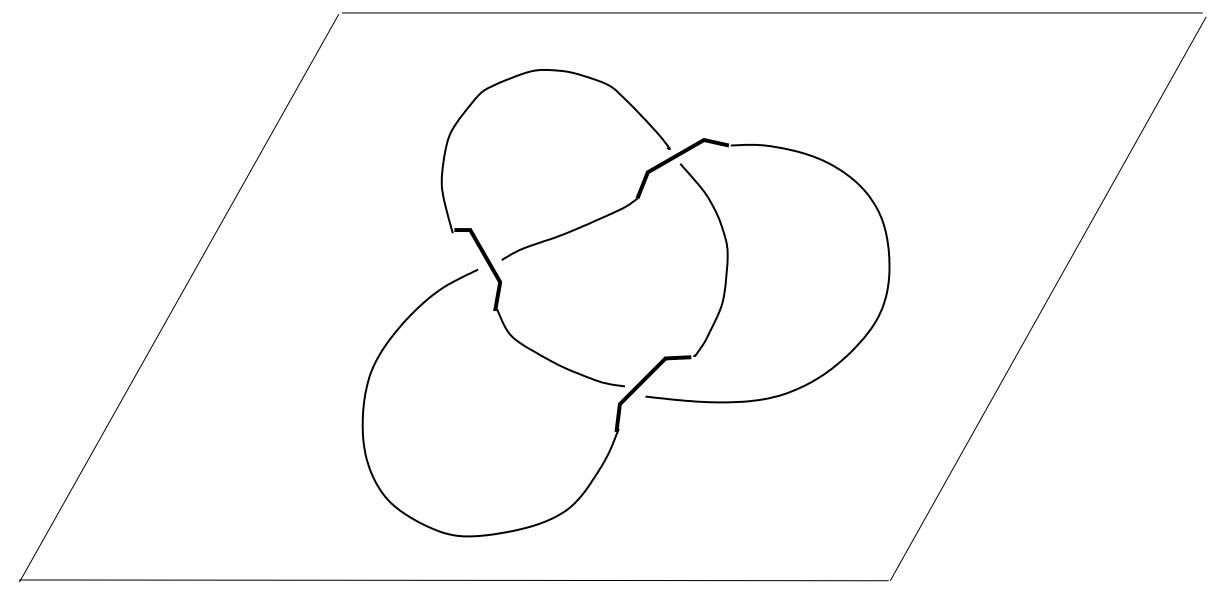

Figure 24: A knot in "thin position".

Suppose a knot satisfies

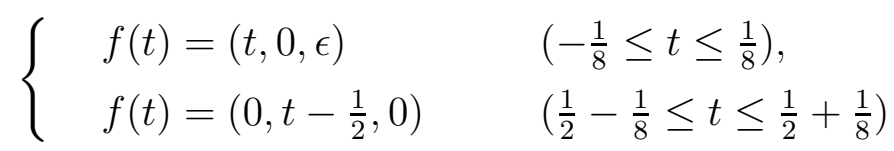


for a small $\epsilon\left(0<\epsilon<\frac{1}{8}\right)$. Put

$$
U=\left[-\frac{1}{8}, \frac{1}{8}\right] \cup\left[\frac{1}{2}-\frac{1}{8}, \frac{1}{2}+\frac{1}{8}\right]
$$

The preimage $\left(\left.\stackrel{\circ}{\Phi}_{K}\right|_{U \times U}\right)^{-1}((0,0,1)) \in U \times U$ is a pair of points $\left(\epsilon, \frac{1}{2}\right)$ and $\left(\frac{1}{2}-\epsilon, 0\right)$, where the signatures of $\left(\left.\stackrel{\circ}{\Phi}_{K}\right|_{U \times U}\right)_{*}$ are opposite, hence the contribution of $U \times U$ to the degree of $\stackrel{\circ}{\Phi}_{K}$ is 0 .
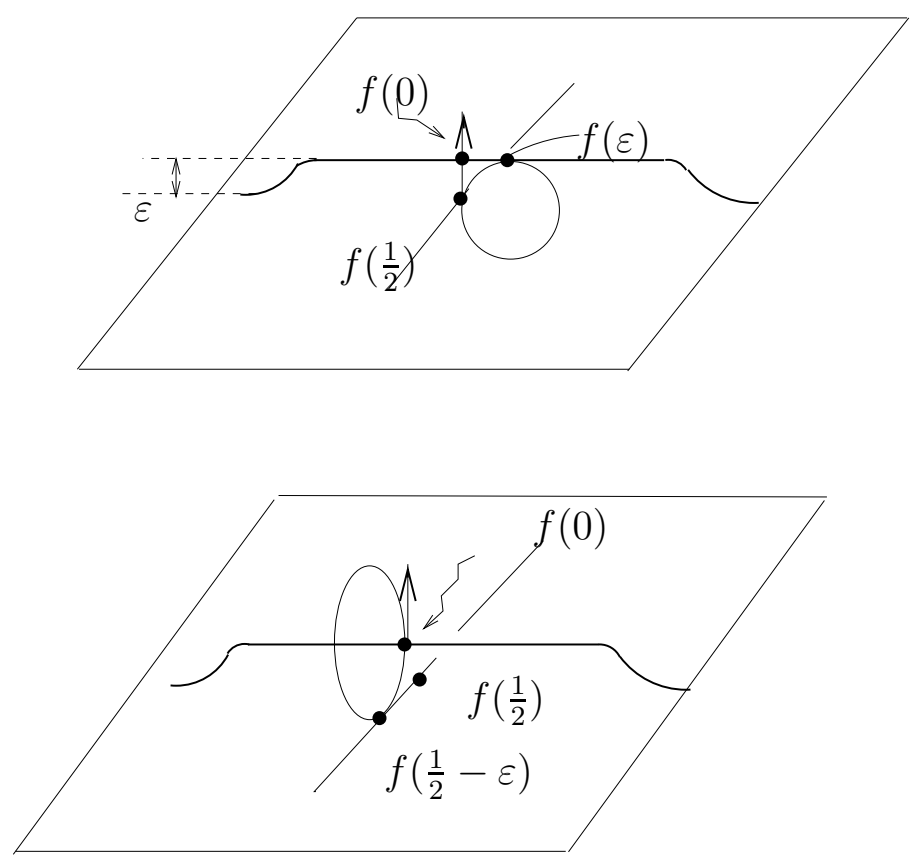

Figure 25: $\left(\stackrel{\circ}{\Phi}_{K}\right)^{-1}((0,0,1))$.

Let us introduce two non-trivial functionals derived from $\stackrel{\circ}{\Phi}_{K}$.

Definition 8.2.3 (1) Let $\omega_{S^{2}}$ be the unit volume 2-form of $S^{2}$ :

$$
\omega_{S^{2}}=\frac{1}{4 \pi} \frac{x_{1} d x_{2} \wedge d x_{3}+x_{2} d x_{3} \wedge d x_{1}+x_{3} d x_{1} \wedge d x_{2}}{\left(x_{1}^{2}+x_{2}^{2}+x_{3}^{2}\right)^{\frac{3}{2}}} .
$$

The absolute circular self-linking number à la Gauss, $\mid$ csl $\mid$, is defined by

$$
|\operatorname{csl}|(K)=\int_{T^{2}}\left|\stackrel{\circ}{\Phi}_{K}^{*}\left(\omega_{S^{2}}\right)\right| .
$$

(2) Let $l_{K}(x)$ be the length of the curve $\cup_{y \in K} v_{y}(x)=\stackrel{\circ}{\Phi}_{K}\left(S^{1}, s\right)$ on $S^{2}$. The total variation of the conformally translated tangent vectors, $I_{t v}$, is defined by

$$
I_{t v}(K)=\int_{S^{1}} l_{K}(x) d x
$$


By definition $I_{t v}(K) \geq|c s l|(K)$ for any knot $K$.

Let us give geometric interpretation of $I_{t v}$ using the inverted open knot. Let $I_{x}, \tilde{K}_{x}$, and $\tilde{y}$ be as in subsection 4. Then $I_{x}(C(y, y, x))$ is the (oriented) tangent line to $\tilde{K}_{x}$ at $\tilde{y}$. Let $\tilde{v}_{\tilde{y}}(\tilde{y})$ be the unit tangent vector to $I_{x}(C(y, y, x))$ at $\tilde{y}$. Then $-v_{y}(x)=I_{x *}\left(v_{y}(x)\right)=\tilde{v}_{\tilde{y}}(\tilde{y})$. Therefore $l_{K}(x)$ is equal to the length of the curve $\cup_{\tilde{y} \in \tilde{K}_{x}} \tilde{v}_{\tilde{y}}(\tilde{y}) \subset S^{2}$. Thus $I_{t v}(K)$ can be considered as the total variation of the tangent vectors to the inverted open knots.

If $T: \mathbb{R}^{3} \cup\{\infty\} \rightarrow \mathbb{R}^{3} \cup\{\infty\}$ is a conformal transformation that fixes $\{\infty\}$, then $\left.T\right|_{\mathbb{R}^{3}}$ is a homothety. Therefore we have:

Claim 8.2.4 The homothety class of the inverted open knot $\tilde{K}_{x}$ is a conformal invariant of $K$. Namely, $I_{T(x)}(T(K))$ is homothetic to $\tilde{K}_{x}$ for any conformal transformation $T$.

Therefore the functional $I_{t v}$ is a conformally invariant functional.

Although $I_{t v}$ is conformally invariant, it can not be expressed in terms of the infinitesimal cross-ratio like $E^{(2)}$ and $E_{\sin \theta}$, because $I_{t v}$ is determined by up to the second order derivatives of the knot.

Claim 8.2.5 Neither $\mid$ csl $\mid$ nor $I_{t v}$ is an energy functional for knots.

Proof: Suffice to show that $I_{t v}$ is not an energy functional because $I_{t v} \geq|c s l|$ for any knot.

We show that the contribution to $I_{t v}$ of a pair of straight line segments of a fixed length with the closest distance $\epsilon$ does not blow up even if $\epsilon$ goes down to 0 . Let $A_{1}=\{x(s) \mid a \leq s \leq b\}$ and $A_{2}=\left\{y(t) \mid a^{\prime} \leq t \leq b^{\prime}\right\}$ be straight line segments with $\min _{x \in A_{1}, y \in A_{2}}|x-y|=\epsilon$. Let us consider $\left.I_{t v}\right|_{A_{1} \cup A_{2}}$. Put

$$
u=f^{\prime}(s), v=f^{\prime}(t), \text { and } w=\frac{f(t)-f(s)}{|f(t)-f(s)|}
$$

as before. Then $u$ and $v$ are constant. Fix $t$ and put $\gamma(s)=v_{x}(y)$. Then as $\gamma(s)=2(u, w) w-u$

$$
\frac{d \gamma}{d s}=2\left(u, \frac{d w}{d s}\right) w+2(u, w) \frac{d w}{d s}
$$

hence

$$
\left|\frac{d \gamma}{d s}\right| \leq 2 \sqrt{2}\left|\frac{d w}{d s}\right|
$$

Therefore

$$
l_{A_{1}}(y)=\int_{a}^{b}\left|\frac{d \gamma}{d s}\right| d s \leq 2 \sqrt{2} \int_{a}^{b}\left|\frac{d w}{d s}\right| d s \leq 2 \sqrt{2} \pi
$$

and hence

$$
\left.I_{t v}\right|_{A_{1} \cup A_{2}}=\int_{a^{\prime}}^{b^{\prime}} l_{A_{1}}(y) d t+\int_{a}^{b} l_{A_{2}}(x) d s \leq 2 \sqrt{2} \pi\left(b^{\prime}-a^{\prime}+b-a\right),
$$

which is independent of $\epsilon$.

We show that they can detect the unknot. 
Example 8.2.6 Let $K_{\circ}$ be the standard planar circle. Then $v_{y}(x)=f^{\prime}(s)$ for any $y$ and therefore $I_{t v}\left(K_{\circ}\right)=|c s l|\left(K_{\circ}\right)=0$

Theorem 8.2.7 If $K$ is a non-trivial knot then $I_{t v}(K) \geq \pi$.

Proof: The unit tangent vector $\tilde{v}_{\tilde{y}}(\tilde{y})$ of the inverted open knot $\tilde{K}_{x}$ is asymptotic to $-v_{x}(x)$ as $\tilde{y}$ goes to $\infty$. If the angle between $\tilde{v}_{\tilde{y}}(\tilde{y})$ and $-v_{x}(x)$ is smaller than $\frac{\pi}{2}$ for any $\tilde{y}$ then $\tilde{K}_{x}$ is unknotted, which contradicts the assumption. Therefore $l_{K}(x) \geq \pi$ for any $x \in K$.

Conjecture 8.2.8 There is a positive constant $C$ such that if $K$ is a non-trivial knot then $|\operatorname{csl}|(K) \geq C$.

\subsection{The global radius of curvature.}

Let us make a remark on the relation between the infimum of the radii of non-trivial spheres and the global radius of curvature by Gonzalez and Maddocks (Go-Ma $)$. Let us consider a knot $K$ in $\mathbb{R}^{3}$.

Let $r(C(x, y, z))$ be the radius of the circle $C(x, y, z)$ that passes through $x, y$ and $z$ in $K$. When two (or three) of them coincide $C(x, y, z)$ means the tangent (or respectively, osculating) circle. The global radius of curvature $\rho_{K}^{(3)}(x)$ of a knot $K$ at $x$ is defined by

$$
\rho_{K}^{(3)}(x)=\min _{y, z \in K} r(C(x, y, z))
$$

This $\rho_{K}^{(3)}(x)$ is attained by a triple $(x, y, y)$, and especially, when it is attained by a triple $(x, x, x), \rho_{K}^{(3)}(x)$ is the radius of curvature in the ordinary sense.

Let $\sigma(x, y, z, w)$ denote the smallest sphere that passes through $x, y, z$ and $w$ in $K$, where the points in $\sigma \cap K$ are counted with multiplicity according to the order of the tangency. Generically $\sigma(x, y, z, w)$ is uniquely determined. Let $r(\sigma(x, y, z, w))$ be the radius of $\sigma(x, y, z, w)$. Put

$$
\rho_{K}^{(4)}(x)=\inf _{(y, z, w) \in K} r(\sigma(x, y, z, w)) .
$$

Since $r(\sigma(x, y, z, w)) \geq r(C(x, y, z))$ there holds $\rho_{K}^{(4)}(x) \geq \rho_{K}^{(3)}(x)$. Put

$$
\begin{aligned}
& \triangle(K)=\min _{x \in K} \rho_{K}^{(3)}(x)=\min _{x, y, z \in K} r(C(x, y, z)), \\
& \square(K)=\inf _{x \in K} \rho_{K}^{(4)}(x)=\inf _{(x, y, z, w) \in K} r(\sigma(x, y, z, w)) .
\end{aligned}
$$

Then $\triangle(K)$ is equal to the thickness studied by Buck and Simon et al. As is shown in [Go-Ma] $\triangle(K)$ is either the minimum local radius of curvature or the strictly smaller radius of the sphere $\sigma=\partial D^{3}$ with $\operatorname{Int} D^{3} \cap K=\phi$ which is twice tangent to $K$ at antipodal points $x$ and $y$.

Claim 8.3.1 Ma-Sm Go-Ma-Sm $\rho_{K}^{(3)}(x)=\rho_{K}^{(4)}(x)$ for any $x$. Therefore $\triangle(K)=\square(K)$. 


\subsection{Links}

One can ask similar questions for links $\mathcal{L}=C_{1} \cup C_{2}$

We say that the link is splittable if there exist two disjoint closed balls $B_{1}$ and $B_{2}$ such that $C_{1} \subset B_{1}$ and $C_{2} \subset B_{2}$.

1. What can be said about the measure of spheres (non-trivial spheres) intersecting the two components if the link is not splittable?

2. Define a non-trivial zone for a link as a zone crossed by the two components (then at least two stands of one and two strands of the other component cross the zone), and the modulus of a link as the maximum of the modulus of a non-trivial zone for the link. Give a lower bound for the modulus of a non-splittable link.

A recent result of Langevin and Moniot [La-Mo] is:

Claim 8.4.1 The modulus of a non-splittable link is bounded below by the modulus of the Hopf Link:

$$
\mathcal{H}=C_{1} \cup C_{2} ; C_{1}=\{x=0\} \cap S^{3}, C_{2}=\{y=0\} \cap S^{3} ; S^{3} \subset \mathbb{C}
$$

As for knots, the claim provides a lower bound for the measure of non-trivial spheres for a non-splittable link. 


\section{References}

[A-K] V. I. Arnold and B. A. Khesin. Topological Methods in Hydrodynamics. Applied Math. Sci. 125, Springer, New York (1998).

[Au-Sa] D. Auckly. and L. Sadun. A family of conformal invariant 2-knot energies. Geometric Topology (Proceedings of the 1993 Georgia International Topology Conference), AMS/IP Studies in Adv. Math., W. H. Kazez ed; Amer. Math. Soc. and International Press, Cambridge, MA, (1997) p.235-258.

[Ban] T. Banchoff. Total central curvature of curves. Duke Math. J. 37 (1970) p.281-289.

[Bar] J. E. Barbier. Note sur le problème de l'aiguille et le jeu du joint ouvert. Journal de mathématiques pures et appliquées (2) 5 (1860) p.534-541.

[Ca-Sh-We] G. Cairns, R. Sharpe, and L. Webb. Conformal invariants for curves and surfaces in three dimensional space forms. Rocky Mountain J. of Math. 24 (1994) p.933-959.

[Ca-Mi-Ra] J. A. Calvo, K. Millett, and E. Rawdon eds., Contemp. Math. 304, Amer. Math. Soc., Providence, RI (2002) p.195-215.

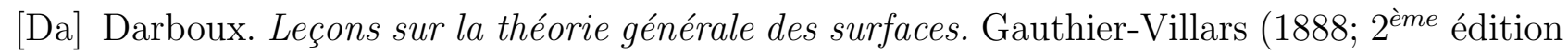
1915).

[Fa] I. Fary. Sur la courbure totale d'une courbe gauche faisant un noeud. Bulletin de la société mathématique de France 77 (1949) p.128-138.

[Fe1] W. Fenchel. Uber Krümmung und Windung geschlössener Raum Kurven. Math. Ann. 101 (1929) p.238-252.

[Fe2] W. Fenchel. On the differential geometry of closed curves. Bull. of Amer. Math. Soc. 15 (1951) p.44-54.

[Fr-He] M.H. Freedman and Z-X. He. Divergence-free fields: Energy and asymptotic crossing number. Ann. of math. 134 (1991) p.189-229.

[Fr-He-Wa] M.H. Freedman, Z-X. He and Z. Wang. Möbius energy of knots and unknots. Ann. of Math. 139 (1994) p.1-50.

[Fu] S. Fukuhara. Energy of a knot. A Fête of Topology. Y. Matsumoto, T. Mizutani, and S. Morita, eds, Academic Press (1987) p.443-452.

[Go-Ma] O. Gonzalez and J.H. Maddocks. Global curvature, thickness, and the ideal shapes of knots. Proc. National. Acad. Sci. USA. 96 (1999) p.4769-4773. 
[Go-Ma-Sm] 0. Gonzales, J.H. Maddocks and J. Smutny. Curves, circles and spheres. To appear. Physical Knots: Knotting, Linking, and Folding Geometric Objects in $\mathbb{R}^{3}$, AMS Special Session on Physical Knotting and Unknottin, Las Vegas, Nevada, April 21-22, 2001. J. A. Calvo, K. Millett, and E. Rawdon eds., Contemp. Math. 304, Amer. Math. Soc., Providence, RI (2002) p.187-194.

[He] Z.-X. He. On the Minimizers of the Möbius Cross Energy of Links, Experimental Math. 11 (2002) 243-248.

[Ku] G. Kuperberg. Quadrisecants of knots and links. J. of Knot theory and its ramifications 3 (1994) p.41-50.

[Kus] R. Kusner. On Thickness and Packing Density for Knots and Links, Contemporary Math. 304 (2002) 175-180.

[Ku-Su] R. Kusner, and J.M. Sullivan. Möbius energies for knots and links, surfaces and submanifolds. Geometric Topology (Proceedings of the 1993 Georgia International Topology Conference). AMS/IP Studies in Adv. Math., W. H. Kazez ed. Amer. Math. Soc. and International Press, Cambridge, MA. (1997) p.570-604.

[La] R. Langevin. Introduction to integral geometry. IMPA,Rio (1997) and CRM, Barcelona (1999) lecture notes.

[La-Mo] R. Langevin and G. Moniot. The modulus of a link. manuscript.

[La-Ni] R. Langevin and Y. Nikolayevsky. Three viewpoints on the integral geometry of foliations. Illinois J. of Math. 43 (1999) p.233-255.

[La-OH] R. Langevin and J. O'Hara. Conformal geometric viewpoints for knots and links I. Physical Knots: Knotting, Linking, and Folding Geometric Objects in $\mathbb{R}^{3}$, AMS Special Session on Physical Knotting and Unknottin, Las Vegas, Nevada, April 21-22, 2001. J. A. Calvo, K. Millett, and E. Rawdon eds., Contemp. Math. 304, Amer. Math. Soc., Providence, RI (2002) p.187-194.

[La-Ro] R. Langevin and H. Rosenberg. Fenchel type theorems for submanifolds of $S^{n}$. Comment. Math. Helv. 71 (1996) p.594-616.

[Lin] X.-S. Lin. Knot energies and knot invariants. J. Differential Geom. 44 (1996) p.74-95.

[Ma-Sm] J.H. Maddocks and J. Smutny. personal communication.

[Mi] J. Milnor. On total curvature of knots. Ann. of Math. 52 (1950) p.248-260.

[Na] N. Nakauchi. A remark on O'Hara's energy of knots. Proc. Amer. Math. Soc. 118 (1993) p.293-296.

[O’H1] J. O’Hara. Energy of a knot. Topology 30 (1991) p.241-247. 
[O’H2] J. O'Hara. Energy of a knot. Sugaku Expositions 13 (2000) p.73-90.

Updated version is available through web:

http://www.comp.metro-u.ac.jp/ ohara/texfiles/esurvey.dvi (or .ps)

[Po] H. Poincaré. Calcul des probabilités. Gauthier-Villars $2^{e ̀ m e}$ édition (1912).

[Sak] M. Sakuma. Problem no. 8 (in Japanese). The collection of problems on "Low dimensional topology and related matters", in Japanese, edited by S. Kojima and S. Negami (1987) p.7.

[San] L.A. Santalò. Integral geometry and geometric probability. Encyclopedia of mathematics and its applications Addison-Wesley (1976). 